\title{
The Macro Response Monte Carlo Method for Electron Transport
}

Michelle M. Svatos

(Ph.D Thesis)

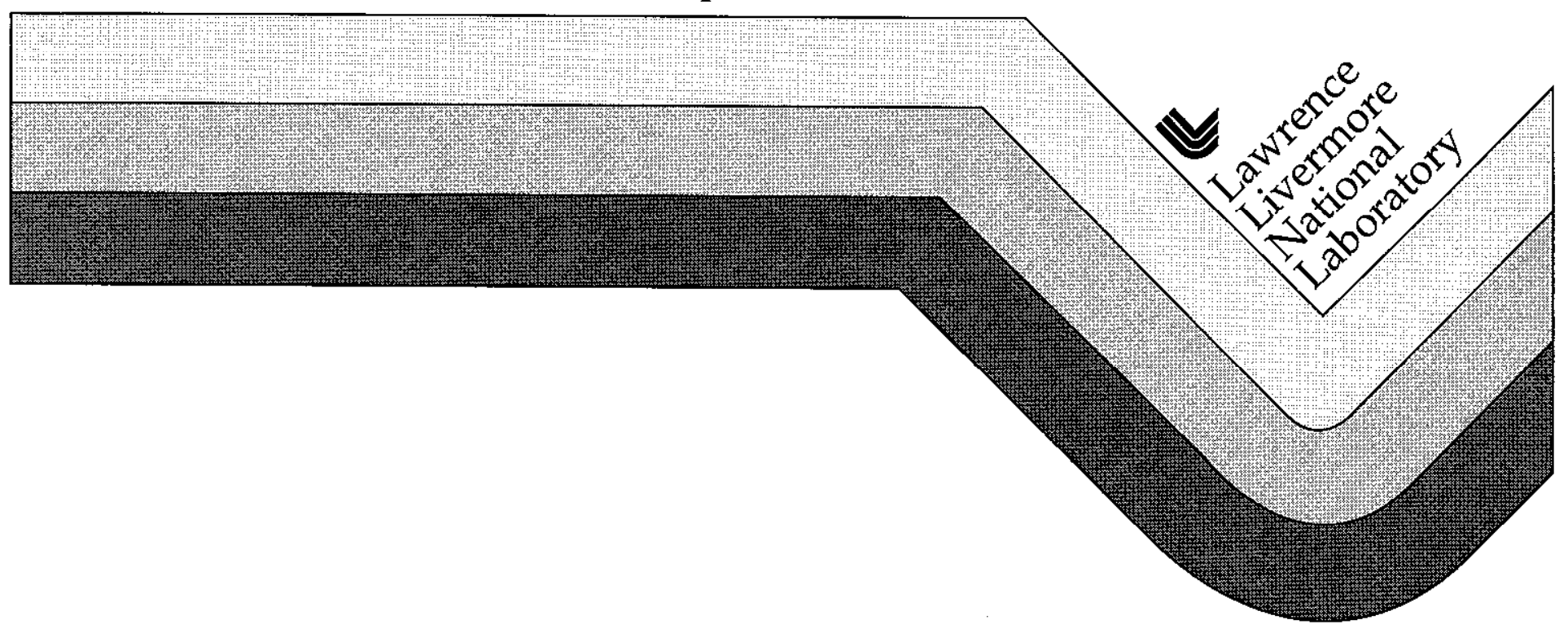




\section{DISCLAIMER}

This document was prepared as an account of work sponsored by an agency of the United States Government. Neither the United States Government nor the University of Califomia nor any of their employees, makes any warranty, express or implied, or assumes any legal liability or responsibility for the accuracy, completeness, or usefulness of any information, apparatus, product, or process disclosed, or represents that its use would notinfringe privately owned rights. Reference herein to any specific commercial product, process, or service by trade name, trademark, manufacturer, or otherwise, does not necessarily constitute or imply its endorsement, recommendation, or favoring by the United States Government or the University of California. The views and opinions of authors expressed herein do not necessarily state or reflect those of the United States Government or the University of California, and shall not be used for advertising or product endorsement purposes.

This report has been reproduced directly from the best available copy.

Available to DOE and DOE contractors from the Office of Scientific and Technical Information P.O. Box 62, Oak Ridge, TN 37831

Prices available from (615) 576-8401, FTS 626-8401

Available to the public from the National Technical Information Service

U.S. Department of Commerce 5285 Port Royal Rd., Springfield, VA 22161 


\title{
The Macro Response Monte Carlo Method for Electron Transport
}

\author{
Michelle M. Svatos \\ (Ph.D Thesis)
}

September 1998

LAWRENCE LIVERMORE NATIONAL LABORATORY

University of California $・$ Livermore, California $\bullet 94551$ 



\section{Abstract}

The main goal of this thesis was to prove the feasibility of basing electron depth dose calculations in a phantom on first-principles single scatter physics, in an amount of time that is equal to or better than current electron Monte Carlo methods.

The Macro Response Monte Carlo (MRMC) method achieves run times that are on the order of conventional electron transport methods such as condensed history, with the potential to be much faster. This is possible because MRMC is a Local-toGlobal method, meaning the problem is broken down into two separate transport calculations. The first stage is a local, in this case, single scatter calculation, which generates probability distribution functions (PDFs) to describe the electron's energy, position and trajectory after leaving the local geometry, a small sphere or "kugel".

A number of local kugel calculations were run for calcium and carbon, creating a library of kugel data sets over a range of incident energies $(0.25 \mathrm{MeV}-8 \mathrm{MeV})$ and sizes $(0.025 \mathrm{~cm}$ to $0.1 \mathrm{~cm}$ in radius).

The second transport stage is a global calculation, where steps that conform to the size of the kugels in the library are taken through the global geometry. For each step, the appropriate PDFs from the MRMC library are sampled to determine the electron's new energy, position and trajectory. The electron is immediately advanced to the end of the step and then chooses another kugel to sample, which continues until transport is completed.

The MRMC global stepping code was benchmarked as a series of subroutines inside of the Peregrine Monte Carlo code. It was compared to Peregrine's class II condensed history electron transport package, EGS4, and MCNP for depth dose in 
simple phantoms having density inhomogeneities. Since the kugels completed in the library were of relatively small size, the zoning of the phantoms was scaled down from a clinical size, so that the energy deposition algorithms for spreading dose across 5-10 zones per kugel could be tested. Most resulting depth dose calculations were within $2-3 \%$ of well-benchmarked codes, with one excursion to $4 \%$.

This thesis shows that the concept of using single scatter-based physics in clinical calculations would not only be possible, but would likely be more efficient than current methods, provided large kugels were generated.

This work was performed under the auspices of the U.S. Department of Energy by the Lawrence Livermore National Laboratory under contract no. W-7405-ENG48. 


\section{Acknowledgements}

The best thing about writing a thesis is having the opportunity to recognize some of the many people who made it possible.

At the top of my list is Rock Mackie, my official advisor on this project. In addition to conceiring of this type of electron transport many years ago, he arranged for me to continue my research at LLNL. Over the years, his creative energy and rich feel for the field as a whole have left a profound impression on me. The enthusiasm and sense of adventure within his group at the University of Wisconsin has resulted in a unique atmosphere that I have always especially enjoyed, even if tangentially.

Equal thanks goes to my on-site advisor at LLNL, Bill Chandler. I would still be bug hunting in my global code if it were not for his exceptional ability to track down bugs and the insightful test problems to which he suggested I subject my code. He not only did his best to keep me on track, he worked hard at keeping me in good spirits right until the end - at times a tough job.

I owe a very great debt to Jim Rathkopf, who served as my primary source of information on many things, including topics as diverse as alias sampling, statistical interpolation, collision physics, Monte Carlo speed-up techniques, gambling, U.S. history, football plays, Broadway musicals, child-rearing and brew pub locations; some of which were absolutely essential to the production of this work.

Paul DeLuca, the chairman of the Medical Physics Department at the University of Wisconsin, played a vital role as liaison between the UW and LLNL, as well as giving me a few excellent tidbits of technical advice.

Clint Ballinger and Hans Neuenschwander were, of course, the pioneers in the Local-to-Global transport area. Everything I have done here relied heavily on their 
previous implementations. I am very grateful for their eagerness and openness in sharing it with me.

Special acknowledgment goes to Alex Bielajew, for many technical discussions, criticisms, and inspirations.

Red Cullen and the late Ted Perkins provided an outstanding electron database, the EEDL, without which this work would not have been possible. They also provided many technical details in conversations and (in Ted's case) written memos which were invaluable.

I would like to thank Paul Bergstrom and Alexis Schach von Wittenau. In addition to serving as general sounding boards on a wide variety of topics, they ran the EGS4 and MCNP comparisons (respectively) that were essential for benchmarking my code. Paul Reckwerdt at the UW was always able to give me advice and ideas. I would also like to recognize the contributions of Marv Adams of Texas A and $M$, for helping me analyze some strange results that led to a crucial bug find; and Rosemary Walling of LLNL, for encouraging me to take this project "bird by bird".

While I am on a roll, I would like to reach back for a moment, and think about how I got started on this track. I remember Professor Sung Kyu Kim of the Macalester College Physics Department, who answered endless questions from a freshman in his Contemporary Concepts in Physics class. It was he who opened the door for me, and encouraged me - a somewhat unlikely candidate - to pursue physics. I hope that someday I can give something as valuable to someone else. I also thank Professor Rich Brundage, who supervised my research there, and Professor Ray Mikkelson, who agreed in a weak moment to teach me Quantum Mechanics as an independent study so I would be ready for graduate school.

Not to be neglected are the people who supported me in the other areas of my life during this long strange trip. Stanley Liu taught me ice hockey and ASL (America Sign Language), sometimes at the same time, which provided excellent 
relief from my computer. He also gets credit for introducing me to Mal May, who, in addition to arranging periodic puck-thumping therapy in her garage, has been an excellent listener, neighbor and overall inspiration. I must thank my team in the Oakland Ice Adult League, the Coyotes, who have always encouraged me to "go"; and perhaps more appropriately, my opponents, who occasionally bore the brunt of my frustrations.

I thank my parents, who always told me I could do anything. Hopefully by now they have forgiven me for taking that statement too literally at times during my years with them.

My deepest thanks goes to my husband, Erik Chell, who has taught me many great and subtle things. As a part of this, he has been giving me his unwavering support and encouragement, all while waiting patiently - for the last four yearsto take a vacation.... Let's go!! 


\section{Contents}

Abstract $\quad$ i

$\begin{array}{ll}\text { Acknowledgements } & \text { iii }\end{array}$

1 Electron Dose Calculation Methods 1

1.1 Introduction . . . . . . . . . . . . . . . 1

1.1.1 The Role of Electrons and their Simulation in Radiotherapy 1

1.1.2 Simulating the Transport of Electrons in Media . . . . . . 2

1.1.3 Approaches to Clinical Electron Dose Calculation . . . . . . 5

1.2 The "Right" Way: The Boltzmann Equation . . . . . . . . . 6

1.3 Deterministic Methods . . . . . . . . . . . . . . . . . . 8

1.4 Pencil Beam Transport: Fermi-Egyes Theory . . . . . . . . . . . . 9

1.5 The Monte Carlo Method . . . . . . . . . . . . . . . . 11

1.5.1 A Monte Carlo Method for Charged Particles: Condensed History . . . . . . . . . . . . . . . 13

1.5.2 Two Flavors of Condensed History: Class I and Class II . . 14

1.5.3 General Condensed History Assumptions . . . . . . . . 16

1.5.4 Strengths of Condensed History . . . . . . . . . . . 21

1.5.5 Condensed History Weaknesses . . . . . . . . . . . 21

1.5.6 Existing Condensed History Codes _. . . . . . . . 21

1.6 Local-to-Global Methods . . . . . . . . . . . . . . . . . . 26

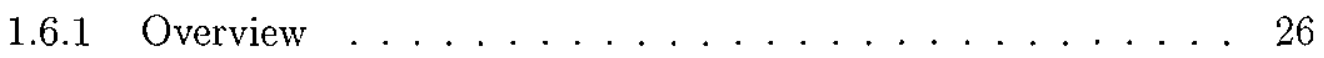

1.6.2 Response History Monte Carlo . . . . . . . . . . . . . 29

1.6 .3 Background . . . . . . . . . . . . . . . . 29

1.7 Comparison of the Monte Carlo Methods . . . . . . . . . . 32 
1.7 .1 Speed . . . . . . . . . . . . . . 32

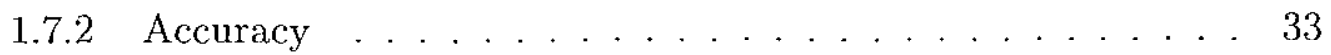

1.7 .3 Storage $\ldots \ldots \ldots \ldots \ldots \ldots \ldots$

1.7 .4 Summary . . . . . . . . . . . . . . . 34

1.8 Recent Developments . . . . . . . . . . . . . . . . 35

1.8.1 The OMEGA Project. . . . . . . . . . . 35

1.8.2 The Peregrine Project . . . . . . . . . . . . 37

1.9 Scope of this Work . . . . . . . . . . . . . . . 37

2 Single Scatter Electron Monte Carlo 39

2.1 Introduction . . . . . . . . . . . . . . . . . . . 39

2.1.1 Why Single Scatter? . . . . . . . . . . . . . . 39

2.1 .2 The EEDL Database . . . . . . . . . . . . . . 40

2.2 Physics Algorithms . . . . . . . . . . . . . . . . . . . 41

2.2.1 Simulating Individual Electron Interactions in Media . . . 41

2.2 .2 Compounds and Mixtures . . . . . . . . . . . . 57

2.3 CREEP Results . . . . . . . . . . . . . . . . . . 58

2.3.1 Backscatter Studies . . . . . . . . . . . . . . 58

2.3.2 Transmission Studies . . . . . . . . . . . . . 58

2.3.3 Energy Deposition Curves . . . . . . . . . . . 63

2.3.4 Other Quantities of Interest . . . . . . . . . . . . . 72

2.4 Discussion . . . . . . . . . . . . . . . . . . . . 72

2.5 Summary of Single Scatter . . . . . . . . . . . . . 73

3 The Macro Response Library $\quad 75$

3.1 Introduction . . . . . . . . . . . . . . . . . 75

3.2 Correlation of Phase Space by Exit Bands . . . . . . . . . . . 77

3.3 CREEP Kugel Phase Space Tallies . . . . . . . . . . . . . 78 
3.3.1 Primary Position Cosine . . . . . . . . . . . . . 80

3.3 .2 Primary Exit Energy . . . . . . . . . . . . . . . 8 80

3.3.3 Primary Exit Trajectory . . . . . . . . . . . . . . 81

3.3.4 Knock-on Exit Energy . . . . . . . . . . . . . . 8 86

3.3.5 Knock-on Position Cosine . . . . . . . . . . . . . 87

3.3.6 Knock-on Exit Trajectory . . . . . . . . . . . . 87

3.3.7 Photon Energy . . . . . . . . . . . . . . . . 87

3.3.8 Photon Position Cosine . . . . . . . . . . . . . . . . . 87

3.3.9 Photon Trajectory . . . . . . . . . . . . . . . 88

3.4 Other Tallies . . . . . . . . . . . . . . . . . 88

3.4 .1 Average Energy Expended . . . . . . . . . . . . 88

3.4.2 Band Probabilities and Boundaries . . . . . . . . . 88

3.4.3 Average Number of Knock-ons Escaping . . . . . . . . . . 91

3.4.4 Average Number of Photons Escaping . . . . . . . . . 91

3.5 Energies, Sizes, and Materials for the Library . . . . . . . . . . 91

3.5 .1 Energy Ladder . . . . . . . . . . . . . . . . . 92

3.5 .2 Sizes . . . . . . . . . . . . . . . . . . . 92

3.5 .3 Materials . . . . . . . . . . . . . . . 99 93

3.6 The Library Format $\ldots \ldots \ldots \ldots \ldots$

3.7 Storage Requirements for the Library . . . . . . . . . . . . . . 94

3.8 Library Generation and Navigation . . . . . . . . . 95

3.8.1 Serial Kugel Generation . . . . . . . . . . . . . 996

3.8.2 Nested Kugel Generation . . . . . . . . . . . . . . . 96

3.8.3 Bootstrapped Kugel Generation . . . . . . . . . . . . 97

4 Macro Response Transport $\quad 100$

4.1 Introduction . . . . . . . . . . . . . . . . . 100

4.2 Logic Flow . . . . . . . . . . . . . . . . . . . 100 
4.3 Determination of Step Size . . . . . . . . . . . . . . . 103

4.4 Determining the Kugel Energy . . . . . . . . . . . . . . . . 104

4.5 Determining the Exit Band . . . . . . . . . . . 105

4.6 Updating Phase Space After A Step . . . . . . . . . . . . . . 105

4.6.1 Determination of Exit Position . . . . . . . . . 105

4.6.2 Determination of Exit Trajectory . . . . . . . . . . . 107

4.7 Energy Deposition to the Transport Mesh . . . . . . . . . 110

4.7.1 Magnitude of Energy Deposition in Kugel . . . . . . . 110

4.7.2 Distribution of the Deposition Across Several Zones . . . . . 111

4.8 Boundary crossing . . . . . . . . . . . . . . . . . . 118

4.9 Tracking Secondary Particles . . . . . . . . . . . . . . . . 121

4.9.1 Treatment of Knock-on Electrons . . . . . . . . . . . 121

4.9.2 Treatment of Bremsstrahlung Photons . . . . . . . . 122

4.10 Interface with the Parent Tracking Code . . . . . . . . . . 122

$5 \quad$ Results of the MRMC Method 124

5.1 Introduction . . . . . . . . . . . . . . . . 124

5.2 Test Problem 1: Infinite Medium _ . . . . . . . . . . . 125

5.3 Test Problem 2: Single Density Inhomogeneity . . . . . . . . . 128

5.3 .1 Low Density . . . . . . . . . . . . . . . . . 129

$5.3 .2 \quad$ High Density . . . . . . . . . . . . . . . . 129

5.4 Test Problem 3: "Riblet" Phantom . . . . . . . . . . . . 131

5.5 Summary of Global Test Problem Results . . . . . . . . . . 134

5.6 Timing for Global Test Problems . . . . . . . . . . . . 136

6 Summary of the MRMC Method 138

6.1 Areas for Future Development . . . . . . . . . . . . 140

6.1 .1 Local Code Additions . . . . . . . . . . . . . . . 140 
6.1.2 MRMC Library Building and Extension . . . . . . . . . 141

6.1.3 Global Code Enhancements . . . . . . . . . . . . . . 143

6.2 Other Applications . . . . . . . . . . . . . . . . . . . . 144

A Further Details on Scattering Theories 146

A.1 Moliere theory of Multiple Scattering . . . . . . . . . . . . 146

A.2 Goudsmit-Saunderson Model for Multiscatter . . . . . . . . . 147

B Example Kugel PDFs

Bibliography 169 


\section{List of Tables}

1 A comparision of various features of electron transport in existing Monte Carlo codes . . . . . . . . . . . . . . . 34

2 Evaluated Electron Data Library contents for elastic scatter and ionization events. . . . . . . . . . . . . . . . . . . 42

3 Evaluated Electron Data Library contents for bremsstrahlung and excitation events. . . . . . . . . . . . . . . . 4 43

4 Timings for several CREEP runs in various media. . . . . . . . 73

5 The probabilities of a primary electron escaping from each surface band for calcium. . . . . . . . . . . . . . . . 89

6 The probabilities of a secondary "knock-on" electron escaping from each surface band for calcium. . . . . . . . . . . . . . . . . 89

7 The probabilities of a secondary bremsstrahlung photon escaping from each surface band for calcium. . . . . . . . . . . 90

8 Band probabilities for several kugels of varying size for carbon ( $\rho=$ $\left.2.0 \mathrm{~g} / \mathrm{cm}^{3}\right)$ at an incident energy of $6.5 \mathrm{MeV} \ldots \ldots . \ldots 90$

9 The size in bytes of the data files necessary for 1 kugel in uncompressed ASCII format. . . . . . . . . . . . . . . . . 94

10 Comparison of infinite calcium depth dose parameters for $4.9 \mathrm{MeV}$ electrons . . . . . . . . . . . . . . . . . 126

11 Comparison of low-density inhomogeneity calcium depth dose parameters for $4.9 \mathrm{MeV} \ldots \ldots \ldots \ldots$

12 Comparison of high-density inhomogeneity calcium depth dose parameters for $4.9 \mathrm{MeV} \ldots \ldots \ldots \ldots 131$ 
xii

13 Comparison of "riblet" calcium phantom depth dose parameters for

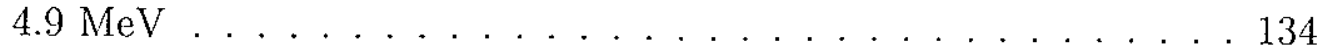




\section{List of Figures}

1 Example of a typical depth-dose curve for a electron beam. . . . . 3

2 Transport in the Macro Monte Carlo Method with "kugels". . . . . 29

3 Two separate portions of the problem of radiotherapy simulation. . 36

4 The logic flow of the CREEP analog electron scatter Monte Carlo code. . . . . . . . . . . . . . . . . . 4 45

5 An ionization interaction resulting in the liberation of an atomic electron. . . . . . . . . . . . . . . . . 47

6 An elastic scatter interaction. . . . . . . . . . . . . . 49

$7 \quad$ The effect of elastic scattering on a energy deposition versus depth

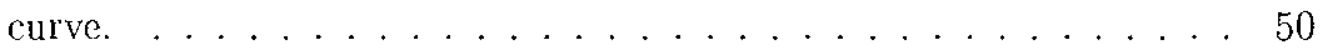

8 A electron giving rise to a bremsstrahlung photon in the field of a nucleus. . . . . . . . . . . . . . . . . 53

9 An incident electron causing excitation in an atomic electron. . . . 56

10 CREEP backscatter percentage compared to experimental results . 59

11 CREEP backscattered energy spectrum compared to experimental

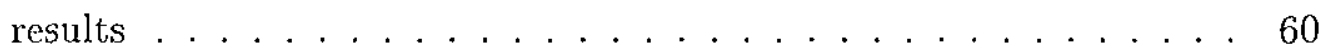

12 Transmitted energy loss spectra from gold slabs of two different thicknesses . . . . . . . . . . . . . . . . . . . 61

13 Transmitted energy loss spectra from a thin tantalum slab for two

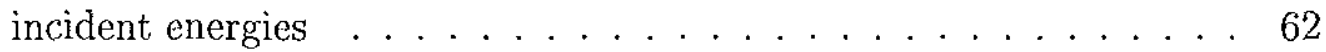

14 Energy deposition as a function of depth in aluminum for $300 \mathrm{keV}$

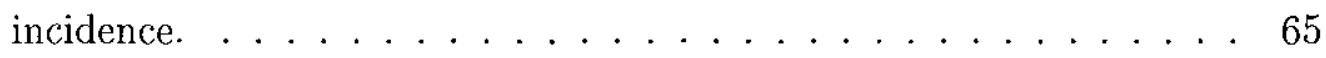

15 Energy deposition as a function of depth in molybdenum for $1 \mathrm{MeV}$

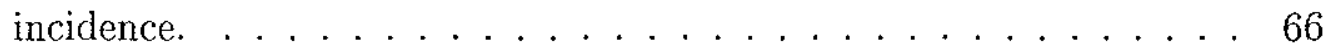


16 Energy deposition as a function of depth in tantalum for $500 \mathrm{keV}$ incidence. . . . . . . . . . . . . . . . . 6 67

17 Energy deposition as a function of depth in beryllium for 100, 300 and $500 \mathrm{keV}$ incidence. . . . . . . . . . . . . 68

18 Energy deposition as a function of depth in copper for $300 \mathrm{keV}$ and $500 \mathrm{keV}$ incidence. . . . . . . . . . . . . . . . . . . . 69

19 Energy deposition as a function of depth in iron for $300 \mathrm{keV}$ and 1 $\mathrm{MeV}$ incidence. . . . . . . . . . . . . . . 70

20 Energy deposition as a function of depth in carbon for $1 \mathrm{MeV}$ incidence 71

21 A partial schematic of the MRMC library. . . . . . . . . . 76

22 A diagram of the kugel geometry showing the four surface exit bands. . . . . . . . . . . . . . . . . . . 79

23 The local geometry for the tallying of the electron's exit trajectory. 83

24 The classical spherical coordinate system used to tally direction co-

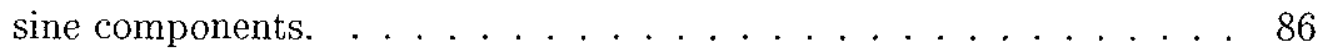

25 Three kugels in a concentrically nested configuration. . . . . . . 97

26 The "bootstrap" method generates a large kugel by using steps that are the size of a small kugel. . . . . . . . . . . . . . . . . 98

27 An overview of global transport through a CT mesh in MRMC. . . 101

28 The logic flow for Macro Response Monte Carlo transport. . . . . . 102

29 Geometry for the determination of the exit position $\ldots \ldots \ldots 106$

30 The global geometry for direction cosines . . . . . . . . . . 108

31 The difficulty with using the average energy deposition for the band 112

32 Two possible representations of the average electron path through the kugel for the purpose of energy deposition. . . . . . . . . . . 113

33 Step size artifacts associated with the simple radial path deposition 115

34 The three-segment path through the kugel $\ldots \ldots \ldots \ldots$ 
35 A comparison of paths through the kugel generated for $2.75 \mathrm{MeV}$ in calcium . . . . . . . . . . . . . . . 119

36 A comparison of paths through the kugel generated for $5 \mathrm{MeV}$ in

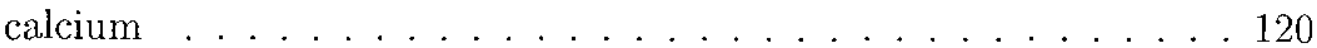

37 Illustration of the infinite medium phantom. . . . . . . . . . 126

38 Infinite calcium phantom depth dose comparison for $4.9 \mathrm{MeV}$ electrons. . . . . . . . . . . . . . . . 127

39 Illustration of the single-inhomogeneity phantom. . . . . . . . . . 128

40 Calcium phantom with a low-density inhomogeneity depth dose comparison for $4.9 \mathrm{MeV}$ electrons. . . . . . . . . . . . . . 130

41 Calcium phantom with a high-density inhomogeneity depth dose comparison for $4.9 \mathrm{MeV}$ clectrons. . . . . . . . . . . . . . . 132

42 Illustration of the "riblet" phantom. . . . . . . . . . . . 133

43 Calcium "riblet" phantom depth dose comparison for $4.9 \mathrm{MeV}$ elec-

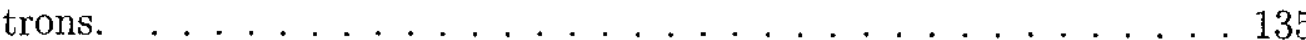

44 Exiting position cosines for primary electrons and escaping knockons from Band 1 of a $3 \mathrm{MeV}$ calcium kugel. . . . . . . . . . . . 150

45 Exiting position cosines for primary electrons and escaping knockons from Band 2 of a $3 \mathrm{MeV}$ calcium kugel. . . . . . . . . . . . . 151

46 Exiting position cosines for primary electrons and escaping knockons from Band 3 of a $3 \mathrm{MeV}$ calcium kugel.

47 Exiting position cosines for primary electrons and escaping knockons from Band 4 of a $3 \mathrm{MeV}$ calcium kugel. . . . . . . . . . . 153

48 Exiting energy for primary electrons and escaping knock-ons from Band 1 of a $3 \mathrm{MeV}$ calcium kugel. . . . . . . . . . . . . 154

49 Exiting energy for primary electrons and escaping knock-ons from Band 2 of a $3 \mathrm{MeV}$ calcium kugel. . . . . . . . . . . . . 155 
50 Exiting energy for primary electrons and escaping knock-ons from Band 3 of a $3 \mathrm{MeV}$ calcium kugel. . . . . . . . . . . . 156

51 Exiting energy for primary electrons and escaping knock-ons from Band 4 of a $3 \mathrm{MeV}$ calcium kugel. . . . . . . . . . . . . 157

52 Exiting trajectory (dcp) for primary electrons and escaping knockons from Band 1 of a $3 \mathrm{MeV}$ calcium kugel. . . . . . . . . . 158

53 Exiting trajectory (dcp) for primary electrons and escaping knockons from Band 2 of a $3 \mathrm{MeV}$ calcium kugel. . . . . . . . . . 159

54 Exiting trajectory (dcp) for primary electrons and escaping knockons from Band 3 of a $3 \mathrm{MeV}$ calcium kugel. . . . . . . . . . . 160

55 Exiting trajectory $(\mathrm{dcp})$ for primary electrons and escaping knockons from Band 4 of a $3 \mathrm{MeV}$ calcium kugel. . . . . . . . . . 161

56 Exiting trajectory $(\cos \mu)$ for primary electrons and escaping knockons from Band 1 of a $3 \mathrm{MeV}$ calcium kugel. . . . . . . . . . 162

57 Exiting trajectory $(\cos \mu)$ for primary electrons and escaping knockons from Band 2 of a $3 \mathrm{MeV}$ calcium kugel. . . . . . . . . . 163

58 Exiting trajectory $(\cos \mu)$ for primary electrons and escaping knockons from Band 3 of a $3 \mathrm{MeV}$ calcium kugel. . . . . . . . . . . 164

59 Exiting trajectory $(\cos \mu)$ for primary electrons and escaping knockons from Band 4 of a $3 \mathrm{MeV}$ calcium kugel. . . . . . . . . . . 165

60 Average number of escaping knock-ons from a $3 \mathrm{MeV}$ calcium kugel. 166

61 Average number of escaping bremsstrahlung photons from Bands 1 and 2 of a $3 \mathrm{MeV}$ calcium kugel. . . . . . . . . . 167

62 Average number of escaping bremsstrahlung photons from Bands 3 and 4 of a $3 \mathrm{MeV}$ calcium kugel. . . . . . . . . . . 168 


\section{Chapter 1}

\section{Electron Dose Calculation}

\section{Methods}

\subsection{Introduction}

\subsubsection{The Role of Electrons and their Simulation in Radio- therapy}

For all its relative success as a cancer treatment modality, radiotherapy still suffers from the fundamental need for more detailed knowledge about where the energy is being deposited in the patient. Traditionally absorbed dose computation has been based on correcting dose distributions that have been measured in a water phantom, as will be discussed below. It would be much more desirable to calculate the dose distribution from first principles. Although we presently have no means to calculate the exact dose distribution delivered in a particular patient, we are able to simulate the physics of a treatment in a patient's own anatomy, provided CT data are available for that patient. There are many methods of simulation, several of which will be discussed in this chapter.

Ultimately it is hoped that accurate simulation will lead to more conformal dose distributions, confidence in dosimetry, and optimization of treatment plans. The better the dose is conformed to the tumor, the higher the dose levels can 
be tolerated for the same complication rate; or conversely, the lower the complication rate can be for the same absorbed dose. Together these factors will bring about higher cure rates for tumors, and/or lower complication rates, allowing more patients to live comfortable lives.

Electrons play an important role in radiotherapy. Not only are they used as a primary beam in all or part of approximately $5-10 \%$ of all radiotherapy treatments, they also arise as secondary particles in all other treatments - most notably in those using photon beams, which account for the vast majority of all treatments.

The main reason primary electrons are used to treat certain tumors is that almost all of their energy is deposited up to a predictable depth in the medium, which means normal tissue downstream of the incident beam is spared. This can be seen in the typical electron depth-dose curve shown in Figure 1. The electrons will cause deposition of a very small amount of energy beyond their practical range in a medium, due to bremsstrahlung photons which they create as they (de)accelerate in the medium. These photons have a much lower probability of interaction than the electrons, and travel deeper as they exponentially attenuate. Electrons are often the modality of choice for tumors residing a few centimeters below the skin, especially when other critical organs are located beyond the tumor, such as in the head and neck region.

Electrons also have the advantage of being inexpensive to generate. In addition, they have been widely used for over thirty years, which has allowed much physician experience to be accumulated.

\subsubsection{Simulating the Transport of Electrons in Media}

Simulating electron interactions in media involves unique challenges relative to other types of particle transport for several reasons. First, the cross sections for electrons tend to be five or six orders of magnitude higher than those for neutrons 
Depth Dose for $10 \mathrm{MeV}$ e- in Water

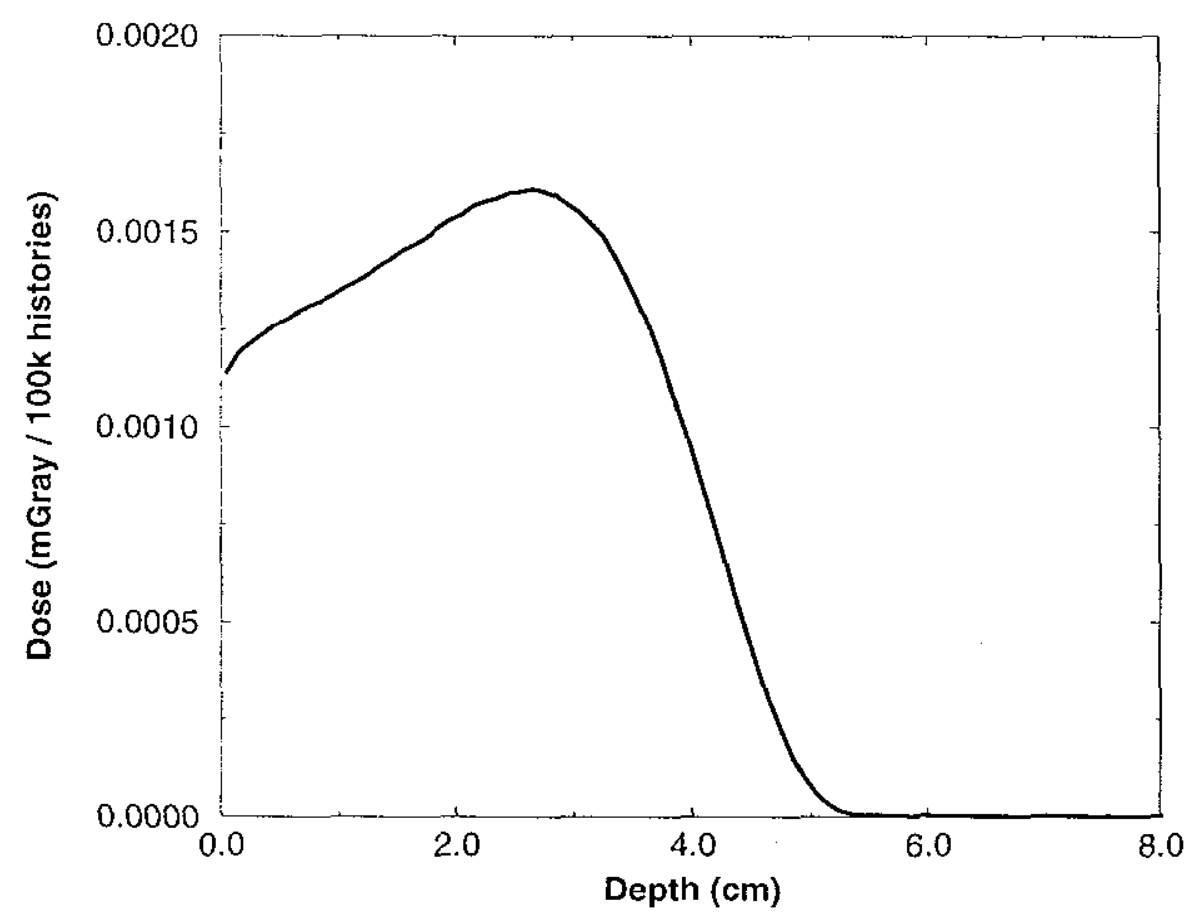

Figure 1: Example of a typical depth-dose curve for a electron beam. This is a 10 $\mathrm{MeV}$ electron beam normally incident on a water phantom. 
or photons of the same energy, so simulation of each collision has historically been too time-consuming to be done routinely. For instance, the mean free path of a 1 $\mathrm{MeV}$ electron in water is approximately $10^{-4} \mathrm{~cm}$, which is quite small in relation to the volumes of interest for most transport problems.

Secondly, the fractional energy loss per collision is frequently five orders of magnitude smaller for electrons than it is for photons. This indicates that the number of interactions required for an electron to give up all of its energy is very large. With a few rare but important exceptions, single interactions do not change the electron's energy or direction by a large amount. It is rather the cumulative effect of many small changes that result in the characteristics of the electron track. Thus it is important to have accurate physics for each event, since errors will easily compound as the electron slows down.

Finally, even though most of the changes in energy and angle are small, they are not insignificant. For heavier charged particles, one can usually make a simple (Gaussian-like) approximation for the lateral distribution; for electrons, these approximations do not hold as well because they tend to underestimate the degree of lateral transport.

The key to getting electron absorbed dose distributions correct in an inhomogeneous phantom or patient is to simulate the lateral transport correctly. To achieve this, it is crucial that the scatter angles are modeled accurately. In particular, the low-probability large-angle scatters and backscatters cannot be underestimated, for it is these events that lead to the complicated structure in the dose distributions, especially near media interfaces. 


\subsubsection{Approaches to Clinical Electron Dose Calculation}

Traditionally, electron dose distributions were approximated without doing any explicit electron transport at all, but rather by extrapolating from measured results taken for a particular energy, field size and source-to-surface distance (SSD) on a given accelerator. The extrapolation was done by modifying the absorbed dose that was experimentally determined under the reference parameters by ratios representing the change between those parameters and the parameters of interest. For example, if dose values were measured in water, a table of the percentage of the maximum dose as a function of depth (on-axis) in the water could be deduced. A similar table could be generated for off-axis ratios (OARs) by measuring lateral profiles at a variety of depths. The dose at a given point $P$ in the patient could then be estimated by knowing the maximum dose for the treatment and applying the percent depth dose (PDD) correction for the depth of $P$ in the patient, and an off-axis correction for the distance to $P$ from the axis at that depth.

Tables of PDDs were available for various sources (incident energies), field sizes, depths in phantom, and SSDs. To modify the dose for an SSD that was different from the reference SSD, the ratio of inverse distances squared would be applied. For a change in field size, tables of field-size-factors would be applied. To compensate for the curved surface of a patient, as opposed to the flat surface of the phantom, the isodose lines could be shifted along rays projecting from the source.

It is important to point out that these techniques are often quite successful for predicting dose in a homogeneous volume. In fact, in some cases they may be superior to some more advanced methods (for homogeneous volumes) because they are based on measurements from a particular machine; thus they circumvent the problem of how to model the electron distributions emerging from the source and incident on the patient/phantom, which can be a significant obstacle to transportbased methods. 
The real challenge for these empirical-based methods, then, lies in correcting the homogeneous water dose distribution to account for the many inhomogeneities present in the human body, such as air cavities, bone, dense muscle mass and sparse lung tissue.

One way of addressing this problem was to apply "equivalent-thickness corrections" to the water measurements. These corrections were based on compressing or stretching the isodose lines by factors that were derived from the relative electron density in the path through tissue as compared to that in water $[36,1]$. These methods, at their best, were useful for obtaining depth dose information in the center of a broad beam, for a medium consisting of homogeneous layers. Even then, they do not account for the fact that electrons interact not only with other electrons, but also with the nucleus. For example, the elastic scattering cross section, introduced in Chapter 2, depends on the atomic number of the material. The clinical implication of this is that not all materials behave like scaled-density water; real bone, for example, scatters much more than water of bone density.

\subsection{The "Right" Way: The Boltzmann Equation}

At the most fundamental level, radiation transport is an attempt to derive a distribution of the particles (in space, direction, time and energy) after they have traversed some distance into a medium. Formally, the way to begin this endeavor is by writing the general equation for transport from kinetic theory. Kinetic theory does not treat individual particles, but rather describes the aggregate behavior of continuous field of particles in a statistical manner [39].

Let $n$ be the number of particles that is expected within a differential volume element, $d^{3} \mathbf{r}$, with a velocity (which is directly related to its kinetic energy) that is within a differential velocity group, $d^{3} \mathbf{v}$, at time $t$. The function $n(\mathbf{r}, \mathbf{v}, \mathbf{t})$, called the "phase space density function", thus contains all of the information required 
for the description of standard transport processes; if a general solution is found for $n$, then the transport problem is solved.

Imagine that it is desired to transport the radiation through an arbitrary volume $V$, with a surface area $S$. The only mechanisms that can alter the number of particles in $V$ are flow through the surface of $V$, collision events within $V$, or sources of new particles located inside of $V$ :

$$
\left[\begin{array}{c}
\text { time rate } \\
\text { of change } \\
\text { of } \mathrm{n}
\end{array}\right]=\left[\begin{array}{c}
\text { change due } \\
\text { to flow } \\
\text { through } \mathrm{S}
\end{array}\right]+\left[\begin{array}{c}
\text { change due } \\
\text { to collisions } \\
\text { in } \mathrm{V}
\end{array}\right]+\left[\begin{array}{c}
\text { change due } \\
\text { to sources } \\
\text { in } \mathrm{V}
\end{array}\right]
$$

For the case of electron transport through tissue, the equation can be simplified due to a few basic facts about the nature of the problem. First, since we are not interested in information as a function of time (we only care about the total distribution after a treatment), we may consider a time-independent form of the above equation. Secondly, we can assume that the medium is in thermal equilibrium. This allows us to use the well-known Maxwell-Boltzmann equation to approximate equation 1.1. Thirdly, since the incident particles themselves do not interact with each other until fluences much higher than those used for radiotherapy are reached, terms higher than linear in the Boltzmann equation may be neglected. Thus the appropriate equation in differential form is

$$
\mathbf{v} \cdot \nabla n+v \Sigma_{t} n=\int v^{\prime} \Sigma_{s}\left(\mathbf{v}^{\prime} \rightarrow \mathbf{v}\right) n\left(\mathbf{r}, \mathbf{v}^{\prime}\right) d v^{\prime}+s
$$

where the first term reflects flow in and out of the volume; the second term accounts for the loss of the particles by the medium, where the third term represents the gain of particles from collisions, and where s represents sources from other nontransport processes. In this equation $\Sigma_{t}$ is the total interaction cross section which 
removes particles from the distribution and $\Sigma_{s}$ is the scatter cross section which results in a gain of particles to the distribution from collision events.

If a solution to the Boltzmann equation can be found, the problem of determining the dose distribution for that case is finished. All the methods below are different approaches to solving this equation.

\subsection{Deterministic Methods}

The Boltzmann equation (equation 1.2) can be made tenable computationally by using the technique of "differencing" [14]. This involves replacing the continuous operations with their corresponding discrete operations, such that the differentials become $\Delta$ 's and the integrals become summations. This idea could, in principle, be utilized in radiotherapy applications by writing (and differencing) a Boltzmann equation with appropriate three-dimensional boundary conditions for each CT voxel. However, a single CT scan will have on the order of 100,000 voxels in the dose grid. This vastly escalates the computational intensity.

There is a further problem with using this type of approach for electron transport in any setting. Numerical solutions for transport problems are best suited for smoothly varying functions in space and direction. However, charged particle transport problems, particularly those involving electrons, often require very anisotropic functions to describe their angular and spatial distributions. This means that a solution to equation 1.2 will require many high-order terms to have a satisfactory degree of accuracy ${ }^{1}$. This also causes a problem in computational intensity.

Nevertheless, numerical transport methods do have at least one potential advantage over other methods; namely, they can account for perturbations. If it

\footnotetext{
${ }^{1}$ It has been estimated by Cullen that as many as 800 Gauss-Legendre moments would be necessary to model the anisotropy for individual interactions [3]
} 
is not an absolute (accurate) answer that is desired, but rather knowledge about a trend in the output variable after a minor change in the input conditions, numerical methods can propagate the perturbation quickly. With the Monte Carlo method, in contrast, the entire simulation would have to be run again with the new input specifications, and would be subject to statistical noise which may hide the perturbation.

\subsection{Pencil Beam Transport: Fermi-Egyes The- ory}

The Pencil Beam method sums the dose distribution from individual small diameter rays called pencil beams. A pencil beam is made up of particles which pass through a differential cross sectional area, $\delta x \delta y$. The off-axis dependence of the dose distribution for each pencil beam is described by the Fermi-Eyges theory of thick-target multiple Coulomb scattering [23]. The on-axis dose information is obtained from measured depth-dose data.

The calculation assumes that the patient or phantom can be represented by a stack of slabs of different material types. Each individual slab is homogeneous and infinite in lateral extent. Assuming a single pencil beam has normal incidence on such geometry, the dose at a position $(\mathrm{x}, \mathrm{y}, \mathrm{z})$ can be expressed as

$$
D(x, y, z)=f(x, y, z) g(z)
$$

where the first factor is the off-axis term and the latter factor is the central-axis term. According to Fermi-Eyges theory, the off-axis term may be represented by a Gaussian of the form 


$$
f(x, y, z)=\frac{1}{2 \pi \sigma^{2}} \exp \left(-\frac{x^{2}+y^{2}}{2 \sigma^{2}}\right)
$$

where $\sigma$ is the angular spread about the axis including the penumbra. The on-axis term may be expressed in terms of its relationship to the depth dose in water, $g_{0}$

$$
g(z)=g_{0}\left(z_{e f f}\right)\left[\frac{\mathrm{SSD}+\mathrm{z}_{\mathrm{eff}}}{\mathrm{SSD}+\mathrm{z}}\right]^{2}
$$

where SSD is the source-to-surface distance, and $z_{\text {eff }}$ is the effective depth in water, assuming the linear stopping power of the material at depth $z$ is relatively independent of electron encrgy for normal body tissue.

The quantity $g_{0}$ can be related directly to a depth dose curve measured in a water phantom, assuming a uniform incident beam, by the equation

$$
D_{0}(x, y, z)=\frac{g_{0}(z)}{2 \pi \sigma_{0}^{2}} \int_{x} \int_{y} \exp \left(-\frac{\left(x-x^{\prime}\right)^{2}+\left(y-y^{\prime}\right)^{2}}{2 \sigma_{0}^{2}}\right) q d x^{\prime} d y^{\prime}
$$

where the limits of the integral depend on the extent of the field at depth $z$.

One of the most widely used simulation methods in clinical electron radiotherapy is an application of Fermi-Eyges theory in the so-called Hogstrom method [32]. The strength of the Hogstrom method of simulation is that it is fast enough for clinical use, on the order of minutes. It also uses measured data for input that may be specific to the treatment unit. It models penumbra in homogeneous situations (e.g., water phantoms) reasonably well, generally within approximately $10 \%$. It also models the dose distribution downstream from inhomogeneities that can be considered "infinite slabs" in the lateral plane with the same success.

Aside from the geometrical assumptions of infinite homogeneous slabs, the primary weakness of this method is that it ignores the production of secondaries, and 
the energy loss that occurs from them. In reality, the Moller (electron-electron) scattering changes the shape of the scatter distribution. The other major shortcoming of the Hogstrom method, and Fermi-Eyges theory in general, is that it does not model single large angle scattering events, including backscatter. Neglecting backscatter results in an underestimation of dose near bone or other high atomic number interfaces.

The Hogstrom method has been found to under-represent hot and cold extremes in energy deposition by approximately $10 \%$ [32]. Although the ability to run simulations quickly allows clinicians to try several different treatment plans, errors of this magnitude are bound to have clinical significance in some cases.

\subsection{The Monte Carlo Method}

Monte Carlo is a general problem solving tool which has seen applications in many diverse fields including mathematics, economics, traffic control, and radiation transport. The concept can be summarized by imagining the Monte Carlo code as a black box. In each case, there are four required inputs to the box; namely, a random number generator, the source characteristics, simulation geometry, and a probability distribution governing the possible outputs.

The output of the box uses the random number to index the probability distribution in some way, and a corresponding physical or mathematical result in generated. Usually this process is repeated many times, such that the result from each iteration is combined with all previous results until the desired statistical accuracy for the solution is achieved.

In electron transport problems, the Monte Carlo method is an indirect numerical solution to the Boltzmann equation, based on statistical samples. The technique consists of sampling the probability distributions (which contain the 
aggregate behavior of the Boltzmann equation) to govern the individual interactions of electrons in a given material. Simulated transport proceeds by moving sequentially from one physically valid set of electron variables (energy, location, and direction) to the next. The code keeps track of the physical quantities of interest (i.e., energy deposited) for a large number of histories to provide the required information about average quantities at the end of the simulation. The higher the number of histories, the smaller the sampling error, and the closer the approximation is to a continuous, Boltzmann solution.

Monte Carlo methods simulate electron transport by attempting to model the effect of the physical interactions taking place. They are capable of accurately modeling the dose response in a variety of difficult geometries, including anthropomorphic phantoms $[18,55]$. There are several categories of Monte Carlo algorithms commonly used for electron transport, which will be introduced in this section.

The main limitation of the Monte Carlo method applied to radiotherapy is the run time required to obtain reasonable statistical accuracy in the dose. Typically, running times for good statistics on a commonly available workstation would be measured in hours or tens of hours ${ }^{2}$, whereas a desirable time frame for a clinical code would be approximately ten minutes or less. However, this limitation will perhaps be overcome with the advent of a Monte Carlo code written especially for tracking photons and electrons through a regular Cartesian CT scan, as will be introduced in Section 1.8.2.

There are three main ways Monte Carlo can be sped up to approach the desired time window [39]. First, better computer hardware can (and will) be built, which should allow traditional electron transport codes (for example, condensed history codes, which will be introduced in Section 1.5.1.) to be fast enough for clinical use in three to eight years ${ }^{3}$. Secondly, a variety of statistical shortcuts such as

\footnotetext{
${ }^{2}$ The times are quite dependent on the complexity of the geometry and the size of the field.

${ }^{3}$ extrapolating from developments over the past ten or so years
} 
variance reduction, roulette, range reduction, and correlated sampling have been employed with some success [11]. These methods combined can reduce computing time significantly, although the exact amount is highly problem-dependent [12]. The main drawback to these techniques is that they must be applied with great caution to avoid biasing the physics in ways that change the answer significantly in regions of interest.

Finally, Monte Carlo can be sped up by the development of new and faster algorithms. For example, one success in this area is the Macro Monte Carlo (MMC) code, introduced in Section 1.6.1, which is able to simulate electron transport through a CT-volume in 200 seconds. ${ }^{4}$

It is in this category that this research attempts to make a contribution with the Macro Response Monte Carlo code.

\subsubsection{A Monte Carlo Method for Charged Particles: Con- densed History}

In the previous section it was found that analog techniques for charged particle transport suffer from computational difficulties due to the large number of collisions that must be modeled. Condensed history is a technique which circumvents this problem by combining many collisions into a single "step" or "jump" through the medium. Rather than attempting to model all interactions, condensed history approximates the aggregate effect of multiple collisions after a given step size. The resulting distributions are somewhat more isotropic and much easier to model.

In order to simulate the phase space of an electron after it has had many collisions, distributions which approximate their net effect on energy and angle are made. These distributions are sampled at the end of each step. The step size may

\footnotetext{
${ }^{4}$ The problem was a monoenergetic, parallel $10 \mathrm{MeV}, 5 \mathrm{~cm} \times 5 \mathrm{~cm}$ electron beam on a head phantom, with a resolution of $0.25 \mathrm{~cm} \times 0.25 \mathrm{~cm} \times 1 \mathrm{~cm}$. A million incident electron histories were calculated on a $200 \mathrm{MHz}$ Pentium Pro running Linux 1.2.13.
} 
be thought of in terms of the physical distance traveled or the energy difference between consecutive steps as the electron slows down. It must be emphasized that a condensed history step is not a physical reality for either the incident particle or the medium; it is simply a convenient (but artifactual) discretization that is used to periodically update the state of the particle.

There are several categories of approximations about the particle's interactions that are made in order for this technique to work. It will be seen that these approximations, which are discussed below, become less accurate when the energy region of interest is of the same order of magnitude as the atomic energy states; i.e., in situations such as low kinetic incident energy (below $100 \mathrm{keV}$ ), and high atomic number materials. Since condensed history algorithms are designed to run efficiently, it is desirable that they have as few steps as possible, because the run time for the code will be proportional to the number of steps. They also rely on multi-scatter distributions (discussed below) which require a large number of individual interactions occurring within each step to be valid. However, other assumptions, such as the assumption that the energy can be deposited along a straight line representing the path, become worse as the step size increases [9].

\subsubsection{Two Flavors of Condensed History: Class I and Class II}

Condensed History code algorithms were first divided into two categories, broadly known as Class I and Class II, by Berger [5]. This convention has been adopted and discussed by many authors since then.

In Class I condensed history algorithms no individual interactions are modeled, and all energy loss is assumed to be some constant amount per unit distance. The Class I schemes group all interactions together in each step and summarize the total effect at the end of the step by random sampling. The step sizes are 
predetermined, being either constant or logarithmically spaced. They use multiscattering distributions exclusively.

Class II schemes, on the other hand, use restricted multi-scattering distributions for the majority of the events, but model certain "catastrophic" events individually, such as bremsstrahlung and ionizations giving rise to secondary electrons ("knock-ons" or "delta-rays") having an energy greater than some predetermined cutoff value, $T_{\Delta}$. A "catastrophic" mean free path is determined $\left(\lambda_{\text {cat }}\right)$ from the sum of the cross-sections of the events which are considered catastrophic. The step length is given by

$$
s=-\lambda_{\text {cat }} \ln (1-\xi)=-\lambda_{\text {cat }} \ln (\xi)
$$

where $\xi$ is a uniformly distributed random number on the interval $(0,1)$. Noncatastrophic events are assumed to occur continuously throughout the pathlength, causing a uniform mean energy loss per unit distance given by the restricted stopping power for the medium.

Each scheme has advantages. Class II algorithms model the initial state of bremsstrahlung photons and knock-on electrons explicitly, which is a closer approximation of the true physical process. It is also the first step towards allowing their histories to be explicitly and independently followed. This allows their angular deviations to be more accurate and the correlation between energy loss and angular deflection is always conserved [2]. Class II algorithms are dependent on the threshold energy for knock-on electrons, $T_{\Delta}$. The Class I algorithms are inherently free of this dependency, but they are not able to model large-energy-loss events as accurately since they don't account for knock-on electrons and photons carrying energy away from the primary track. 


\subsubsection{General Condensed History Assumptions}

1. Straight-Line Approximation

In condensed history codes, the average distance traveled is calculated from the CSDA (Continuously Slowing Down Approximation) range. The electron path over that distance is assumed to be a straight line segment, whereas in reality, the electron is being deflected slightly after each interaction. The larger the step size, the worse this approximation becomes. The approximation is also worse in high atomic number $(\mathrm{Z})$ materials since the electrons scatter at larger angles per collision than for low Z materials.

\section{Multiple-Scatter Probability Distributions}

As mentioned previously, condensed history algorithms must assume a distribution of possible scatter angles at the end of each step. Probability distributions representing the spread of net angular deflections after a step are typically based on theories by either Moliere [8] or Goudsmit and Saunderson [24, 25].

- Moliere Model for Multiscatter

The Moliere approximation is valid for electrons that have undergone many small deflections ( $>20$ scatters). The derivation relies on the use of the small angle approximation, $\sin \theta \approx \theta$, which limits the validity of the theory to multiple scattering angles, $\theta$, which are less than approximately 20 degrees. More details of the Moliere distribution can be found in Appendix Section A.1.

- Goudsmit-Saunderson Model for Multiscatter The Goudsmit-Saunderson scattering theory is more robust than that of Moliere. It does not rely on a small angle approximation, and it doesn't 
explicitly require a large number of individual scattering events. Multiangle scatter distributions are constructed simply by estimating the average scattering angle for each collision and the number of collisions that have occurred in a step [25].

Although the Goudsmit-Saunderson model does not explicitly require a fixed number of scatter events per step in order to be valid, it does have some computational limits. As can be seen in Appendix Section A.2, the distribution takes the form of a Legendre series. As the pathlength becomes shorter (which makes the straight-line approximation better), higher orders are required in the Legendre series to make the expression accurate, especially at large angles. Berger and Wang [7] report as many as 999 terms may be required if the step size is reduced to 20 mean free paths in carbon and gold.

- Comparison of the Two Multi-Scatter Models

Both the multi-scatter distributions perform equally well for small scattering angles at relatively high energies and low atomic number materials, which probably constitute the majority of cases in radiotherapy. Beyond those conditions, Moliere distribution and the GoudsmitSanderson distributions each have their respective niches [7]. The Moliere distribution has the advantage that is relatively quick and easy to code (and sample) because it is a universal function of a scaled variable. This is makes it very convenient for finding the multi-scatter angle given a randomly selected pathlength. The disadvantages of using a Moliere distribution at low energies stem from the small angle approximation (which limit its use to deflections less than about 20 degrees) and the competing need for many scatter events in each step. ${ }^{5}$ At high energies,

\footnotetext{
${ }^{5}$ Bielajew [9] has found a way to overcome this limitation, see Section 1.5.6
} 
problems may arise because the Moliere theory does not take quantum spin and relativistic effects into account.

The Goudsmit-Saunderson distribution, on the other hand, is more general and robust. It is applicable for much smaller pathlengths than Moliere and it is valid for any scattering angle. It can be used with any elastic-scattering cross section calculated with any potential, including those with spin and relativistic effects accounted for. It is not difficult to correctly differentiate between the scattering of electrons and positrons. The real problem with the Goudsmit-Saunderson distribution is its complexity, which results in tedious coding and slow execution. This can be somewhat ameliorated by storing pre-calculated distributions for a chosen set of pathlengths, and sampling them at run time, but the code then relics on non-random pathlengths.

3. Uniform Energy Loss Across a Step (dE/dx)

Since condensed history methods do not consider the amount of energy lost to the medium for each individual interaction, they must have an alternative method of distributing the energy loss. As mentioned in section 1.5.2, Class I transport assigns an amount of energy loss directly proportional to the length of the (straight) path the particle took during the step. The amount is simply the average amount an electron of a particular energy gives up to the medium, which is varied slightly. Class II transport does the same for non-catastrophic energy losses.

The stopping power (or $\mathrm{dE} / \mathrm{dx}$ ) is the average amount of energy lost to the medium per unit distance. Most of this energy lost to the medium is transferred via ionization and excitation of atomic electrons. The extent of this energy loss, in $\mathrm{MeV} \mathrm{cm}^{2} / \mathrm{g}$ for an electron can be calculated by Bethe's expression [8] modified by Rorlich and Carlson: 


$$
\left(\frac{S}{\rho}\right)_{i o n}=\frac{2 \pi N_{A} r_{0}^{2} m_{0} c^{2}}{\beta^{2}} \frac{Z}{A}\left[\ln \frac{\tau^{2}(\tau+2)}{2\left(I / m_{0} c^{2}\right)^{2}}+\frac{\tau^{2} / 8-(2 \tau+1) \ln 2}{(\tau+1)^{2}}+1-\beta^{2}-\delta\right],
$$

where $\tau$ is the kinetic energy of the electron in $m_{0} c^{2}$ units; $\beta$ is the ratio $\mathrm{v} / \mathrm{c}$; $N_{A}$ is Avagadro's number, $6.022 \times 10^{23}$ per mol; $I$ is the mean ionization potential in $\mathrm{MeV}$; and $\delta$ is the density effect correction factor. Note this equation shows the stopping power is dependent upon the medium through three parameters: Z/A, I, and $\delta$. If a stopping power is used for energy loss, some compensation for the uniformity of the energy loss is required, as will be discussed in the next section.

Equation 1.8 has been previously calculated for a wide variety of materials and energies, and the resulting tables of stopping powers are available from agencies such as the International Commission on Radiation Units and Measurements (ICRU) [47].

\section{The Need for Energy Straggling Across a Step}

Suppose a charged particle of energy $T_{0}$ is incident on a layer of material. When it reaches the other side of this layer, the particle will have an energy $T_{1}$ which is less than its initial energy. Now suppose a series of identical particles are allowed to traverse this layer of material. The energy of the particles on the other side will not be constant, but rather will follow a distribution of energies with considerable fluctuation. This is known as energy "straggling". An expression for the energy loss distribution was derived by Landau [35]. He expressed the probability of an energy loss between $\Delta$ and $\Delta+d \Delta$ as 


$$
f_{L}(\Delta, s) d \Delta=\phi_{L}(\lambda) d \lambda
$$

where $s$ is the pathlength and $\phi_{L}(\lambda)$ is a universal function of a scaled energy loss parameter $\lambda$, which is available in tabular form. The relation between the scaled variable and the actual energy loss is given by

$$
\Delta=\xi\left\{\lambda+\ln \left[\frac{2 \xi m c^{2} \beta^{2}}{\left(1-\beta^{2}\right) I^{2}}\right]-\beta^{2}+0.4228-\delta\right\},
$$

where

$$
\xi=\frac{0.154}{\beta^{2}} \frac{Z}{A} s
$$

The mean energy loss of the Landau distribution is the stopping power for a particular material and energy. The stopping power method of handling energy loss across a step is a mean energy loss per unit distance; the "true" energy loss that would have occurred over such a step for a given electron is some variation about the mean value. Because the Landau distribution may be a relatively expensive method of treating energy loss, the effect is often approximated by using a stopping power spread out by a Gaussian distribution. There are various methods for estimating the width of such a Gaussian; Blunck and Westphal [10] recommend

$$
\sigma^{2}=Q Z^{\frac{4}{3}} \bar{\Delta}
$$

where $\bar{\Delta}$ is the mean energy loss in the pathlength $s$, and $Q=10 \mathrm{eV}$. 
There are several points to consider concerning the use of energy straggling corrections. First, the corrections are better when the step size is relatively small, however, we have scen that in small-angle scattering theories, the step size must be large enough to include enough collisions for the approximations to be valid. Secondly, most of the straggling is due to knock-on electrons of appreciable energy. If a Class II algorithm is used, these will be tracked as independent histories if they are above the threshold energy. However, if a method could explicitly account for the distribution pattern due to lower energy secondaries, there would be no need to induce a widening of an average energy loss.

\subsubsection{Strengths of Condensed History}

Numerous condensed history codes have been shown to have good agreement with experiment for high kinetic energies and in homogeneous materials. They are also quite efficient, with run-times only slightly longer than the times required for an analog photon Monte Carlo code running in a similar volume.

\subsubsection{Condensed History Weaknesses}

As was mentioned above, each of the fundamental assumptions upon which the condensed history algorithm rests begin to fail when the medium is a high atomic number material, when the kinetic energy of the primary particle becomes low, and/or when the particle encounters inhomogeneities in the medium [4].

\subsubsection{Existing Condensed History Codes}

This section contains a brief introduction to a few codes that are presently available in the scientific community for doing Monte Carlo transport of electrons (as well as some neutral particles). Although this is by no means an exhaustive list, these 
are codes that are reliably benchmarked and whose results are generally accepted. They are presented from the point of vicw of their suitability for use in radiotherapy simulation.

\section{ETRAN}

The ETRAN (Electron Transport) code was released in 1970 by Berger and Seltzer [6]. It uses Class I electron transport to treat both high and low-energy processes (nominally up to $1 \mathrm{GeV}$ and down to $1 \mathrm{keV}$ ), accounting for fluorescence, the effect of atomic binding on atomic electrons, and energy-loss straggling. ETRAN makes use of Goudsmit-Saunderson multiple angular scatter distributions, which avoid the small-angle approximations intrinsic to Moliere. It uses a sampling of a Landau distribution for energy loss [26]. In spite of its excellent accuracy for its time and availability, ETRAN went largely unnoticed for several years. A later version of this code was released under the name Sandyl [15].

\section{MCNP}

The MCNP (Monte Carlo N-Particle) Code was developed at Los Alamos National Laboratory as a three-dimensional, time-independent, general purpose transport code [13]. It was released for public use and found application primarily in nuclear power plants and shielding problems. In the late 1980's, electrons were added to the code, in a Class-I (ETRAN-like) package which gives the net flux through a zone or voxel. Since it was designed to work for many different applications, the Fortran-77 code has many options (such as variance reduction schemes) which leads to a code which is very large and bulky. Many arrays in the code are zonedependent; the code is suited best to geometries divided into $200-500$ regions. (CT scans have on the order of 100,000 voxels.) The run times for typical radiotherapy problems for standard MCNP4A would likely be on the order of several hours, 
which is too long to be acceptable for most routine clinical applications.

More recently, however, MCNP has been used to model the dose distributions in boron-neutron capture therapy $[27,28,29,50,37]$, which is an experimental treatment using beams of epithermal neutrons. There have also been serious efforts to evaluate its use as a tool for modelling general photon and electron radiotherapy treatment planning $[31,33]$. Modifcations in photon transport for lattice geometry, as well as algorithm development for dose tallies have been undertaken by a group at the University of California Los Angeles [20]. A preprocessor called RTMCNP (Radiation Therapy MCNP) is also under development there, which will provide a convenient interface into the MCNP4A command structure for radiotherapy treatment planning calculations [20]. Since the run times are still too long for routine use, this system is not presently intended to replace conventional means of treatment planning. However, it could serve as an analysis tool for some cases, and as a rhobust tool for further general algorithm development.

\section{EGS4}

History The Electron-Gamma Shower (EGS) code is a self-proclaimed "child of a thousand mothers and fathers" [41]. It originally evolved out of a Fortran code called SHOWER written in 1963 by Nagel for high energy electrons $(<1000$ $\mathrm{MeV}$ ) incident upon lead in cylindrical geometry. The database was later extended to include any element and a number of compounds. In the early 1970s, it was further extended to include photon interactions by a group at Stanford University, and renamed EGS. At this time it was also translated from Fortran to an obscure and since-dead ${ }^{6}$ - language called Mortran. The geometry was generalized through the use of a user-written routine. EGS3 was released in 1978 and soon became the industry standard for simulating electromagnetic cascades in various geometries

\footnotetext{
${ }^{6}$ It may be considered dead because its creator died and took most of its secrets with him; however, in the Latin sense of a non-evolving language, it is still widely used.
} 
at energies up to a few thousand $\mathrm{MeV}$ down to kinetic energies for electrons of 1 $\mathrm{MeV}$. The code was again considerably modified by Rogers and Bielajew in the early 1980s for energies as low as $10 \mathrm{keV}$ (EGS4), making it suitable for medical applications.

Transport Physics EGS4 uses Class II electron transport, with a stopping power with straggling for energy loss and with Moliere small angle scattering. The Moliere theory has been recently extended by Bielajew to free it from the need for $>20$ scattering events per step. In fact, Bielajew has made it possible to use a version of Moliere to simulate single scattering [9]. In general, the condensed history steps are of variable size. An algorithm called PRESTA chooses large steps when the distance to the next boundary is large, and takes smaller steps as the boundary is approached, for better resolution of interfaces. It also uses a path length correction which takes into account the difference between the straight line approximation and the curved pathlength for each electron step, as well as a lateral correction at the end of a step.

The following physics processes are taken into account by default [42] in EGS4:

- Bremsstrahlung production, using the Schiff formula for angular distribution

- Positron annihilation in flight and at rest

- Moliere multiple and singular scattering

- Moller (e-e-) and Bhabha (e+e-) scattering

- Continuous energy loss between discrete, catastrophic, interactions

- Total stopping power consists of soft bremsstrahlung and collisional loss terms

- Collision loss determined by the (restricted) Bethe-Bloch stopping power with the Sternheimer treatment of the density effect. 
- Pair production

- Compton scattering with binding effect correction

- Coherent (Rayleigh) scattering included by means of an option.

- Photoelectric effect (without fluorescent photons or Auger electrons)

In addition, EGS4 provides many options, including

- Electromagnetic Field transport

- $\mathrm{K}$ and L-shell fluorescence

- Ability to "force" photon interactions

- Variance reduction techniques: splitting and range rejection

Application to Radiotherapy EGS4 tends to be used indirectly for radiotherapy applications, such as its use to generate "kernels" in the convolution method, "kugels" in the MMC method (which will be introduced in Section 1.6.1), and to model detectors. One of the most significant drawbacks of using EGS4 in a clinical setting, in addition to its bulky size, is that it is still relatively difficult to use. Users must learn Mortran to write two subroutines, for scoring and representing the simulation geometry. Using the code also relies on the use of Mortran macros to change aspects of the internal code, which is a complicated venture that is prone to error. Even so, EGS4 is used regularly by a community of an estimated 6000 scientists [41]. It is an extremely well benchmarked code, and any code that hopes to establish itself in this area will certainly need to meet its performance standards before gaining acceptability. 


\subsection{Local-to-Global Methods}

As the following section will attempt to demonstrate, in order to meet the demands of speed and accuracy for this application, it is possible to make the sacrifice in another - hopefully more attainable - arena: storage. This scheme is especially attractive given the fact that the application has been defined and requires only a relatively narrow subset of energy ranges and materials.

\subsubsection{Overview}

Recently another class of charged particle transport methods has evolved which could generally be classified as "Local-to-Global Monte Carlo" transport. They are based on the use of pre-calculated probability distributions representing the change in phase space a charged particle would have after exiting a given volume element. The probability distribution functions (PDFs) for an exit state, given a particular incident state, would be calculated by traditional, high-statistic runs of another Monte Carlo code. The process of generating these PDFs is referred to as the "local calculation" and the geometry over which the calculation is computed is the "local geometry". The Local-to-Global Monte Carlo transport proceeds by advancing the particles through the medium during the global calculation in steps constrained to the size and shape of the local geometry. For each step, the incident phase space on the local geometry is the exit phase space of the previous step, until the particle is either below the cut-off energy or has escaped the global geometry. (In radiotherapy treatment planning problems, the global geometry is defined by the patient CT data.) This process requires a library of PDFs for a variety of incident energies and materials, each generated from a separate local calculation. 


\section{Macro Monte Carlo (MMC)}

The concept of Local-to-Global Monte Carlo was first proposed in the literature by Mackie and Battista [40] in 1984. In order to speed up electron transport, they proposed an algorithm called "Macro Monte Carlo" which would use cubical voxels as the local geometry. The incident state in phase space of an electron would be known, then it would "travel" through the cube as if the cube were a "black box" and the output state would immediately be sampled from a distribution of possible output states. These distributions would have been previously determined by a condensed history Monte Carlo code on a cubical geometry. The transport would continue by placing each cube end to end, depositing energy along the way. The proposal was never implemented due to (what seemed in 1984 to be) unfeasibly large storage requirements to store the PDFs. In fact, with cubical geometry, the number of PDFs needed is rather large - for each incident condition, separate PDFs must be generated for at least two exiting direction cosines and two location variables.

The Macro Monte Carlo method was implemented more recently (1991) by Neuenschwander and Born [44], who reduced the storage required by using a more symmetric spherical local geometry. The spherical volume elements were dubbed "kugels". (Kugel means sphere in German.) Several other clever enhancements have made this method extremely fast and a viable option for clinical electron simulation [45].

The improved MMC algorithm features a variety of possible kugel sizes, so that smaller step sizes can be taken as a significant boundary is approached, which allows the user to get accuracy where it is important, yet take large, efficient step sizes through the homogeneous portions of the patient or phantom, as shown in Figure 2. In order for this to work efficiently, the volume is pre-processed and a density assigned to each voxel. Although the pre-processing requires an initial 
computational investment, it is returned just a few minutes into the transport calculation.

The MMC algorithm also uses energy partitioning across a boundary to take into account differences in collision stopping powers on both sides of that boundary. This provides a reasonably accurate and very fast way to deposit energy on either side of the boundary.

For each material, kugel size and electron energy, the distributions of primary electrons emerging from the kugel are stored in 100 equi-probable bins for efficient sampling. Thus the determination of particle parameters after an MMC transport step is reduced to determination of a table index, which is much faster than random interpolation of cumulative PDFs.

Secondary energy for both photons and electrons is released into the local kugel and scored "on the fly". Later it is smeared forward in the incident direction, exponentially attenuated and deposited in a post-process ray-tracing step after the actual simulation. Like the pre-processing step, the post-processing step gives a large gain in efficiency. Although this type of secondary transport is extremely quick, it is also probably the most significant limitation on the accuracy of the MMC method. By not explicitly modeling secondary electrons, the MMC code can flatten out discontinuities in dose across interfaces. The secondary electrons are sensitive to changes in the scattering properties of different materials. Neglecting such changes can be important because they create "hot" and "cold" spots in the dose distribution, particularly in the vicinity of bone-tissue interfaces and tissue-air interfaces. Still, this method is superior to most other clinically used methods. 


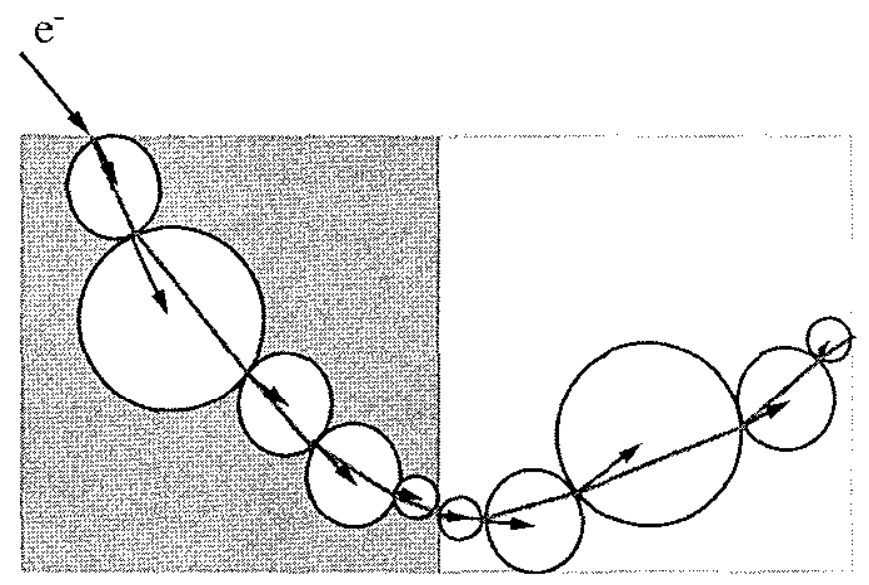

Figure 2: Transport in the Macro Monte Carlo Method with "kugels". The arrows represent the electron trajectory at the source or exit position of the previous step, and the line through the kugels represents the energy deposition path, which extends from the incident point to the sampled exit point.

\subsubsection{Response History Monte Carlo}

\subsubsection{Background}

The Response History Monte Carlo (RHMC) method, although pursued completely independently from the Macro Monte Carlo method, shares its underlying philosophy and a few of its design features. It was developed at Lawrence Livermore Laboratory (LLNL) in 1991 by Ballinger [3], a student from the University of Michigan working with Cullen, Perkins and Rathkopf of LLNL. It began as a marriage of an obscure "response matrix" Monte Carlo algorithm [59] and a Class I condensed history algorithm. 


\section{Local Calculation}

The local geometry in RHMC is a hemisphere, which was chosen because it was believed to be more suitable than a sphere for two reasons. First, the hemisphere easily matches boundary conditions for normally incident electron beams in the global calculation. Secondly, it avoids tracking backscattered electrons in the local calculation. These backscattered electrons add to the computation time without contributing much information to the overall probability distribution being generated; it is more efficient to tally them immediately as they cross the planar boundary. In addition, the hemisphere still allows a modest degree of symmetry for decoupling the energy and angle distributions. ${ }^{7}$

As with the MMC code, the RHMC code recognizes the competing interests at stake in the decision of local geometry size (step size); namely, the larger the size the worse the spatial resolution of the result, but the faster the global calculation can proceed.

The local calculation in RHMC is done using an analog (single scatter) code written explicitly for this use by Ballinger, rather than generating a PDF library from a condensed history code as was done in the Macro Monte Carlo method. The analog code is based entirely on LLNL databases for cross sections, rather than on empirical approximations as is condensed history. Thus the RHMC method has the potential to replicate the accuracy of high-quality experimental and evaluated data.

\footnotetext{
${ }^{7}$ This means that the electrons that reach the surface of the hemisphere have traveled approximately the same distance, so that the electrons leaving the curved surface have then suffered approximately the same number of collisions so energy and trajectory are only loosely related and can be treated independently. The backscattered electrons are considered a completely separate case in this method.
} 


\section{Treatment of Secondary Particles}

Unlike typical Class I transport algorithms, RHMC does permit knock-on electrons to be tracked as regular (primary) histories. The knock-on electrons are recorded in separate PDFs during the local calculation, and these distributions are sampled in the global calculation to determine the "birth state" of the knock-on. There is no capability to model bremsstrahlung in the RHMC algorithm, probably because it was designed for use in low-energy regimes where bremsstrahlung interactions do not account for an appreciable fraction of the total cross section.

\section{Strengths of Response History}

The most significant strength of RHMC is its analog-type accuracy at a fraction of the time required to do an analog calculation. Since the hemispheres are quite small in size, it is able to attain excellent spatial resolution. It is one of the few codes in existence that has been shown to model backscatter realistically, which has great appeal for radiotherapy applications that involve inhomogeneities.

\section{Weaknesses of Response History}

The RHMC code was designed as a proof-of-concept type code, and is not suitable for radiotherapy simulation in its present form for several reasons. First, the energy range of its database is not well-represented at energies above $1 \mathrm{MeV}$, since RHMC was designed to perform well at lower energies. Secondly, the only materials that are immediately available in a form that can be read by the code are aluminum and gold. These elements were chosen as an example of high $\mathrm{Z}$ and relatively low $\mathrm{Z}$ materials (by nuclear transport physics standards) for which an extensive amount of experimental data for benchmarking was readily available. A much more serious limitation is that the RHMC code does not explicitly model bremsstrahlung events, since it handles this energy loss in a Class I - type manner (i.e., the energy is locally 
deposited, which is not a good approximation for photons).

\subsection{Comparison of the Monte Carlo Methods}

Like everything else, finding the best Monte Carlo method is an exercise in weighing tradeoffs. Methods that are fast are often less accurate, and methods that are accurate tend to be slow. There is also an intermediate, the Local-to-Global Monte Carlos, which are relatively fast and accurate, but which require a substantial amount of storage space in compensation.

\subsubsection{Speed}

In general, for Monte Carlo codes, the time required to complete a run on a given platform can be expressed as

$$
T_{\text {run }}=t_{\text {step }} * n_{\text {steps } / \text { history }} * n_{\text {histories }} \text {. }
$$

Assuming the $n_{\text {histories }}$ is constrained by the statistical accuracy required for the answer (although variance reduction methods can decrease this factor), the fastest codes will be those which economize on the time required for each step and/or require less steps for each history.

The Macro Monte Carlo method was built to require fewer steps per particle history, which allows it to excel in speed. The time per step is also minimal, since it consists mainly of sampling PDFs rather than doing calculations. The condensed history methods are intermediate in speed, requiring times that are still generally too long for clinical use, but within the realm of possibility in the next few years, especially if special hardware is implemented and/or variance reduction and other statistical biasing can be used. Response History Monte Carlo run times are on 
the order of that required for condensed history (primarily due to the small step sizes used), however RHMC is more accurate.

\subsubsection{Accuracy}

All of these codes perform approximately equally well in terms of accuracy for a homogeneous phantom. It is more interesting and realistic to benchmark inhomogeneous phantoms. The condensed history methods lose accuracy when the energy range of interest extends below about $100 \mathrm{keV}$ and in regions around an interface differing in atomic number [3] due to underestimation of backscatter. The Macro Monte Carlo method suffers from the same shortcoming as long as it is using probability distribution functions (PDFs) generated by a condensed history code (at present its benchmarks were done using EGS4 data). It should be pointed out that this method has the potential to read PDFs from other, more accurate sources as well, although it still has other shortcomings in this area, like its treatment of secondaries and its large kugel sizes (on the order of several millimeters). The Response History method, on the other hand, was especially designed for highaccuracy results. The fact that it is based on a single scatter code and that its "local geometry" (hemispheres) is relatively small (on the order of microns) gives it an advantage over the other methods, especially at low incident energies and when backscatter from interfaces is important. The most accurate simulation method available today is the analog or single scatter method [4].

\subsubsection{Storage}

There are two factors that affect the storage and/or size of a code: versatility and speed. The dramatic speed-up of the RHMC and MMC methods over single scatter and condensed history codes, respectively, are obtained at the expense of storing the pre-calculated results of another slower code. However, both of these 
Table 1: A comparision of various features of electron transport in Monte Carlo codes introduced in Chapter 1.

\begin{tabular}{|c|c|c|c|c|}
\hline Algorithm & $\begin{array}{c}\text { Condensed History } \\
\text { Class II }\end{array}$ & $\begin{array}{c}\text { Condensed History } \\
\text { Class I }\end{array}$ & $\begin{array}{l}\text { Local to } \\
\text { Global }\end{array}$ & $\begin{array}{l}\text { Local to } \\
\text { Global }\end{array}$ \\
\hline Code & EGS4 & ETRAN/MCNP4A & $\mathrm{MMC}$ & RHMC \\
\hline Angular & Moliere & Goudsmit-Saunderson & from & from \\
\hline Scatter & $>20$ events & No small $\theta$ approx & EGS4 & Analog \\
\hline Model & small $\theta$ approx. & Legendre poly. & & code \\
\hline Energy & restricted stopping & Landau Distribution & from & from Analog \\
\hline Loss & power & assumes $E_{b i n d}<<E$ & EGS4 & code \\
\hline \multirow{3}{*}{ Location } & straight- & straight- & from & from \\
\hline & line & line & & Analog \\
\hline & approximation & approximation & EGS4 & code \\
\hline Secondary & generated by & none, but & smeared forward & same as primary \\
\hline \multirow[t]{2}{*}{ Electrons } & Bhabha-Moller & $\mathrm{E}$ loss included in & at end of & if escape \\
\hline & cross-sections & Landau distribution & simulation & local geometry \\
\hline Step Size & variable steps: & steps of & spheres: & hemispheres \\
\hline and Shape & $1 \%-20 \% \mathrm{E}$ loss & predetermined length & $\mathrm{r}=.05-.3 \mathrm{~cm}$ & $10^{-7}-10^{-3} \mathrm{~cm}$ \\
\hline Energy & $100,000 \mathrm{MeV}$ - & $100 \mathrm{MeV}$ - & $20 \mathrm{MeV}$ & $200 \mathrm{keV}-$ \\
\hline Range & $10 \mathrm{keV}$ & $100 \mathrm{keV}$ & $200 \mathrm{keV}$ & binding energy \\
\hline
\end{tabular}

codes were built for specific applications, while most other condensed history codes are large in order to be useful in a variety of situations. Furthermore, the cost of memory is decreasing at such a fast rate (about a factor of two every five years) that this concern is probably not as binding as the concerns of speed and accuracy.

\subsubsection{Summary}

The characteristics of the various algorithms are briefly summarized in Table 1. 


\subsection{Recent Developments}

There have been at least two recent efforts to make Monte Carlo calculations routinely available for radiation treatment planning.

\subsubsection{The OMEGA Project}

The Ottawa - Madison Electron - Gamma Algorithm (OMEGA) project is a multiyear study undertaken jointly by the University of Wisconsin - Madison and National Research Council of Canada (NRCC). The purpose of the project is to routinely calculate the dose from electron beams in clinical-type situations using Monte Carlo. Presently, this is accomplished primarily through the EGS4 code.

The OMEGA group has broken down the problem of radiotherapy dosimetry into two specific areas: (1) characterizing the beam as it exits the accelerator and passes an arbitrary scoring plane, which is different for each treatment machine but the same from patient to patient; and (2) transporting the radiation from this scoring plane through beam shaping devices, which may change with each treatment, and patient anatomy. This division is illustrated in Figure 3.

In order to transport the electron beam through the treatment head of the accelerator, a new EGS4-based code system, BEAM, was written. BEAM provides information about both the incident energy and the angular distribution of electrons at the scoring plane. Each head configuration can be simulated once, then the histories can be started at the scoring plane. The second part of the project has resulted in a Monte Carlo code, DOSXYZ, integrated with a $3 \mathrm{D}$ radiotherapy treatment planning system which was developed at the University of Wisconsin. Much of the work on the MMC code, discussed in Section 1.6.1, was carried out as part of the OMEGA project. These tools are presently being used for electron-photon transport in patient volumes and test phantoms. 

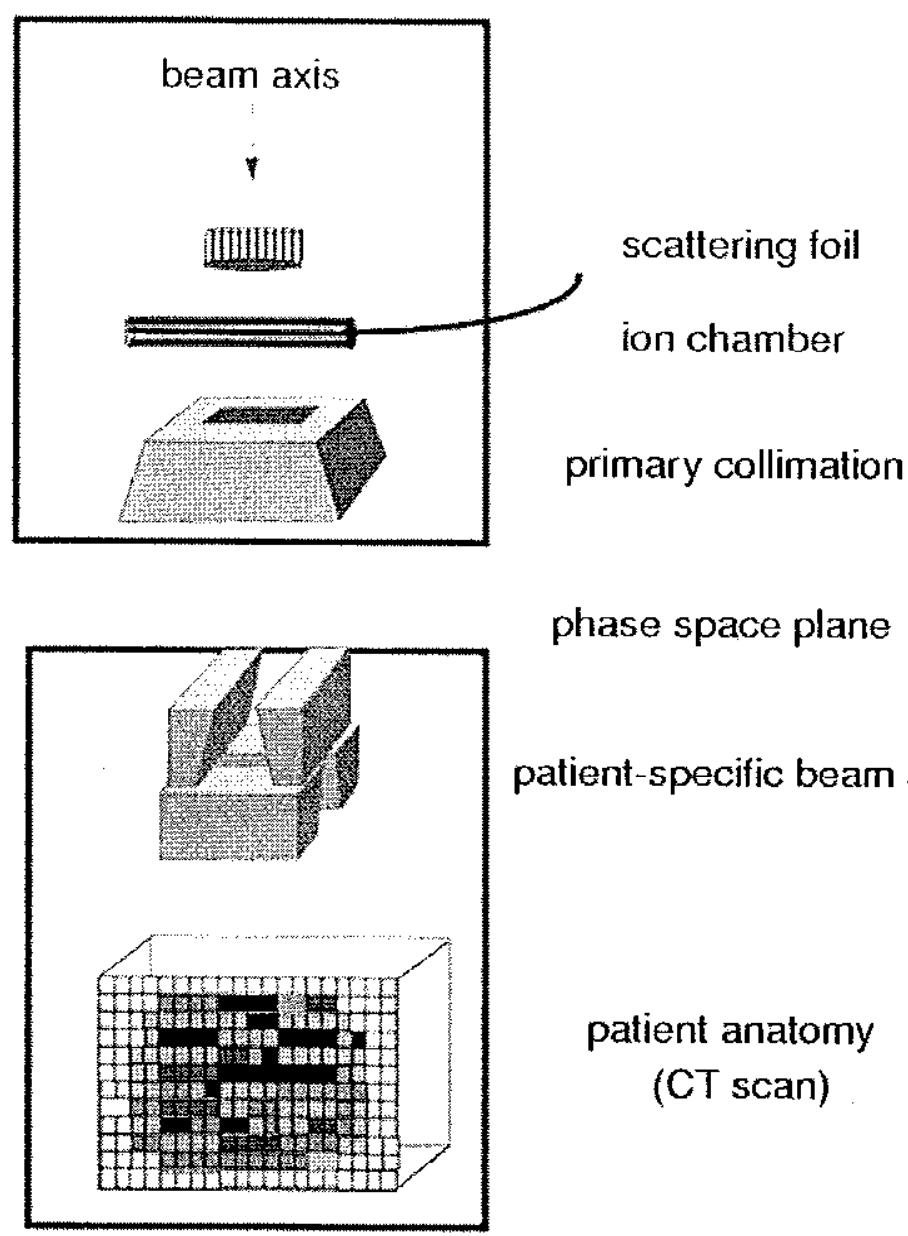

phase space plane

patient-specific beam shaping

patient anatomy

(CT scan)

Figure 3: Two separate portions of the problem of radiotherapy simulation. 


\subsubsection{The Peregrine Project}

Peregrine is a 3-dimensional Monte Carlo code developed at LLNL specifically to address the problem of radiotherapy simulation. It was designed to track particles through a regular Cartesian mesh based on the CT scan, at the same resolution. It is intended to be computationally fast enough to be acceptable for routine dose calculations.

Peregrine breaks the problem of radiotherapy simulation down in a manner similar to that of OMEGA, and has source models which are derived in a separate computation from OMEGA's BEAM code and MCNP4B $[51,16]$. The source models approximate the spectral output of the accelerator at an arbitrary transverse plane downstream of the electron target, flattening filter, primary collimator, and monitor chamber.

The simulation proceeds by sampling the energy and trajectory of an emitted particle, doing simplified transport through the collimators and any other apertures, blocks, or wedges, until the particle reaches the the patient, where Monte Carlo calculation of dose deposition is done.

Peregrine is designed to run on its own hardware in conjunction with an existing client treatment planning system.

\subsection{Scope of this Work}

The initial impetus of this work was to see if Peregrine, like OMEGA, could benefit from a Local-to-Global approach to electron transport.

The process will be unfolded in the chapters to come. Basically, the MRMC method combines the strengths of the previous work in this area. The local stage is based largely on Ballinger's work. The geometry was changed from hemispheres to spheres, a wider range of materials was included, a newer version of the EEDL 
database was used, and the benchmarking emphasis was on energy deposition in slabs as opposed to low-energy phenomena like backscatter.

The global stage was based largely on Neuenschwander's work, with some differences. The MRMC stepping routines were coupled to a version of Peregrine for gamma transport. The electrons are incident in the center of the kugel, rather than the edge, for better symmetry. Secondary electrons that escaped the kugel are transported in the same manner as primaries. The energy deposition algorithm was designed to spread energy out across many zones, to somewhat compensate for the increased CPU time required by explicit transport of secondaries.

Chapter 2 is a description of the first step in designing a new Local-to-Global electron transport package with single scatter physics as its foundation. The code that was written to fill this need, CREEP (Code for Reconstruction of Exact Electron Progression), is introduced. The physics it simulates, the assumptions it contains, the logic flow, and the results obtained with it are discussed in detail.

Chapter 3 will discuss the MRMC library - the collection of kugel data sets generated by CREEP. It details the quantities selected for tallying, the binning of the kugel surface and the binning of the individual probability distributions. The layout of the library is shown, as well as several examples of methods to run multiple kugel sizes efficiently. Memory requirements are also discussed.

Chapter 4 discusses a practical, efficient application of CREEP through a global stepping code. The logic flow, the quantities sampled from the kugel data library, and various algorithms for energy deposition and boundary crossing are discussed in detail.

Chapter 5 shows a series of test problems that were designed to evaluate specific features of this method. The depth dose results for several simple phantoms obtained by MRMC and condensed history Monte Carlo are shown and analyzed.

Chapter 6 contains the summary and conclusions of this research, as well as some suggestions for future work. 


\section{Chapter 2}

\section{Single Scatter Electron Monte Carlo}

\subsection{Introduction}

This chapter describes the single scatter Monte Carlo (SSMC) code, CREEP, that serves as the local phase of MRMC transport. Although its primary purpose is to compile the probability distribution functions (PDFs) necessary for the global phase of MRMC transport, the code has also proven to be interesting in its own right. The following sections describe the physics algorithms and databases used by CREEP, as well as a number of results that were obtained with the code.

\subsubsection{Why Single Scatter?}

Single scatter physics is gaining attention for electron transport, despite the fact that it is inherently very time consuming. One reason is that since single scatter calculations conform (more) closely to the physical processes the electron undergoes, they can serve as a means to explore the validity of assumptions used in other transport techniques. In particular, single scattering can help make efficient electron transport methods, like condensed history and local-to-global methods, more accurate.

SSMC allows large angle scatter and backscatter measurements to be calculated with greater accuracy in a reliable manner. Large angle scatter and backscatter, 
being relatively rare, result in much of the seemingly eccentric energy deposition behavior of electron beams (and photon beams for that matter, since photons deposit their energy to the medium through secondary electrons), including lateral blooming with distance and nonuniformities ("hot" or "cold" spots) found near changes in the medium type or density.

\subsubsection{The EEDL Database}

The Evaluated Electron Data Library (EEDL) was established at LLNL by 1990 to complement the ENDL (Evaluated Nuclear Data Library) and EPDL (Evaluated Photon Data Library). Complete documents detailing its contents, with derivations, are available $[17,48,49]$. Cross sections for each atomic subshell, for each interaction, are tabulated on an energy grid with a variable placement of points between $10 \mathrm{eV}$ and $100 \mathrm{GeV}$, for atomic numbers 1 to 100 .

The elastic scattering cross sections are based on those of Mott for energies greater than $256 \mathrm{keV}$ and of Riley below $256 \mathrm{keV}$. These data were then extrapolated to cover the entire energy range. Spectra, in the form of probability distribution functions (PDFs), of angular deflections for a variety of incident energies are also tabulated.

The impact ionization cross sections are based on the Moller formalism with other corrections to accurately model small energy loss collisions. Energy loss spectra are available at a number of incident energies for individual ionization and bremsstrahlung events, as well as the spectral average energy loss.

The bremsstrahlung cross sections were determined by Seltzer and Berger [54] by interpolating between the relativistic data from the code of Tseng and Pratt [58] available up to $2 \mathrm{MeV}$, and the results of Bethe-Heitler, expected to be valid above $50 \mathrm{MeV}$.

The excitation database contains cross sections and the average energy loss to 
excitation as a function of incident energy. There are no spectral data for excitation energy loss in EEDL at this time. A summary of the database features that were used at some point in CREEP can be seen in Table 2 and Table 3.

It should by noted that an independent, simpler electron single scatter code, also based on the EEDL data, was written by Ballinger [4] in 1991 at LLNL and provided a strong foundation for this work.

\subsection{Physics Algorithms}

The CREEP code is written in FORTRAN and $\mathrm{C}$, in a very simple style with the intent of being extremely portable. Since this code is intended primarily as a means to explore basic physical properties of the medium, the present incarnation assumes only simple geometries: either spherical (user specifies radius) or slab (user specifies $\mathrm{x}, \mathrm{y}, \mathrm{z}$ ), consisting of one type of material. Several slabs may be pieced together to simulate a layered geometry, since the output of one slab may be used as spectral input into a neighboring slab, and the backscattered energy spectrum from the each interface can be transported backwards into the prior medium.

\subsubsection{Simulating Individual Electron Interactions in Media}

The algorithm for a single scatter charged particle code is basically the same as the algorithm that has historically been used in photon and neutron Monte Carlo codes. Briefly, one finds the distance to interaction by finding the total cross section at the present energy and uses the relation $s=-\lambda \ln (\chi)$, where $\chi$ is a random number on the interval $(0,1)$. One then determines which interaction took place, by forming and sampling from a cumulative probability based on the cross sections for each of the four possible interactions (ionization, excitation, elastic scatter, bremsstrahlung). The energy, position and trajectory of the particle is 
Table 2: Some relevant contents of the EEDL database for an element. Elements with atomic numbers 1 - 99 are available in the database. All energies are in $\mathrm{MeV}$; cross sections are in barns. $P$ is the differential probability of the particle out for each of the appropriate distributions. The integer labels represent flags EEDL uses to identify the interaction, data type, and outgoing particle.

\begin{tabular}{|c|c|c|c|}
\hline Interaction & Type of Data & Particle Out & EEDL Columns \\
\hline $\begin{array}{c}8 \\
\text { elastic scatter }\end{array}$ & $\begin{array}{l}0 \text { - Integrated elastic } \\
\text { cross section }\end{array}$ & $\begin{array}{c}0 \\
\text { (none) }\end{array}$ & $E_{i n}, \sigma$ \\
\hline $\begin{array}{c}8 \\
\text { elastic scatter }\end{array}$ & $\begin{array}{l}10 \text { - average energy } \\
\text { given to residual atom }\end{array}$ & $\begin{array}{c}11 \\
\text { residual atom }\end{array}$ & $E_{\text {in }},<E_{\text {loss }}>$ \\
\hline $\begin{array}{c}8 \\
\text { elastic scatter }\end{array}$ & $\begin{array}{c}10 \text { - average energy } \\
\text { after event }\end{array}$ & $\begin{array}{c}9 \\
\text { "primary" e- }\end{array}$ & $E_{\text {in }},<E_{\text {out }}>$ \\
\hline $\begin{array}{c}8 \\
\text { elastic scatter }\end{array}$ & $\begin{array}{c}21 \text { - scatter angle } \\
\text { distribution }\end{array}$ & $\begin{array}{c}9 \\
\text { "primary" e- }\end{array}$ & $E_{\text {in }},<1-\cos \theta>, \mathrm{P}$ \\
\hline $\begin{array}{c}81 \\
\text { ionization }\end{array}$ & $\begin{array}{l}0 \text { - Integrated ionization } \\
\text { subshell cross section }\end{array}$ & $\begin{array}{c}0 \\
\text { (none) }\end{array}$ & $E_{\text {in }}, \sigma$ \\
\hline $\begin{array}{c}81 \\
\text { ionization }\end{array}$ & $\begin{array}{c}10 \text { - average energy } \\
\text { after event }\end{array}$ & $\begin{array}{c}9 \\
\text { "primary" e- }\end{array}$ & $E_{\text {in }},<E_{\text {out }}>$ \\
\hline $\begin{array}{c}81 \\
\text { ionization }\end{array}$ & $\begin{array}{c}10 \text { - average energy } \\
\text { after event }\end{array}$ & $\begin{array}{c}19 \\
\text { "knock-on" e- }\end{array}$ & $E_{i n},<E_{k o n}>$ \\
\hline $\begin{array}{c}81 \\
\text { ionization }\end{array}$ & $\begin{array}{l}21 \text { - spectrum of } \\
\text { secondary energy }\end{array}$ & $\begin{array}{c}19 \\
\text { "knock-on" e- }\end{array}$ & $E_{\text {in }},<E_{k o n}>, \mathrm{P}$ \\
\hline
\end{tabular}


Table 3: Some relevant contents of the EEDL database for an element. Elements with atomic numbers 1 - 99 are available in the database. All energies are in $\mathrm{MeV}$; cross sections are in Barns. $P$ is the differential probability of the particle out for each of the appropriate distributions. The integer labels represent flags EEDL uses to identify the interaction, data type, and outgoing particle.

\begin{tabular}{|c|c|c|c|}
\hline Interaction & Type of Data & Particle Out & EEDL Columns \\
\hline $\begin{array}{c}82 \\
\text { bremsstrahlung }\end{array}$ & $\begin{array}{l}0 \text { - Integrated brem } \\
\text { cross section }\end{array}$ & $\begin{array}{c}0 \\
\text { (none) }\end{array}$ & $E_{i n}, \sigma$ \\
\hline $\begin{array}{c}82 \\
\text { bremsstrahlung }\end{array}$ & $\begin{array}{c}10 \text { - average energy } \\
\text { after event }\end{array}$ & $\begin{array}{c}9 \\
\text { "primary" e- }\end{array}$ & $E_{\text {in }},<E_{\text {out }}>$ \\
\hline $\begin{array}{c}82 \\
\text { bremsstrahlung }\end{array}$ & $\begin{array}{c}10 \text { - average energy } \\
\text { after event }\end{array}$ & $\begin{array}{c}7 \\
\text { brem photon }\end{array}$ & $E_{\text {in }},<E_{p h o}>$ \\
\hline $\begin{array}{c}82 \\
\text { bremsstrahlung }\end{array}$ & $\begin{array}{l}21 \text { - spectrum of } \\
\text { secondary energy }\end{array}$ & $\begin{array}{c}7 \\
\text { brem photon }\end{array}$ & $E_{i n},\left\langle E_{p h o}\right\rangle, \mathrm{P}$ \\
\hline $\begin{array}{c}83 \\
\text { excitation }\end{array}$ & $\begin{array}{c}0 \text { - Integrated excitation } \\
\text { cross section }\end{array}$ & $\begin{array}{c}0 \\
\text { (none) }\end{array}$ & $E_{\text {in }}, \sigma$ \\
\hline $\begin{array}{c}83 \\
\text { excitation }\end{array}$ & $\begin{array}{l}10 \text { - average energy } \\
\text { given to residual atom }\end{array}$ & $\begin{array}{l}11 \\
\text { residual atom }\end{array}$ & $E_{\text {in }},<E_{\text {loss }}>$ \\
\hline
\end{tabular}


updated to reflect the chosen interaction in a manner described for each below. Then the same process is begun again, provided the electron has not escaped the medium or fallen below the energy cutoff.

CREEP is actually a family of four codes, having a similar ancestor code but they have evolved separately to fill specific niches. SlabcreepI, was written for the purpose of benchmarking with slab and foil experiments. SlabcreepII does the same but for media that are not comprised of a single element; it handles compounds and mixtures (see Section 2.2.2) and was primarily designed as a means to compare the CREEP method with other codes and experiments for generating depth-dose curves in water, which is the most important medium for radiotherapy applications. The ultimate application for CREEP was to generate a library for the Macro Response Method (MRMC), for which probability distribution functions arising from transport through a sphere are required. Thus KugcreepI and KugcreepII were born; the former for single-element materials and the latter for compounds and mixtures in spherical geometry. It would have been possible to merge the codes, however, the decision was made to keep them separate in the interest of efficiency.

The flow chart illustrating the general logic of the codes is shown in Figure 4. For both the slab-geometry code and the spherical-geometry kugel code, there are two types of input files. The first is a very simple user-generated file explaining the Monte Carlo tracking parameters, the medium, and the output information desired. The second type of information files required is the EEDL datafile for each element in the medium.

The CREEP code deviates from the ideal single scatter algorithm in that (for most applications) it does not simulate every excitation event individually. Instead, it subtracts off the expected excitation loss after each of the other events, as described in the excitation section below. This choice was made because it was demonstrated that there was little to gain by direct simulation of excitations, since 


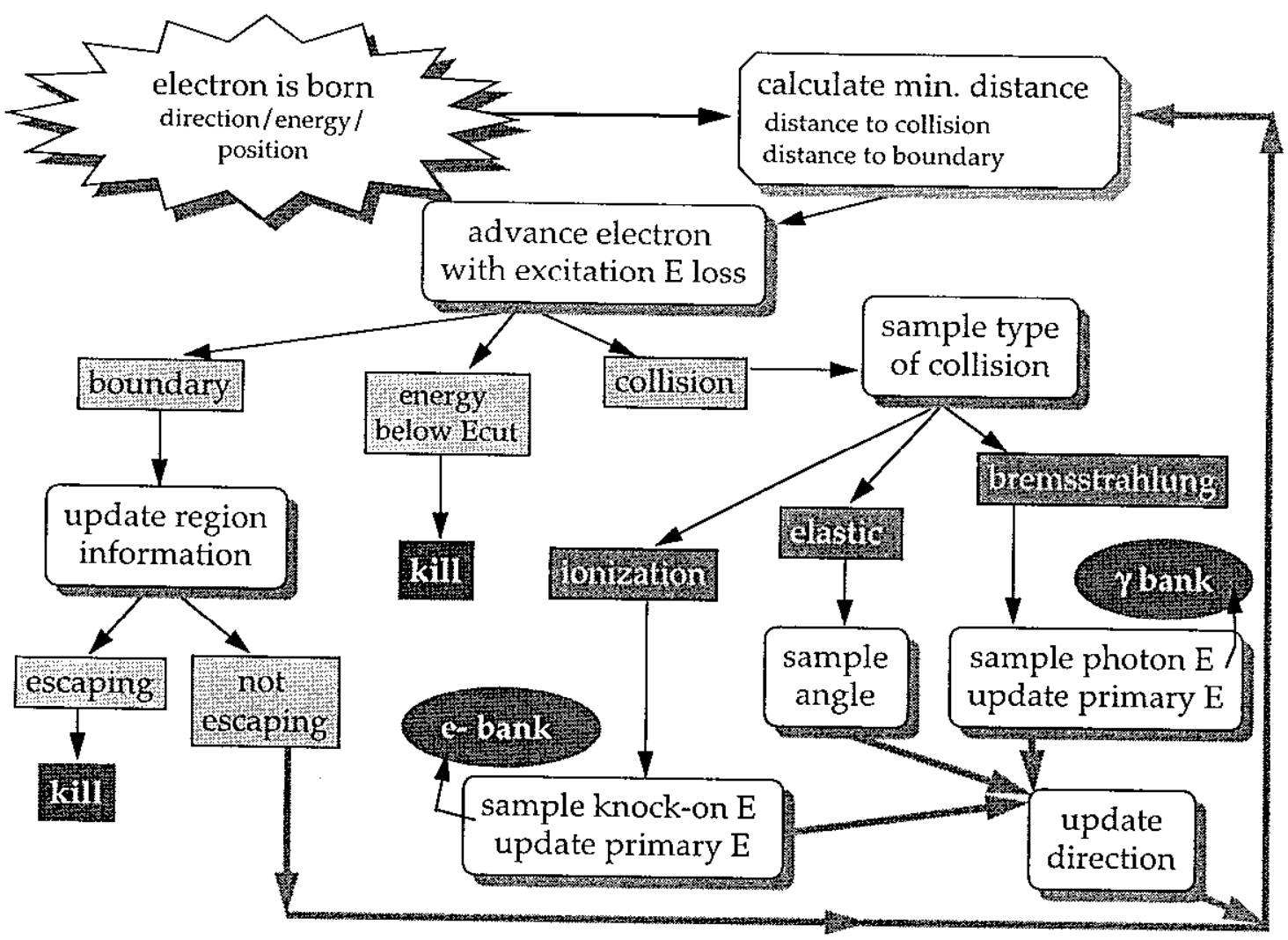

Figure 4: The logic flow of the CREEP analog electron scatter Monte Carlo code. When an electron reaches a "kill" box, its residual energy is deposited locally and another electron is initiated from the bank. 
they do not have a large effect on the electron trajectory, and the amount of the overall energy loss to this mechanism - although it can be large - is predictable. Using the pathlength-based expected loss also makes the results statistically smoother for the same number of histories.

\section{Ionization}

Ionization interactions are a dominant energy loss mechanism for electrons slowing down from the radiotherapy energy range. They occur when a charged particle imparts enough of its kinetic energy to a orbital electron to set that electron free. This process is illustrated in Figure 5. Once the electron is free it is called a "knockon" electron or "delta-ray". For incident electrons, the interaction is often pictured as a "black box" in the vicinity of an atomic electron with two electrons exiting. Because electrons are indistinguishable from each other, it is simply assumed that the electron with the higher exit energy was the primary electron, making the remaining electron the "knock-on". With this definition, the maximum energy a knock-on electron can have is given by

$$
T_{k \text { nock, } \max }^{\prime}=\frac{T_{0}-E_{\text {bind }}}{2}
$$

where $E_{b i n d}$ is the binding energy of the shell from which the knock-on was freed.

To simulate an ionization interaction, the knock-on electron energy is sampled from a spectrum. The EEDL database has a number of spectra tallied for various incident energies; statistical interpolation is used to choose between them. Once the energy of the knock-on has been selected, 2-body kinematics (neglecting binding energy) are used to update the primary electron's trajectory. If $K_{0}=T_{0} / m_{0} c^{2}$ and the ratio $\chi$ is defined by $\chi=T_{\text {knockon }} / T_{0}$, then the outgoing angles are given by 


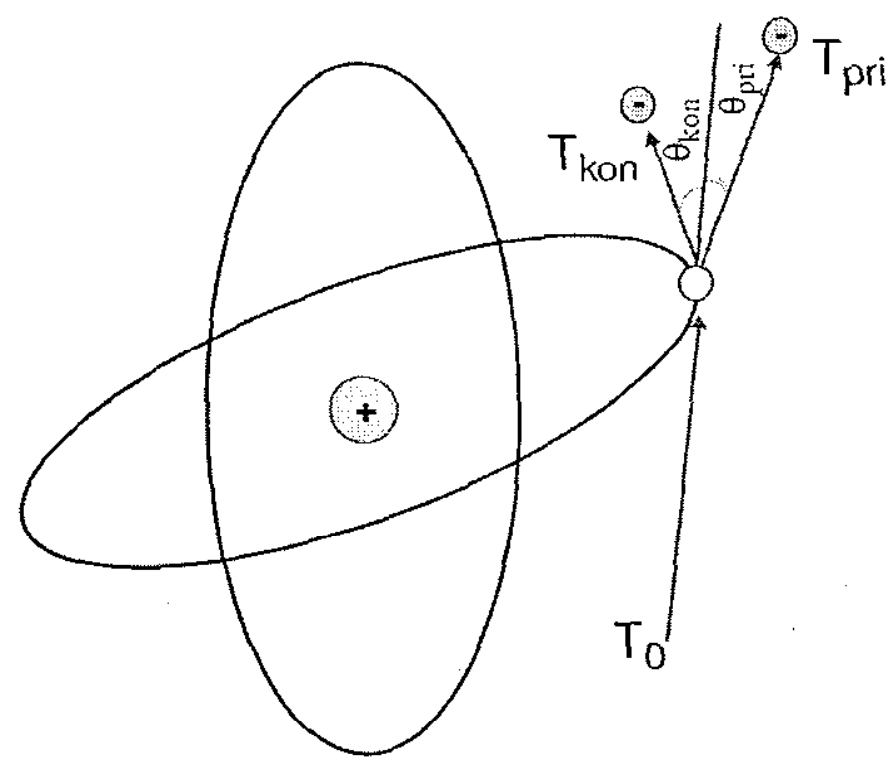

Figure 5: An ionization interaction resulting in the liberation of an atomic electron. The electron is incident with energy $T_{0}$, and exits with $T_{\text {pri }}$ after setting a knock-on of energy $T_{k o n}$. 


$$
\cos \theta_{p r i}=\sqrt{\frac{\chi\left(K_{0}+2\right)}{\chi K_{0}+2}}
$$

and for the knock-on electron

$$
\cos \theta_{k n o c k}=\sqrt{\frac{(1-\chi)\left(K_{0}+2\right)}{(1-\chi) K_{0}+2}}
$$

Note that because the binding energy is neglected, these angles are less valid at energies near it. Since such electrons don't travel far, this assumption does not have a large impact on the overall transport results.

CREEP handles secondary electrons by putting the primary on a memory stack and tracking the knock-on immediately, until they fall below the energy cutoff or escape the volume, at which point the primary history is continued. A special energy cutoff parameter is used for knock-ons, so the user can readily imitate Class II condensed history codes, which only simulate ionization events if the knock-on is above a particular energy.

Presently it is assumed that all binding energy is locally deposited. For arbitrary media, this assumption is weak since significant fractions of the binding energy may be re-emitted as Auger electrons or fluorescent x-rays. Many of the $\mathrm{x}$-rays are emitted at energies that fall just below the photoelectric edges, where the cross-sections are small and they can therefore carry their energy relatively far from the interaction site. However, the atomic de-excitation of low atomic number materials is dominated by Auger electron production, which do not travel far. Since tissue is mainly of low atomic number, the local deposition approximation is justified for this application. Another LLNL database, EADL (Evaluated Atomic 
Data Library) contains all the necessary information to model these relaxations physically, but is not incorporated into the code at this time.

\section{Elastic Scattering}

In elastic scattering interactions, an incident electron traveling in the vicinity of a nucleus scatters off the nucleus at some angle without a significant loss in energy, due to the large mass difference between the two. However, each scatter does cause a (generally) modest angular deflection. This is illustrated in Figure 6. Due to its large cross-section, the net effect of elastic scatter on a particle's trajectory can be very significant, as is demonstrated by Figure 7 .

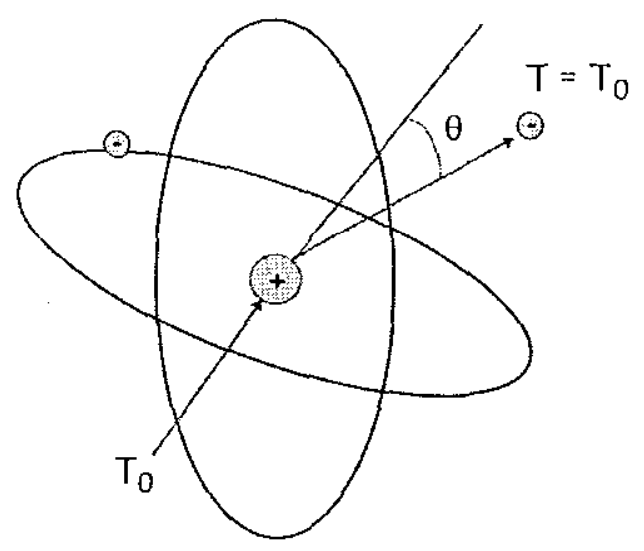

Figure 6: An illustration of an elastic scatter interaction. The electron is deflected through an angle $\theta$ with no energy loss.

The cross section for elastic scattering as a function of solid angle is given by the McKinley-Feshback form of Mott scattering in the expression

$$
\begin{aligned}
\frac{d \sigma_{\text {elas }}}{d \Omega}= & \frac{Z^{2}}{4}\left(\frac{e^{2}}{m_{0} c^{2}}\right)^{2} \frac{1-\beta^{2}}{\beta^{4}} \frac{1}{\sin ^{4}\left(\frac{\theta}{2}\right)} \\
& \times\left[1-\beta^{2} \sin ^{2}\left(\frac{\theta}{2}\right)+\pi \beta \frac{Z}{137}\left(1-\sin \left(\frac{\theta}{2}\right)\right) \sin \left(\frac{\theta}{2}\right)\right]
\end{aligned}
$$




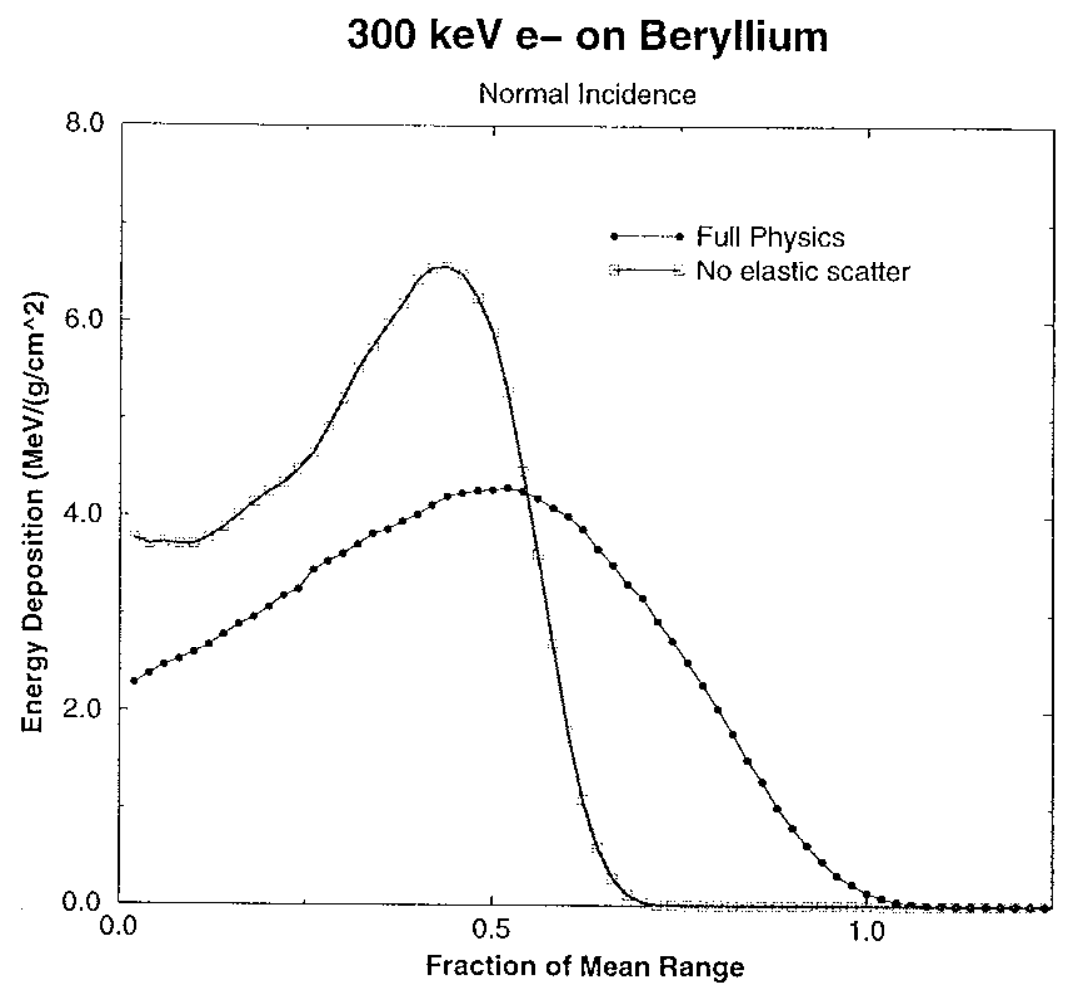

Figure 7: An example of the effect of elastic scatter angles on energy deposition versus depth in beryllium. The "no elastic scatter" curve was made by reassigning the scatter angle to 0 after each elastic event. Its shape approximates the "Bragg peak" formed by heavier charged particles, but with a remaining width that is a function of (1) energy straggling, discussed in Section 1.5.3 and (2) scatter angles due to ionization interactions, given by equations 2.2 and 2.4. The mean range for $300 \mathrm{keV}$ electrons in beryllium is $0.1037 \mathrm{~g} / \mathrm{cm}^{2}$. 
where $Z$ is the nuclear charge, $e$ is the electron charge, and the term outside of the square brackets is the Rutherford scattering term. Note that the cross section depends inversely on the square of the rest mass of the particle, so heavy charged particles, like protons or ions, scatter much less than electrons and positrons.

To simulate this event, one samples a scattered angle from EEDL (where it is tabulated as $1-\cos \theta)$ and updates the trajectory. Although most elastic scattering results in only a small angle, it is this mechanism that is largely responsible for the phenomena of backscatter and large angle scatter.

\section{Bremsstrahlung}

Bremsstrahlung interactions occur when the electron passes near the nucleus and accelerates due to the interaction of their Coulomb fields, causing a photon to be emitted. A schematic of this process is shown in Figure 8. Although low energy photons are more likely, an electron can lose up to all of its energy to the photon. As an energetic charged particle of mass $m_{0}$ and charge $z e$ passes in the vicinity of a nucleus of mass $M_{n}$ and charge $Z e$, there will be an electrostatic force between the two particles due to the interaction of the Coulomb fields, given by

$$
F_{E}=\frac{\kappa z Z e^{2}}{r^{2}}
$$

The incident charged particle will experience an acceleration due to this force of magnitude

$$
a=\frac{\kappa z Z e^{2}}{r^{2} m_{0}}
$$


where $r$ is the separation between the particles and $\kappa$ is a fundamental electromagnetic constant $=\left(4 \pi \epsilon_{0}\right)^{-1}$. Assuming the mass of the charged particle is small compared to the nucleus, the nucleus does not move significantly as a result of the force in equation 2.7. However, the force will cause the charged particle to be deflected from its path and momentarily orbit around the nucleus. An accelerated charge radiates energy at a rate proportional to the square of its acceleration

$$
\left.\frac{d T}{d t}\right|_{b r e m} \propto\left(\frac{\kappa z Z e^{2}}{r^{2} m_{0}}\right)^{2}
$$

Equation 2.9 illustrates several important concepts governing bremsstrahlung emission. First, it is apparent that it is much more common for light particles such as electrons to emit photons than it is for heavier particles like protons, due to the $1 / m_{0}^{2}$ dependency. Secondly, it can be seen that bremsstrahlung is much more important in high atomic number materials (due to the $Z^{2}$ ) than in low atomic number materials. In principle, it is possible to have bremsstrahlung created in the field of an atomic electron, but the probability is much lower, since the charge (and therefore the acceleration) is less.

A more exact equation for the differential cross section was derived by Schiff [52]. The basic dependencies shown in equation 2.9 can be seen in his result, which is expressed in terms of a reduced angle, $x=T_{0} \theta_{0} / m_{0} c^{2}$, where $\theta_{0}$ is the angle between the photon and the incident electron trajectories:

$$
\begin{aligned}
\sigma_{b}(k, x)= & \frac{4 Z^{2}}{137}\left(\frac{e^{2}}{m_{0} c^{2}}\right)^{2} \frac{d k}{k} x d x\left\{\frac{16 x^{2} T}{\left(x^{2}+1\right)^{4} T_{0}}\right. \\
& \left.-\frac{\left(T_{0}+T\right)^{2}}{\left(x^{2}+1\right)^{2} T_{0}^{2}}+\left[\frac{T_{0}^{2}+T^{2}}{\left(x^{2}+1\right)^{2} T_{0}^{2}}-\frac{4 x^{2} T}{\left(x^{2}+1\right)^{4} T_{0}}\right] \ln M(x)\right\},
\end{aligned}
$$




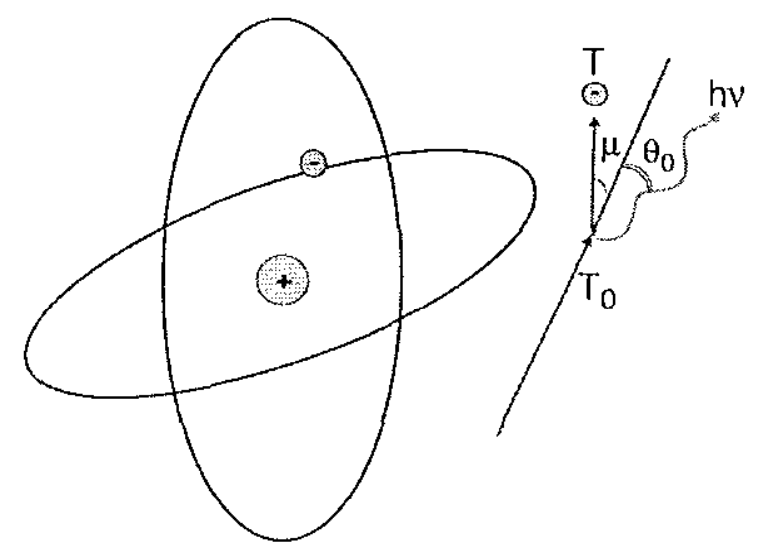

Figure 8: A electron giving rise to a bremsstrahlung photon in the field of a nucleus. The electron is incident with energy $T_{0}$ and scatters through an angle $\mu$ with outgoing energy $T$ creating a photon of energy $h \nu$.

where

$$
\frac{1}{M(x)}=\left(\frac{m_{0} c^{2} k}{2 T_{0} T}\right)^{2}+\left(\frac{Z^{1 / 3}}{111\left(x^{2}+1\right)}\right)^{2}
$$

and $k=h \nu=T_{0}-T$, the energy of the bremsstrahlung photon.

The exact rate of energy loss by bremsstrahlung depends on the quantum mechanical nature of this interaction, which is beyond the scope of this work (see, for example, Tsai [57]). However, for energies less than $100 \mathrm{MeV}$, the energy loss may be estimated by the equation

$$
\frac{1}{\rho}\left(\frac{d T}{d x}\right)_{\mathrm{rad}}=4 r_{0}^{2} \frac{N_{e} Z T_{0}}{137}\left[\ln \frac{2\left(T_{0}+m_{0} c^{2}\right)}{m_{0} c^{2}}-\frac{1}{3}\right]
$$

where $N_{e}=\left(N_{A} Z\right) / A$, which is the number of electrons per gram, $T_{0}$ is the kinetic energy of the incident electron, and $r_{0}$ is the classical electron radius $\left(r_{0}=2.81794 \times 10^{-15} \mathrm{~m}\right)$. The important physics revealed by this equation is 
that energy loss increases directly with atomic number of the material, and the loss increases to a somewhat greater extent with the energy of the electron.

In bremsstrahlung interactions, the initial momentum of the incident particle becomes shared between the scattered charged particle, the atomic nucleus and the emitted photon. Therefore, the photon can have any energy up to $h \nu_{\max }=T_{0}$. In this manner charged particles, especially electrons, have a small probability of losing almost all of their energy in a single interaction, however, this only occurs at extreme relativistic energies. At such energies, both the photon and the scattered charged particle advance preferentially in the forward direction. For moderate energy charged particles, however, the photon carries only a very small momentum and can be emitted in any direction.

In CREEP, the photon energy is sampled from a spectrum, and an empirical relation can be used to determine the cosine of the scatter angle, $\cos \mu$, the electron travels through as a result of the interaction:

$$
\cos \mu=1-\left(\frac{m_{0} c^{2}}{T_{0}-h \nu}-\frac{m_{0} c^{2}}{T_{0}}\right)
$$

where $T_{0}$ is the kinetic energy of the incident electron at interaction site, i.e., the energy lost to excitations from the previous step to the present location has been subtracted.

The "birth" angle of the bremsstrahlung photon is more difficult; it is most correctly obtained by sampling the Schiff formula [52], but CREEP uses the approximation

$$
\theta_{0}=\frac{m_{0} c^{2}}{m_{0} c^{2}+T_{0}}
$$

where the denominator represents the total energy of the electron at the site before the event. 
CREEP itself does not track the bremsstrahlung photons that are created; they are tallied on the spot so that their phase space can be banked and passed off to another Monte Carlo code with photon tracking capabilities. Note that any additional electrons the bremsstrahlung photons would have generated are lost, so CREEP cannot assume any energy deposits arising from photons. If, however, CREEP is coupled with a photon MC code in a way that allows that code to pass back further secondary electrons these can be restored. It should be noted that the bremsstrahlung photon is much more penetrating than the charged particle that caused it, and therefore carries its energy far from the original charged particle track. Monte Carlo codes that neglect bremsstrahlung interactions thus fail to model this energy deposition pattern accurately.

\section{Excitation}

The primary charged particle can excite an atom even thought it does not impart enough energy to the atomic electron to free it. Instead, the energy transferred to the atom causes the orbital electron to be promoted to a higher electronic state. This is illustrated in Figure 9. The promoted orbital electron relaxes either by producing characteristic radiation of energy $h \nu$; or by producing Auger electrons of energy $\mathrm{h} \nu-E_{\text {binding; }}$; or transferring the energy to molecular vibrational modes at higher energy. Since the energies involved are typically very low compared to the energy range of interest, the individual events are often not modeled and the energy that is given to them is instead considered to be locally deposited. In fact, these events may be lumped together and assumed to cause a uniform energy loss per unit distance. This is an "excitation-only" stopping power.

In CREEP, the energy loss due to excitations can be accounted for by finding the total excitation cross section at current energy, and using the mean energy lost to excitation events at that energy to construct an excitation-only stopping power 


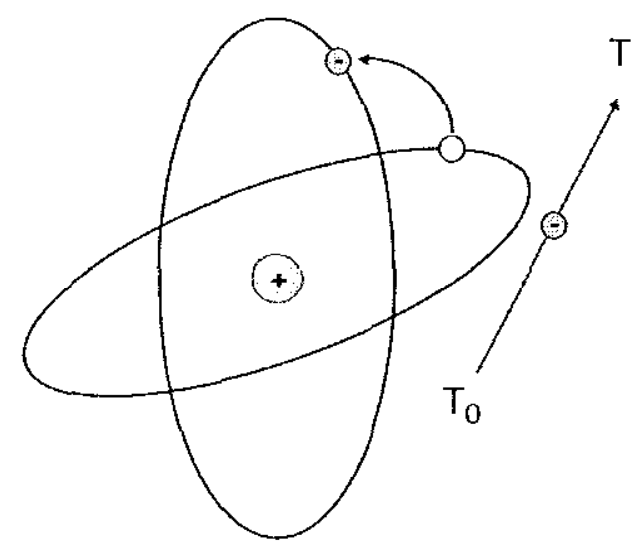

Figure 9: A incident electron causing an atomic electron to become promoted into a higher electronic state. The incident electron has given up a small amount of its energy, $T_{0}-T$, equal to the difference in energy levels. Some of this energy may be re-emitted as Auger electrons or fluorescent photons.

through the relation

$$
\left(\frac{d E}{d x}\right)_{e x}=\frac{N_{A}}{A} \rho \sigma_{e x} \overline{\epsilon_{e x}}
$$

where $N_{A}$ is Avogadro's number, $A$ is the atomic weight, $\rho$ is the density, $\sigma_{e x}$ is the total excitation cross section (summed over all subshells), and $\overline{\epsilon_{e x}}$ is the mean energy loss due to excitation at a given primary energy. This stopping power is multiplied by the distance between the last event and the present event to get the energy lost to excitations in transit, which is subtracted from the electron's energy before calculating the distance to the next event.

Another option for modeling excitation is to treat it in the same analog manner as the other interactions. There is no deflection angle, and, rather than sampling from a spectrum of possible energy losses, the average energy loss per event (for an electron of the current energy) is used. As can be seen in Table 3 the spectral 
energy loss distributions for individual excitation events is not available at this time.

\subsubsection{Compounds and Mixtures}

A special version of the CREEP code handles all compounds and mixtures by combining the EEDL element data using Bragg additivity of cross sections. This method was originally introduced for determining the stopping power of a compound by computing a weighted sum of the stopping powers of the atomic constituents of the compound, and was recently described in ICRU 37 [47]. To compute the total cross section of a compound, $\sigma$, the additivity rule takes the form

$$
\sigma=\sum_{j} w_{j} \sigma_{j}
$$

where $w_{j}$ is the fraction by weight and $\sigma_{j}$ is the cross section of the $j^{\text {th }}$ atomic constituent.

To use the compound/mixture version of CREEP, the user must enter weight fractions of each element in the compound or mixture, and the density for the compound. This simple approach does not account for any chemical binding effects, which start to become important near the binding energy of the medium, or other effects arising from the chemical state or neighboring molecules in the medium.

The algorithm is the same as that described in the beginning of the previous discussion for elements, except that once the distance to the next interaction is found, the next step is to select which medium the electron will interact with (by comparing a random number to their mass-fraction weighted cross sections) and then selecting the type of interaction as usual within that medium. 


\section{$2.3 \quad$ CREEP Results}

The benchmarking of CREEP results with experimental data for a variety of elements and select compounds and mixtures, over the energy range of the EEDL database, is a large effort that is still in its infancy. There are many possible outputs of this code to be analyzed; some of which can be readily compared to experiments and theoretical models. Other information has no feasible experimental equivalent and as such is of interest primarily in a qualitative sense (such as "event maps" which plot interaction sites for all types of interactions). A few examples of both quantitative and qualitative results are shown in the remainder of this section.

\subsubsection{Backscatter Studies}

Historically, backscatter has been difficult for condensed history codes to simulate correctly. Figures 10 and 11 show two examples of backscatter information generated by CREEP compared to experimental values. The agreement is within less than a percent for the backscatter coefficients. For the backscattered energy spectrum, the binning structure within CREEP was chosen to match that of Darlington [19], and the the agreement with those results is very close: the maximum deviation is approximately $0.2 \%$, with CREEP being consistantly slightly higher than Darlingtion. The results from Kanter [34] show a similar overall shape, but a magnitude that is consistantly $8-10 \%$ higher than CREEP or Darlington.

\subsubsection{Transmission Studies}

Figures 12 and 13 strikingly illustrate both the strengths and the limitations of the present version of CREEP. For each of the four curves shown, an electron of relatively low energy (not more than 500 times the binding energy) is incident 


\section{Comparison of Backscatter Percentages in Thick Al}

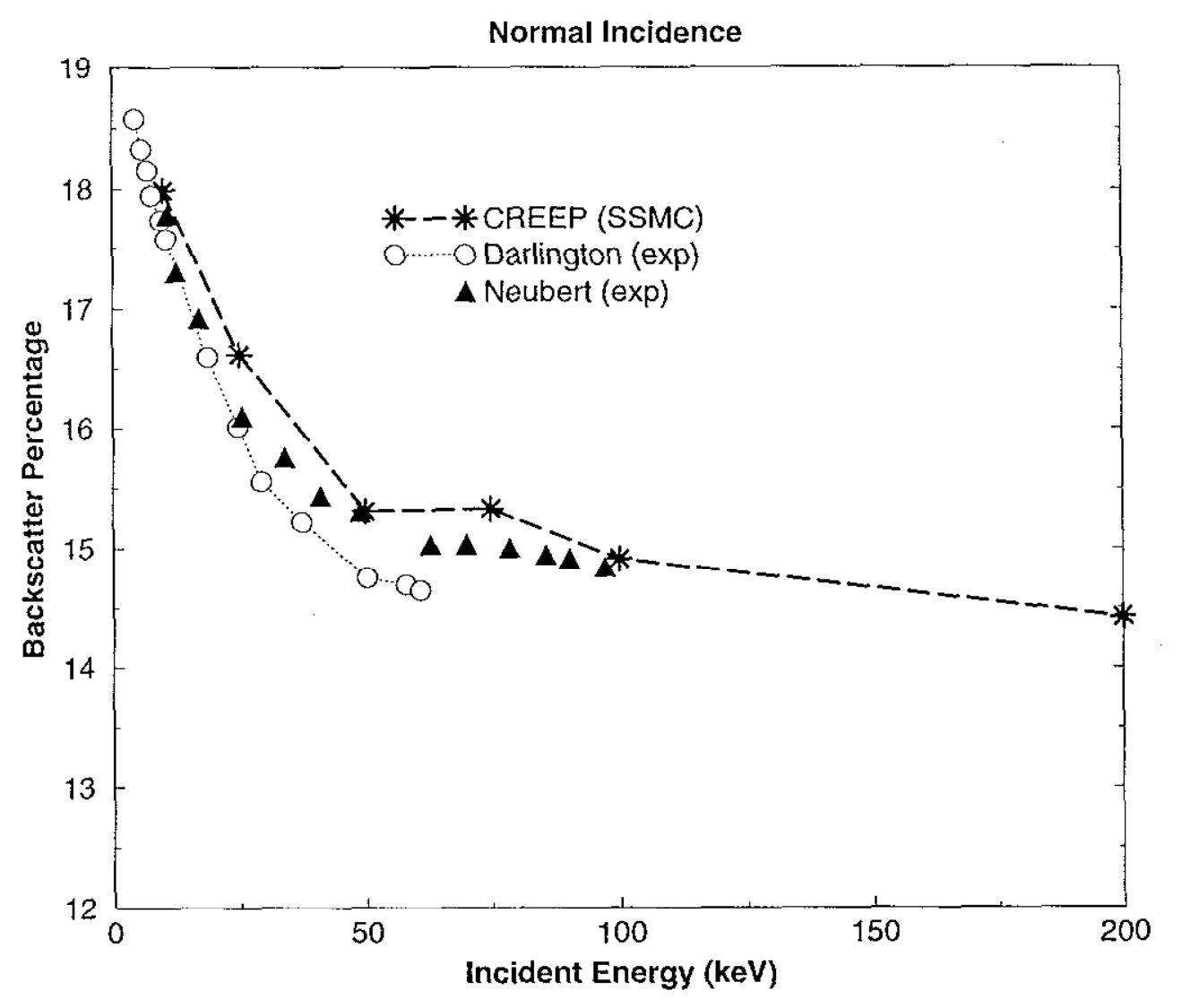

Figure 10: CREEP backscatter percentage (including backscattered secondary electrons) compared to the experiments of Darlington [19] and Neubert [43]. 


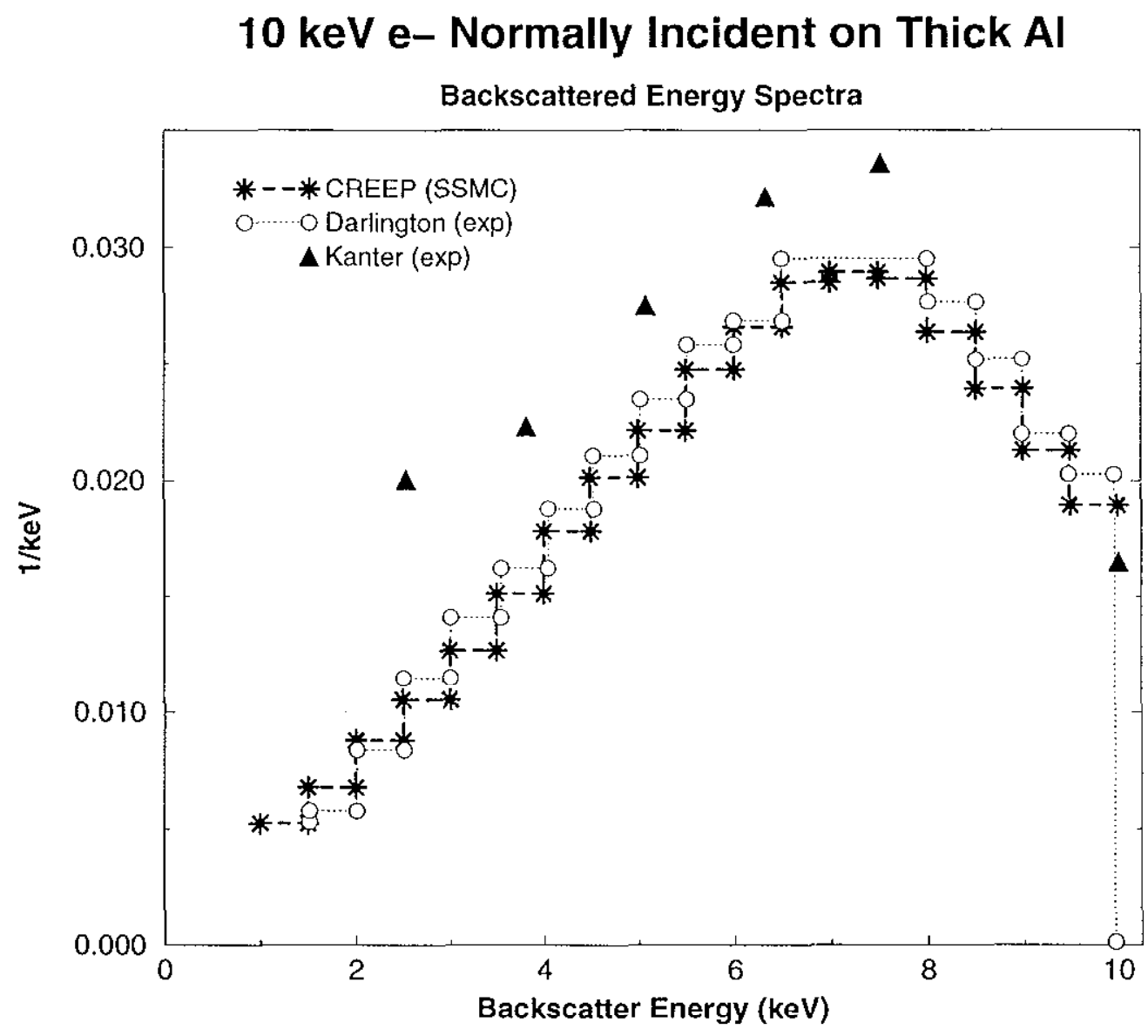

Figure 11: The backscattered energy spectrum resulting from a $10 \mathrm{keV}$ electron impinging on the surface of an aluminum slab that is large in $x, y$, and $z$ compared to the mean free path of the incident electron, compared to experiments of Darlington [19] and Kanter [34]. 


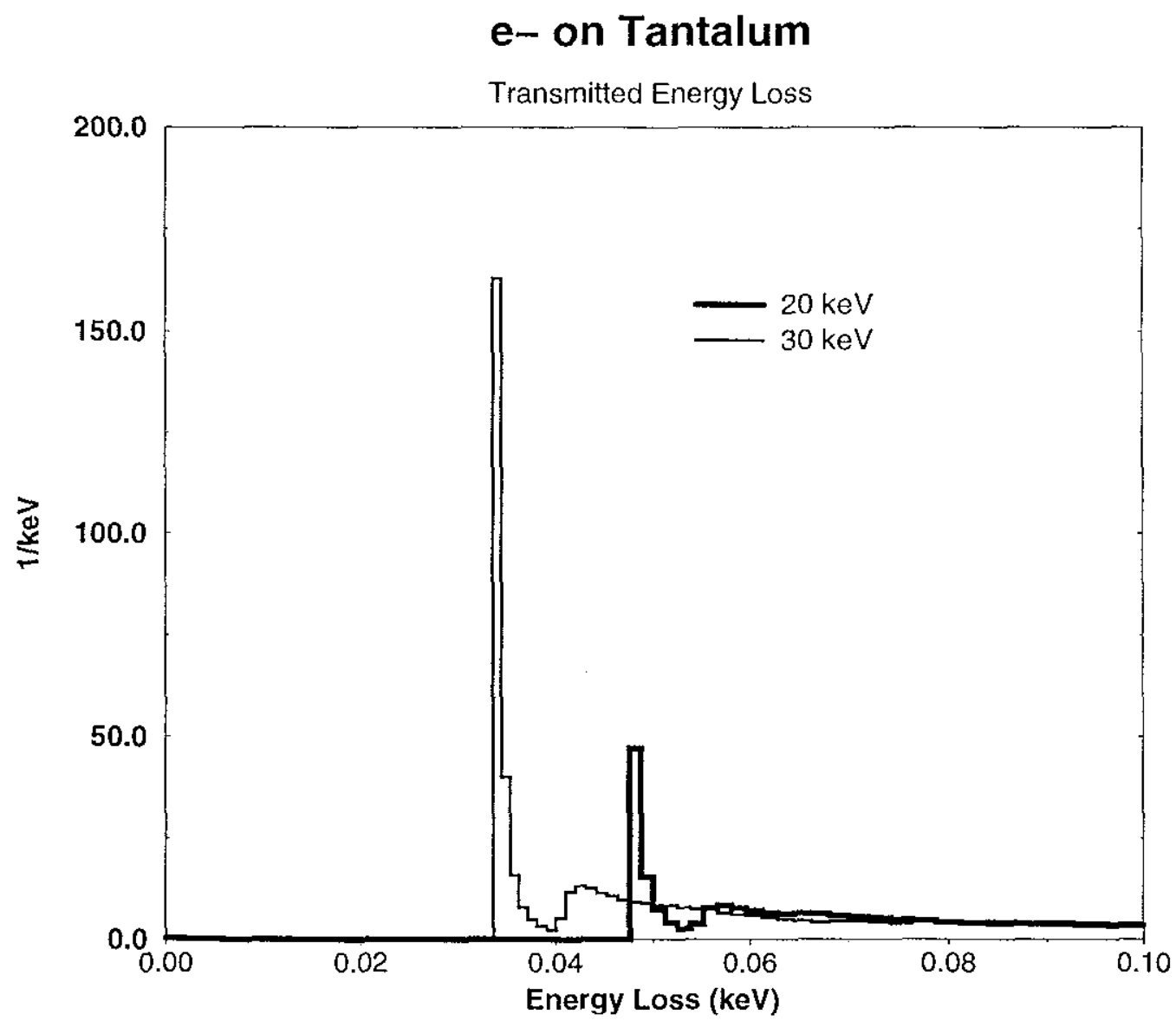

Figure 12: The exiting energy loss spectra after $32 \mathrm{keV}$ electrons are transmitted through gold $(\mathrm{Z}=79)$ slabs of two different thicknesses: $2.87 \times 10^{-6} \mathrm{~cm}$ (approximately 10 mean free paths) and $5.74 \times 10^{-6} \mathrm{~cm}$ (approximately 20 mean free paths). 


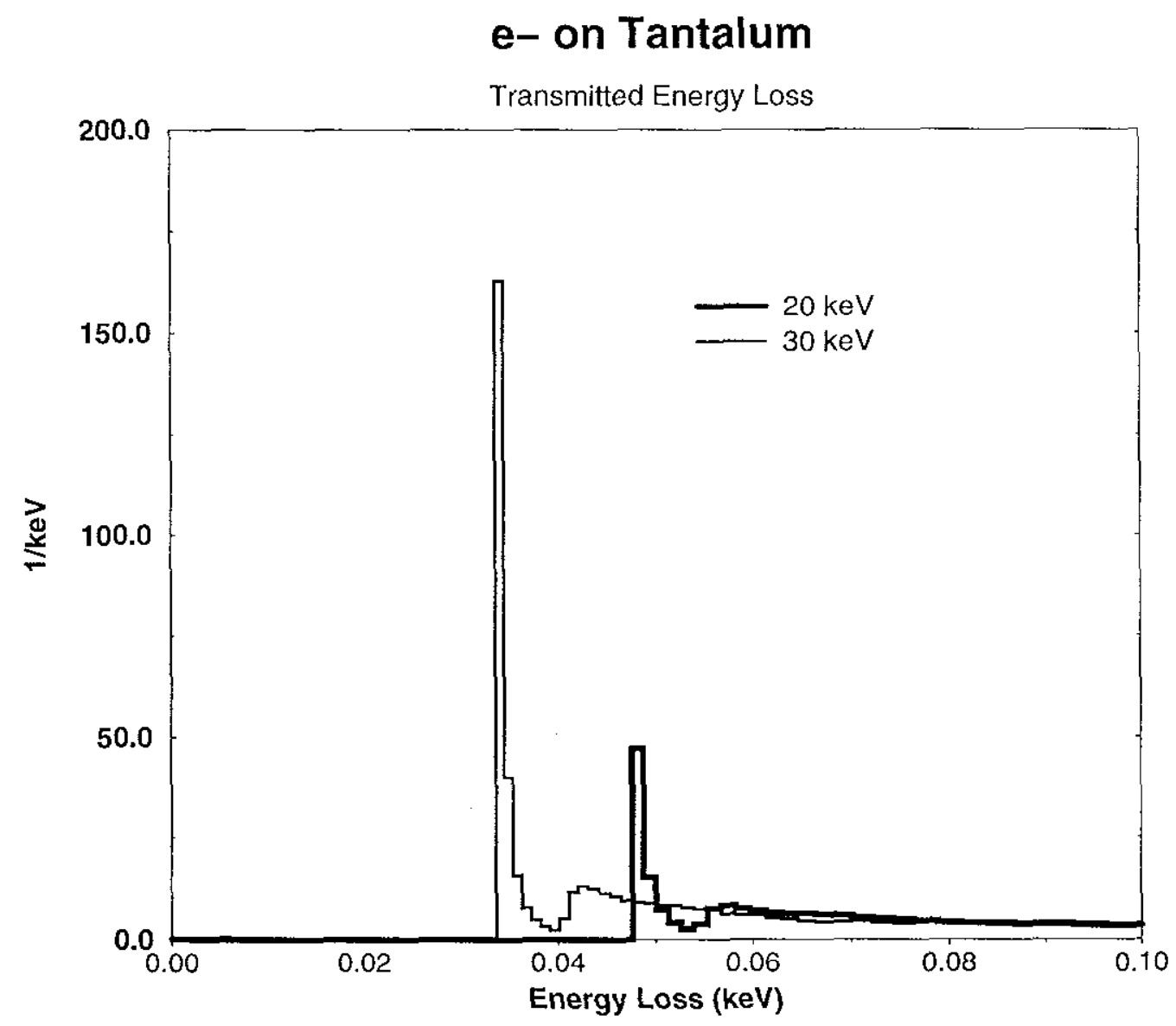

Figure 13: The exiting energy loss spectra after $32 \mathrm{keV}$ electrons are transmitted in tantalum $(\mathrm{Z}=73)$ for a constant thickness of $3.0 \times 10^{-6} \mathrm{~cm}$ at two different energies. See text for discussion. 
on a thin slab (not more than 20 mean free paths) and the amount of energy each electron lost after having traversed the slab is tallied. Traditional energy loss distributions described in Chapter 1, such as the Gaussian having the width given in equation 1.12, would predict a wide, smooth distribution. SSMC gives a highly structured, asymmetric distribution, explained below. Both distributions have mean values equal to the stopping power predicted loss.

The first distinctive feature of the curves in Figures 12 and 13 is a zeroamplitude region in the low energy loss region, implying that no electrons escape without losing at least some energy, due to the continuous nature of the excitation loss model given by equation 2.16. This region ends abruptly at the energy loss that corresponds to the excitation-only stopping power times the thickness of the slab, where a sharp peak is seen. The peaks are due to electrons that escape the slab without undergoing any ionization (or bremsstrahlung) events. The sharpness of these peaks is therefore an artifact resulting from not modeling individual excitation events.

The next distinctive feature of these curves is a gap of low amplitude following the peak. This discontinuity is due to the binding energy of the material, which, for gold $(Z=79)$ is $8.3 \mathrm{eV}$ and for tantalum $(Z=73)$ is $7.31 \mathrm{eV}$. If an electron doesn't escape without ionization, it must give up the binding energy (in this code locally deposited in the medium), which explains the lack of electrons seen until the low, broad peak. The shoulder on the large-energy-loss side of this peak rolls off very gradually, as there is progressively smaller probability of multiple ionization events and/or single large energy loss events.

\subsubsection{Energy Deposition Curves}

Comparisons of the CREEP single scatter Monte Carlo (SSMC) code with experiment are shown in Figure 14 - Figure 20. The points attributed to Lockwood et al 
are from calorimetric measurement [38]; the comparisons are absolute. Depths are reported as a fraction of the CSDA range of the electron, which were taken from ICRU Report 37 [47].

When assessing CREEP's agreement to each of these calorimetry curves, it is important to bear in mind the thickness of the calorimeter, which is given in the legend for each figure. At low energies, this thickness can represent as much as nearly $30 \%$ of the range of the the electron, such that the resolution of the energy deposition is "blurred" by the width of the detector. An example of this can be seen in the results for $100 \mathrm{keV}$ electrons in beryllium shown in Figure 17. The calorimeter width is $28 \%$ of the mean range, causing the measurements to "flatten" the peak of the curve and spread out the taper at the end of the curve. The results for $300 \mathrm{keV}$ and $500 \mathrm{keV}$, where the calorimeter thickness is a much smaller fraction of the mean range ( $5 \%$ and $2 \%$, respectively) appear to be in much closer agreement to CREEP.

Overall, these benchmarks show the code is performing well in the areas tested. Agreement to experiment is generally within $3-5 \%$ for a variety of materials, incident energies, and incident angles. 


\section{Energy Deposition vs Depth in Aluminum}

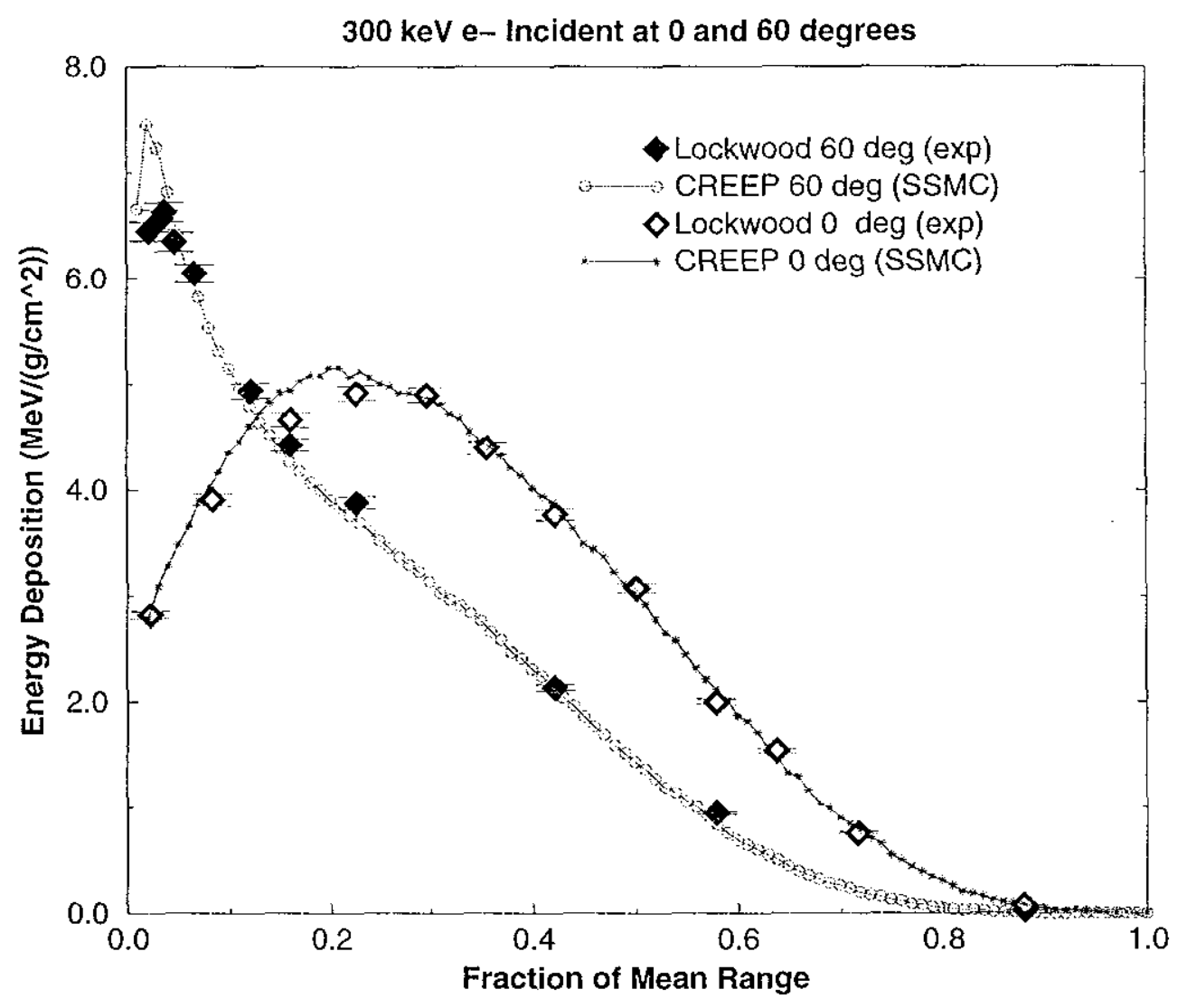

Figure 14: Energy deposition is shown as a function of depth into the medium. The depth has been normalized to $R_{C S D A}=0.1083 \mathrm{~g} / \mathrm{cm}^{2}$ at $300 \mathrm{keV}$ in aluminum. The calorimeter thickness was $5.05 \times 10^{-3} \mathrm{~g} / \mathrm{cm}^{2}=0.0466 \cdot R_{C S D A}$. 


\section{Energy Deposition vs Depth in Molybdenum}

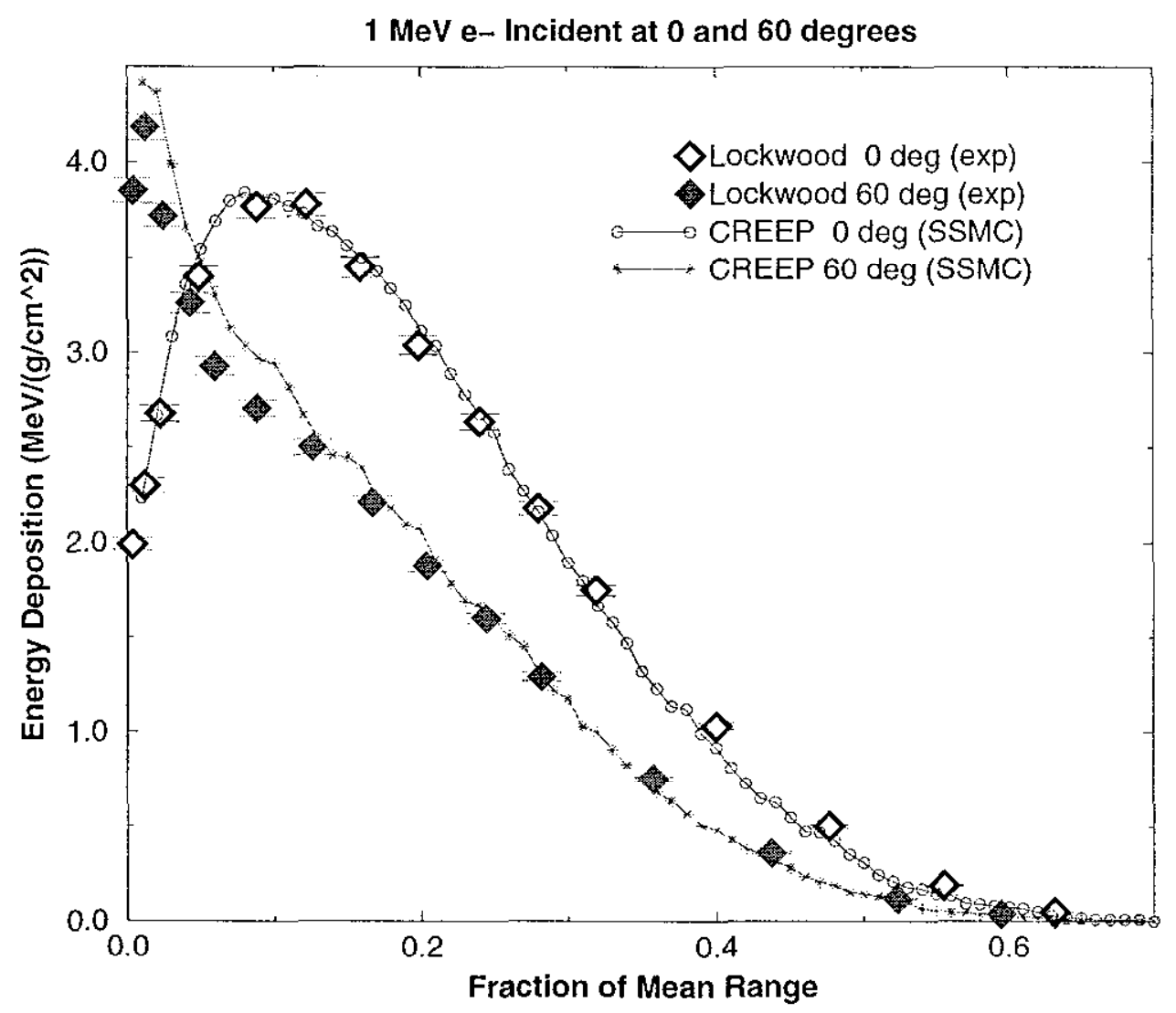

Figure 15: Energy deposition is shown as a function of depth into the medium. The depth has been normalized to $R_{C S D A}=0.6748 \mathrm{~g} / \mathrm{cm}^{2}$ at $1 \mathrm{MeV}$ in molybdenum. The calorimeter thickness was $5.301 \times 10^{-3} \mathrm{~g} / \mathrm{cm}^{2}=0.0079 \cdot R_{C S D A}$. 


\section{Energy vs Depth in Tantalum}

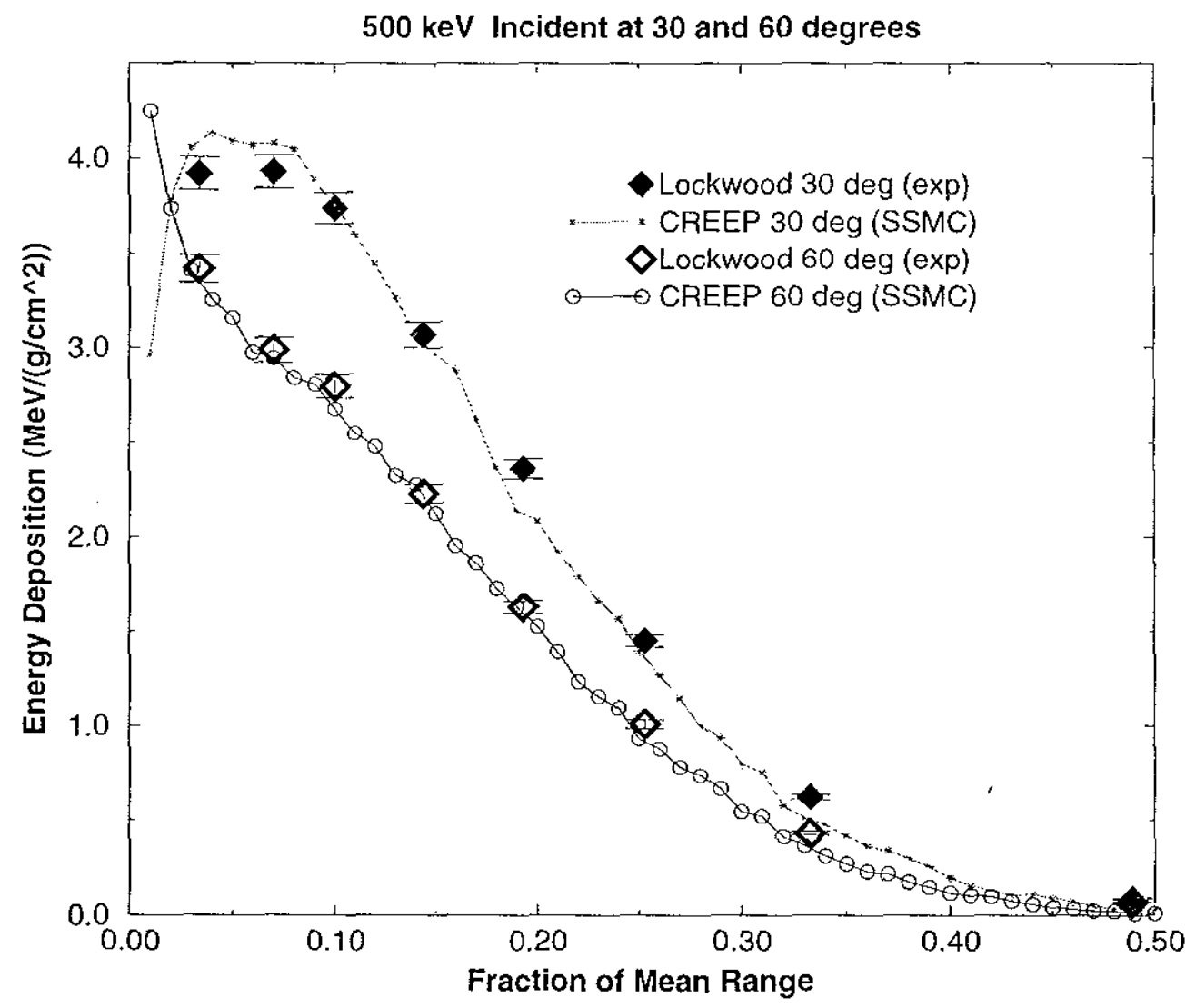

Figure 16: Energy deposition is shown as a function of depth into the medium. The depth has been normalized to $R_{C S D A}=0.3247 \mathrm{~g} / \mathrm{cm}^{2}$ at $500 \mathrm{keV}$ in tantalum. The calorimeter thickness was $1.655 \times 10^{-2} \mathrm{~g} / \mathrm{cm}^{2}=0.0510 \cdot R_{C S D A}$. 


\section{Energy Deposition vs Depth in Beryllium}

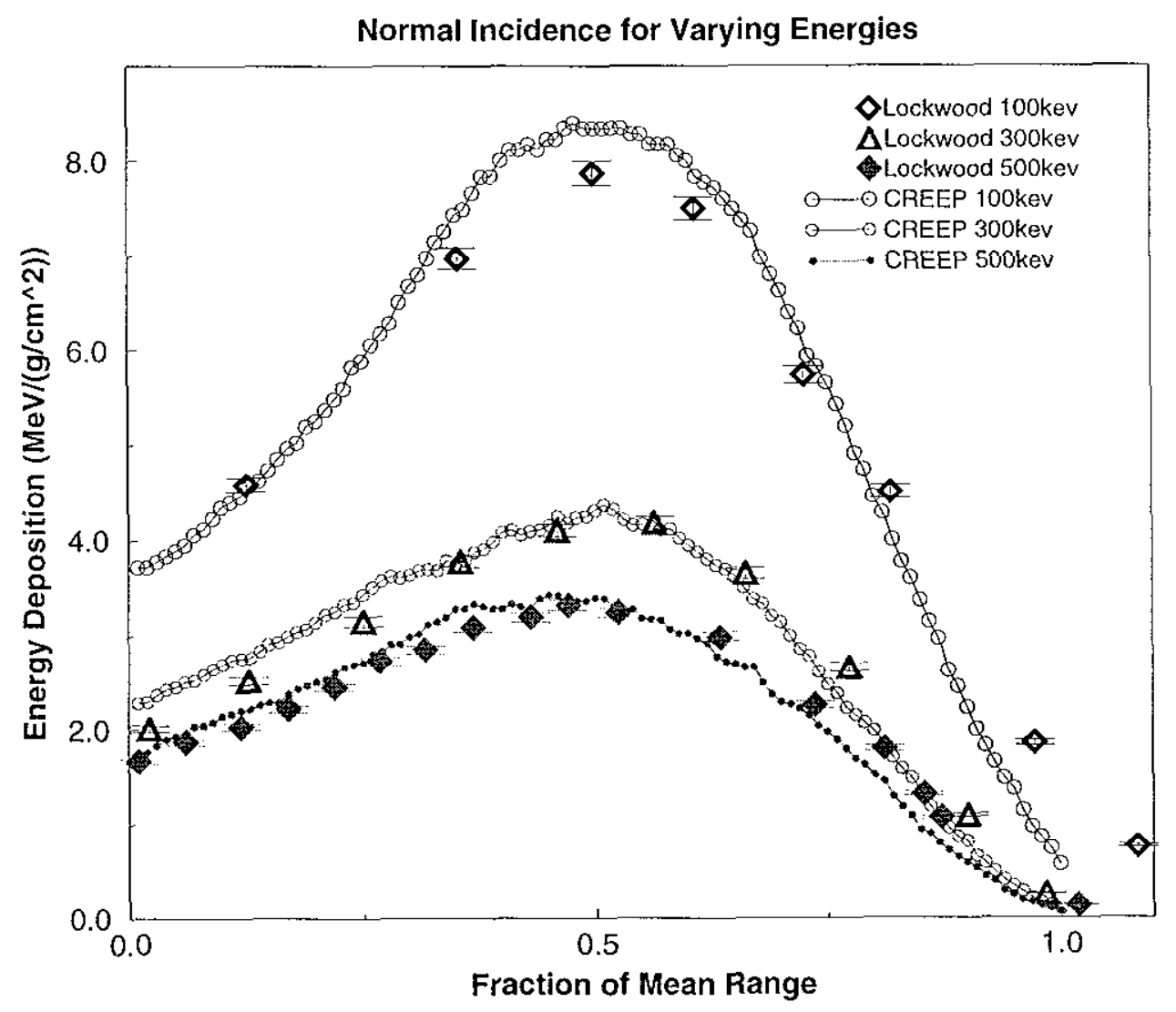

Figure 17: Energy deposition is shown as a function of depth into the medium, where the depth has been normalized to $R_{C S D A}=0.0175 \mathrm{~g} / \mathrm{cm}^{2}, 0.1037 \mathrm{~g} / \mathrm{cm}^{2}$, and $0.2188 \mathrm{~g} / \mathrm{cm}^{2}$ for 100,300 , and $500 \mathrm{keV}$, respectively. The calorimeter thickness was $4.871 \times 10^{-3} \mathrm{~g} / \mathrm{cm}^{2}$. This represents $0.2785 \cdot R_{C S D A}$ at $100 \mathrm{keV}, 0.0470 \cdot R_{C S D A}$ at $300 \mathrm{keV}$, and $0.0223 \cdot R_{C S D A}$ at $500 \mathrm{keV}$. 


\section{Energy Deposition vs Depth in Copper}

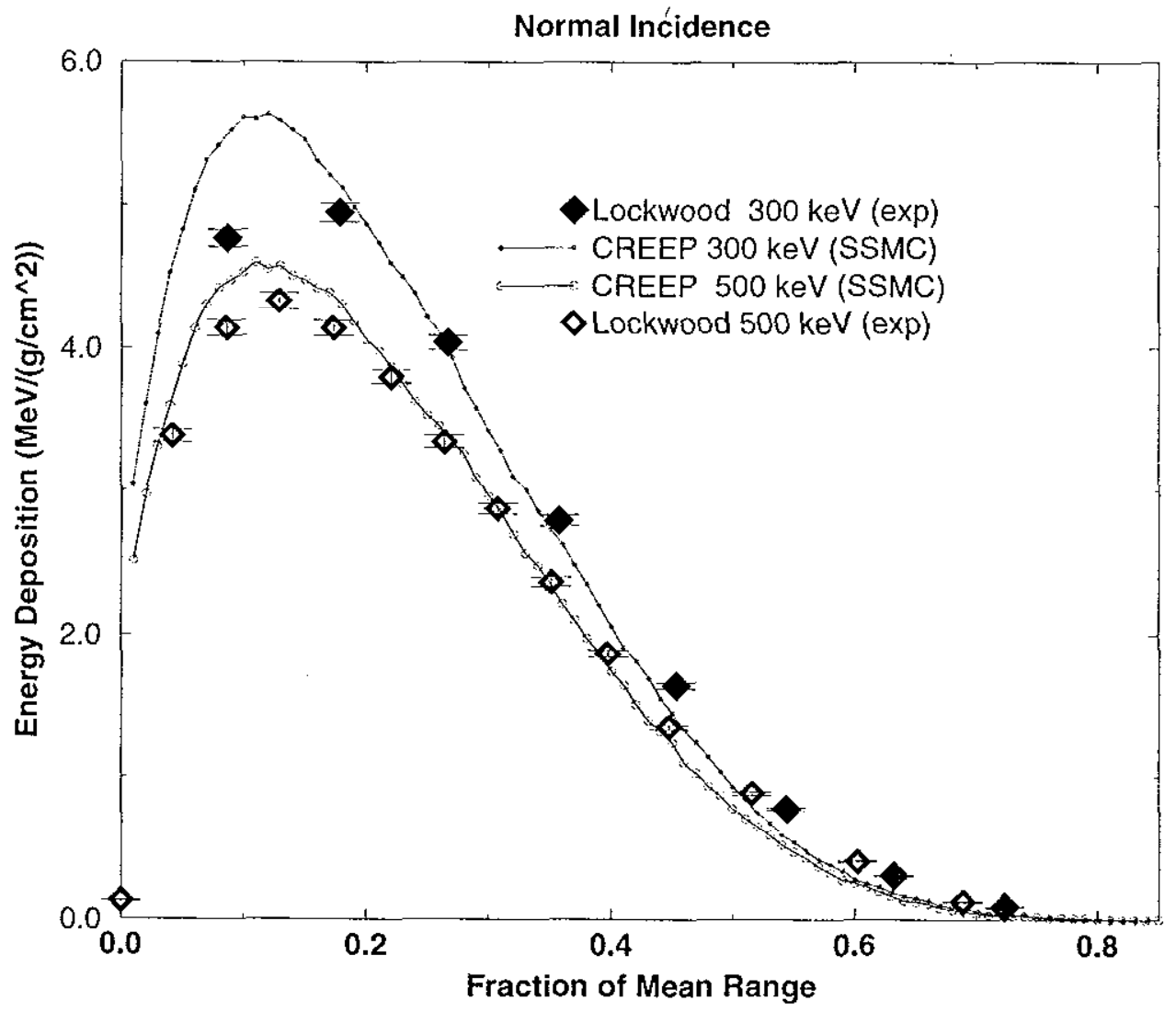

Figure 18: Energy deposition is shown as a function of depth into the medium, where the depth has been normalized to $R_{C S D A}=0.1263 \mathrm{~g} / \mathrm{cm}^{2}$ and $0.2617 \mathrm{~g} / \mathrm{cm}^{2}$ for $300 \mathrm{keV}$ and $500 \mathrm{keV}$, respectively. The calorimeter thickness was $2.194 \mathrm{x} 10^{-2}$ $\mathrm{g} / \mathrm{cm}^{2}$. This represents $0.1737 \cdot R_{C S D A}$ at $300 \mathrm{keV}$, and $0.0838 \cdot R_{C S D A}$ at $500 \mathrm{keV}$. 


\section{Energy Deposition vs Depth in Iron}

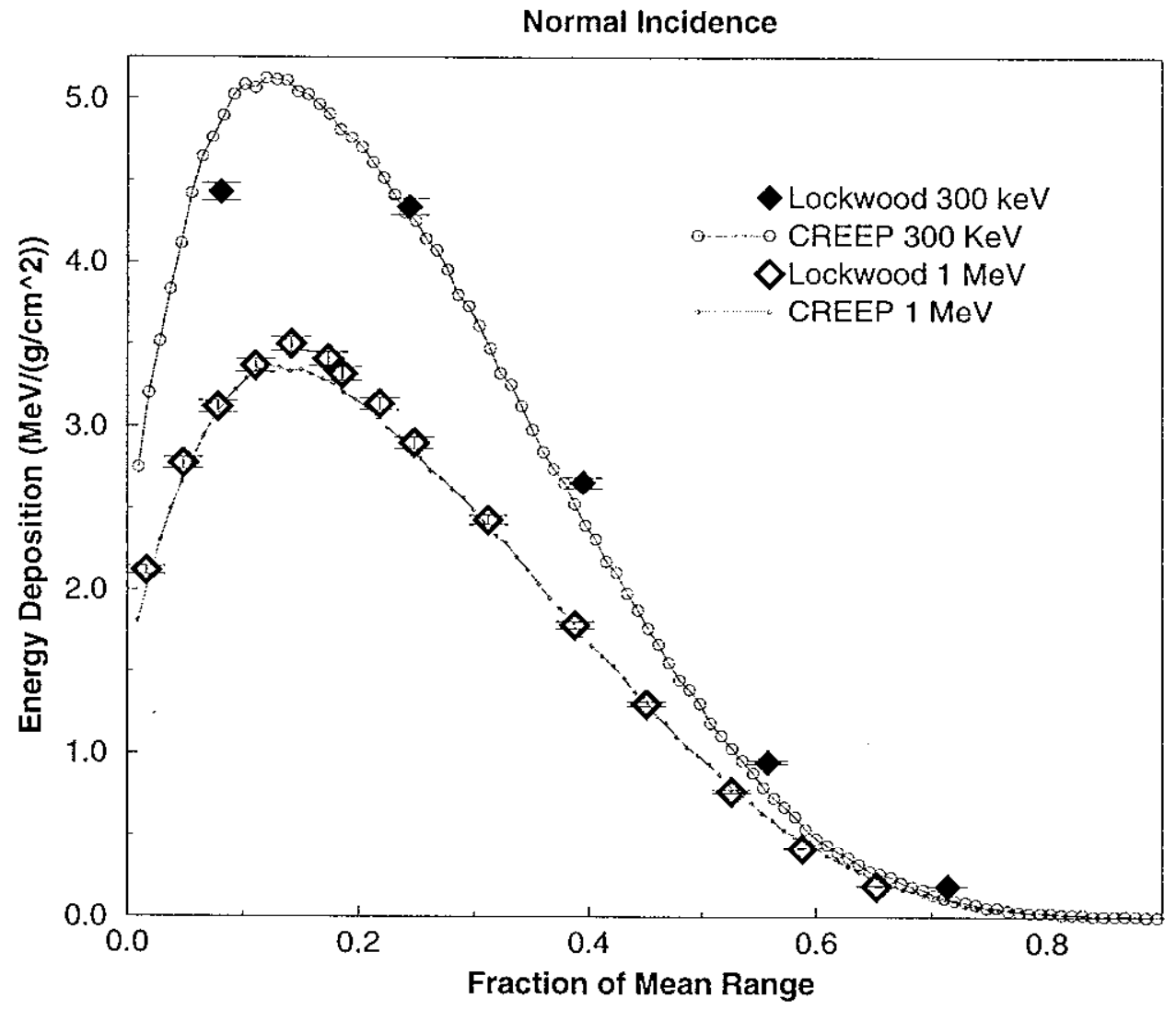

Figure 19: Energy deposition is shown as a function of depth into the medium, where the depth has been normalized to $R_{C S D A}=0.1218 \mathrm{~g} / \mathrm{cm}^{2}$ for $300 \mathrm{keV}$ and $0.6159 \mathrm{~g} / \mathrm{cm}^{2}$ for $1 \mathrm{MeV}$. The calorimeter thickness was $1.956 \times 10^{-2} \mathrm{~g} / \mathrm{cm}^{2}$. This represents $0.1606 \cdot R_{C S D A}$ at $300 \mathrm{keV}$, and $0.0318 \cdot R_{C S D A}$ at $1 \mathrm{MeV}$. 


\section{Energy Deposition vs Depth in Carbon}

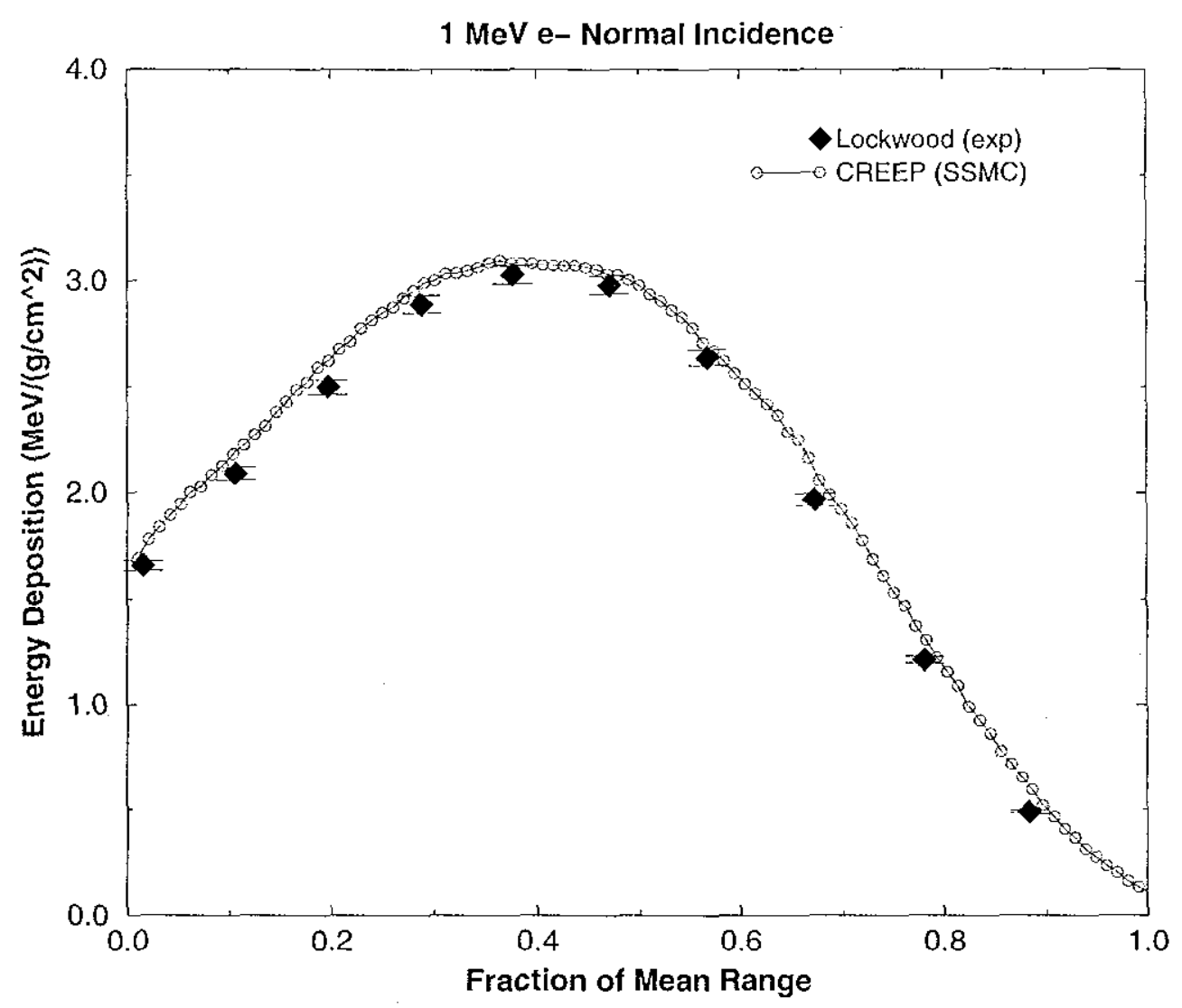

Figure 20: Energy deposition is shown as a function of depth into the medium. The depth has been normalized to $R_{C S D A}=0.4981 \mathrm{~g} / \mathrm{cm}^{2}$ for carbon at $1 \mathrm{MeV}$. The calorimeter thickness was $1.561 \times 10^{-2} \mathrm{~g} / \mathrm{cm}^{2}$, which is $0.0313 \cdot R_{C S D A}$. 


\subsubsection{Other Quantities of Interest}

In addition to the preceding quantities, CREEP also calculates analog stopping powers (the amount of energy lost per unit distance for both radiative and collisional events), energy deposits due to individual interaction types, and "real" pathlength (cumulative distance between events) that can be used to calculate detour factors, which are the ratio to the real range compared to the CSDA range.

\subsection{Discussion}

Single scatter electron transport is time consuming. This method is not intended to be a general-purpose means of electron transport, but rather a powerful tool for use in situations where it is desirable to obtain information about the basic interaction of electrons with the medium. Some timings are shown in Table 4. In general, the simulation time increases with the number of histories, the geometry size, and as the energy threshold is lowered. All of these require more interactions to be simulated. The version of the code that includes compounds and mixtures is also notably slower than the element versions, due to the need to find cross sections in each element for every step, and then compare them to decide in which element the interaction will take place. Table 4 gives some feel for how the run time scales with different media.

Clearly if this method is to become practical, there must be a means for a radical speed up in the execution time. Such a means has been suggested in the Local-to-Global algorithm [56]. The Local-To-Global concept relies on tallying pre-computed probability distributions resulting from a geometry element of fixed size, for a fixed incident energy and material type. The remaining chapters of this thesis will discuss using CREEP as the local stage of the Local-to-Global MRMC method. 
Table 4: Timings for several CREEP runs on a SunSparc 20 running Solaris OS 2.51. Each medium was a slab of $1 \mathrm{~mm}$ thick. Results for the number of interactions, the number of calls to the random number generator (RNG), and the user time are normalized per incident history.

\begin{tabular}{c|c|c|c|c|c} 
Medium & Density $(\mathrm{g} / \mathrm{cc})$ & Interactions & RNG calls & User Time (s) & Ratio \\
\hline \hline $\mathrm{H}$ & $1 \times 10^{-4}$ & 0.667 & 8 & $2.52 \times 10^{-4}$ & 1 \\
\hline $\mathrm{O}$ & $1.4 \times 10^{-3}$ & 5.098 & 39 & $1.77 \times 10^{-3}$ & 7.02 \\
\hline $\mathrm{Na}$ & 1.0 & 6714 & 48001 & 1.35 & 5357 \\
\hline $\mathrm{H}_{2} \mathrm{O}$ & 1.0 & 8057 & 71714 & 3.30 & 13095 \\
\hline $\mathrm{Au}$ & 19.3 & 19810 & 145541 & 6.98 & 27698 \\
\hline
\end{tabular}

\subsection{Summary of Single Scatter}

The need for a reliable low-energy electron transport method is currently great, and continues to grow with advances in microdosimetry and microtechnology, especially given the fact that some condensed history approximations tend to deteriorate at low energies and/or small step sizes [4].

Can SSMC fill this need? The answer of course depends on the context. Although there are no inherent step size limitations, at energies comparable to the binding energy of the material, there are at least three approximations in CREEP that become weak, as pointed out earlier. First, the kinematics which are used to find the scatter angle after an ionization event neglect binding energy. This alone will probably not cause a major perturbation in the electron trajectory, since large-angle electron scatter is primarily an elastic event. Second, in compounds, chemical binding effects are neglected. So, for example, the binding energy of an $\mathrm{H}_{2}$ molecule is $16.4 \mathrm{eV}$, but CREEP would use the binding energy of elemental hydrogen, $13.6 \mathrm{eV}$. Third, as mentioned previously, the binding energy is locally 
deposited, rather than simulating the atomic relaxation process and tracking the resultant Auger electrons and fluorescent photons. Although LLNL maintains a database that would address these issues (EADL), it has not been coupled to CREEP's transport. In addition, the non-spectral energy loss treatment of excitation events may also present problems at these energies or higher regimes. A simple energy straggling algorithm for excitation would probably reduce these effects, however. Finally, and most fundamentally, there is the problem of the uncertainty of the cross section data itself. These limits have yet to be fully explored.

Because of its slow run times, the single scatter algorithm presented here is not a general-purpose transport tool. However, there are certain instances in which it is desirable to obtain the most accurate answer possible - regardless of the time required. When does single scatter simulation have the most acceptable run times? It is most feasible when the total number of interactions is relatively small, as in a case involving low initial energy, a thin target, minute volume, or sparse media. Chemically simple media are much faster than media which have many elemental components.

Eventually it is expected that SSMC may become useful as a benchmarking tool for new scattering theories, a learning tool for developing physical intuition about parts of a large problem, and a modeling tool for small instruments such as some ion chambers, and other devices for studying energy deposition or interaction sites on a small scale. 


\section{Chapter 3}

\section{The Macro Response Library}

\subsection{Introduction}

The main purpose for developing the single scatter code, CREEP, introduced in the previous chapter, was to provide data that could be used in a fast, accurate global stepping code, which will be detailed in the next chapter. The crucial bridge between these two codes is the library that holds all the single scatter results. An overview of the library is shown in Figure 21. This chapter will describe the contents of the MRMC data library, with emphasis on the various quantities that comprise a single kugel data set. There is one kugel data set for each single scatter run of a given material, size, and incident energy.

Recall that CREEP transports all primary and secondary electrons from the center of the sphere, until they either 1) fall below the energy threshold (meaning they are absorbed inside the kugel), or 2) escape through the surface of the kugel. All escaping electrons have their exit conditions tallied in a variety of different histograms so that the resulting distributions can be normalized, reformatted and stored for sampling in the global transport code.

Separate tallies are kept for primary electrons and knock-on electrons. Photons are also tallied separately. Since CREEP does not track photons, their information is projected to the surface of the kugel along their birth trajectory. They are then tallied in the same routine as the electron data. Any photon interactions that may have occurred between the birth point and the kugel radius are lost in this 


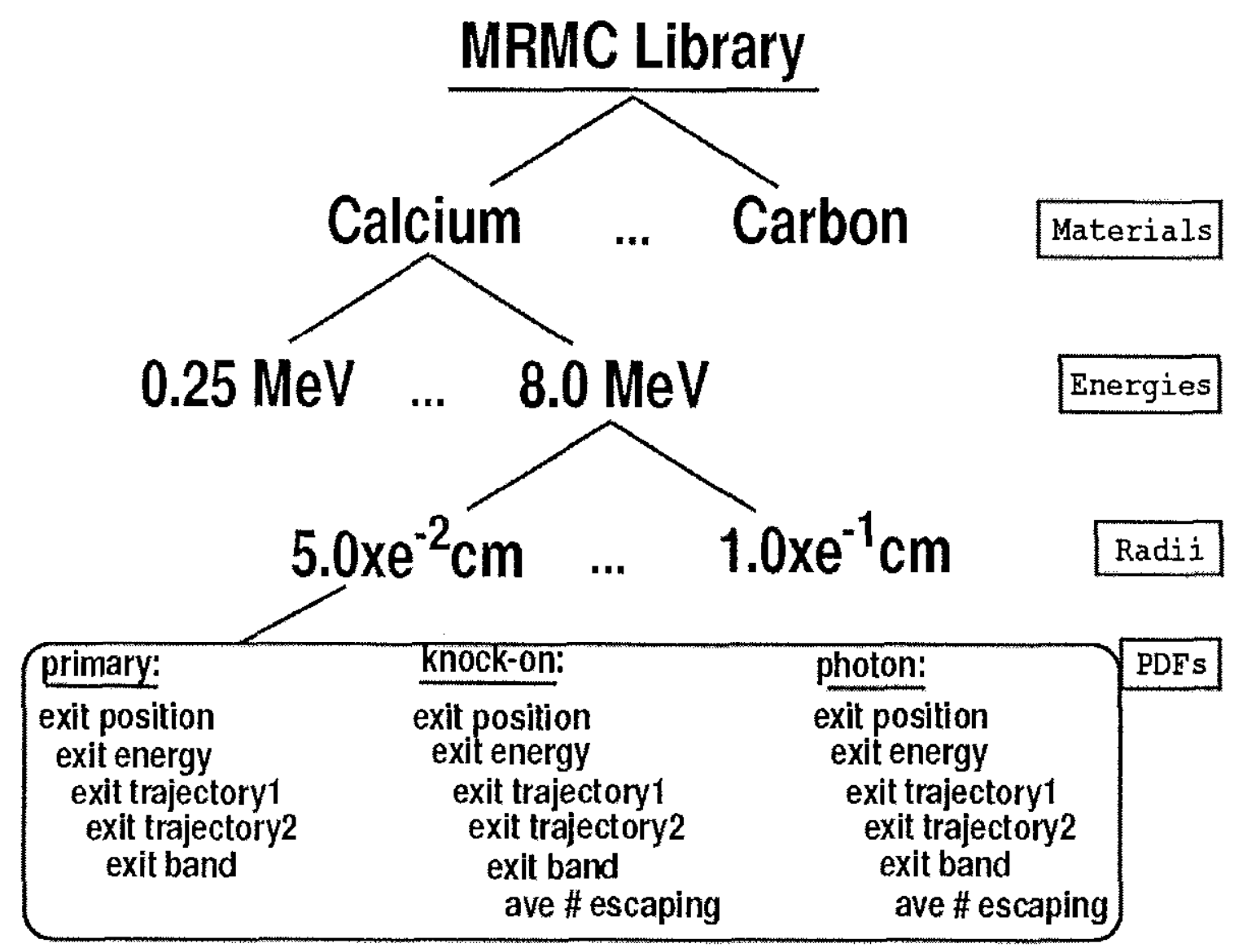

Figure 21: A partial schematic of the MRMC library. Each level in the tree represents a new directory. Only two possible entries are shown at each level for brevity. The probability distribution functions (PDFs) at the lowest level comprise a complete kugel data set. 
scheme, but the kugel radii are typically much smaller than the mean free path of the photon.

\subsection{Correlation of Phase Space by Exit Bands}

Electron phase space variables are highly correlated to one another. An ideal database would have an independent set of distributions for trajectory, position and other state variables at each possible exit energy (for example), because the other variables are all effectively functions of energy. For instance, one can imagine the electrons that pass through the kugel losing only the amount of energy due to excitation events do not undergo much angular deflection, which means their exit points are tightly clustered about the kugels' z-axis, and they have not created many energetic secondaries. In contrast, the electrons that did give up a great deal of energy also scattered through relatively wide angles (resulting in a greater dispersion of location at the kugel surface) and were more likely to have set energetic knock-ons and photons in motion.

Since having a separate set of data for each exit energy would result in prohibitive memory problems, a simpler approach is taken. The phase space is first partitioned by exit location, that is, each kugel surface is divided into bands. The bands on the kugel surface can be considered analogous to latitude lines on the earth, where the kugel's z-axis corresponds to the earth's axis of rotation. All phase space tallies are kept separate for each band, so that only electrons having approximately similar phase space are lumped together in distributions. ${ }^{1}$

The existing library was compiled using four bands per kugel for electrons, with variable spacing along the z-axis, as shown in Figure 22. The number four was chosen as a compromise between several factors. A minimal number was desired

\footnotetext{
${ }^{1}$ Photon phase space is tallied into a single set of distributions - the entire kugel surface is one band to photons.
} 
because the amount of memory required for the MRMC library increases as a multiple of the number of bands. Furthermore, the statistics in the local PDFs become worse as the number of bands increases (particularly as the low-probability backscatter region is split), or conversely, for the run time for statistics equivalent to the single-surface band case increases. On the other hand, the aforementioned correlation is tighter, and the accuracy of the MRMC method as a whole is, in principle, improved with a large number of bands.

Previous incarnations of the Local-to-Global method by Neuenschwander, described in Section 1.6.1, and Ballinger, described in Section 1.6.2, used 1 and 8 ( 5 forward +3 backscatter) surface bands, respectively. Since the intended application for this code was most similar to Neuenschwander's successful MMC code, four bands was considered likely to be adequate. Indeed when a fifth band was added in the backscatter region of the kugel shown in Figure 22, the PDFs resulting from the local calculation were not different enough to justify the extra time and memory associated with their generation.

Use of the band structure is straightforward. When an electron exits the kugel, its $z$-coordinate is normalized to the radius of the kugel to obtain its position cosine, $z_{k u g} / R_{k u g}$. This position cosine is compared to the band boundaries to determine from which exit band the electron escaped. Additional details will be discussed in Section 3.4.2.

\subsection{CREEP Kugel Phase Space Tallies}

For the sake of simplicity, all tallies discussed in this chapter have 100 equally spaced bins, which is a somewhat arbitrary assignment, but easy to manage. Ideally, the number and spacing of bins would be optimized for each quantity (as would the number and spacing of bands), for each incident energy, and for each material. Optimization of the resolution in these tallies is an area for future research. 


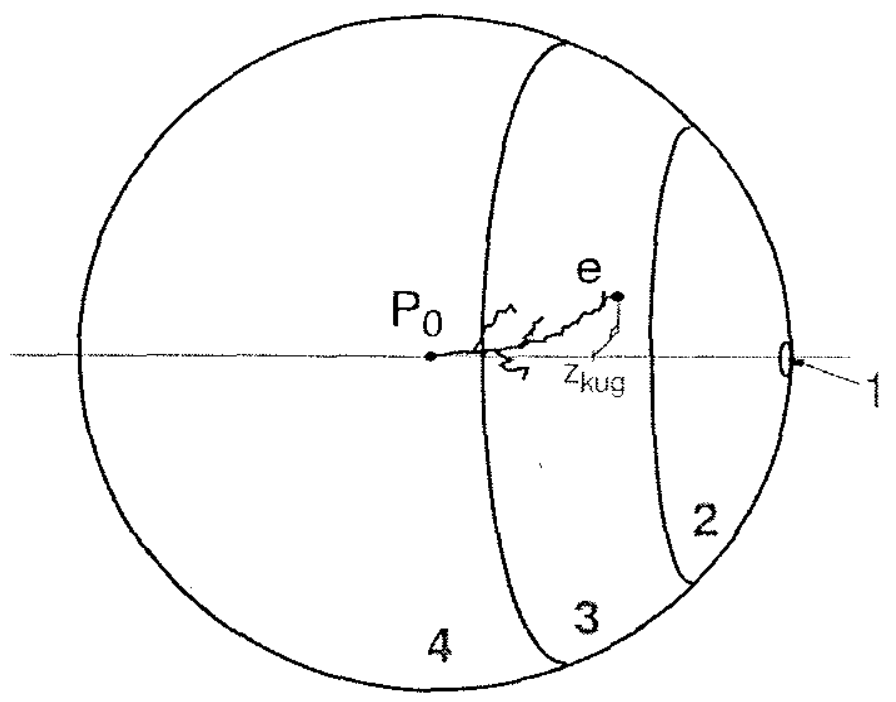

Figure 22: A diagram of the kugel geometry showing the four surface exit bands. All electrons are incident at $P_{0}$, the center of the kugel. The electron track shown has several knock-ons and escapes at the sampled point $e=\left(x_{k u g}, y_{k u g}, z_{k u g}\right)$ which is in Band 3. The bands are spaced closely in the forward direction due to the forward peaked nature of the electron distributions. 
There are a minimum of 44 separate 100-bin tallies for each kugel library entry: 4 quantities ( $\mathrm{x} 4$ bands) for primary electrons, 5 ( $\mathrm{x} 4$ bands) for secondary electrons, and $4(\mathrm{x} 1$ band $)+1$ ( $\mathrm{x} 4$ bands) for photons; as well as 4 -bin band probabilities for each of the 3 particle types, and a few other quantities that must be stored. (Memory requirements for this information is discussed in Section 3.7.) Additional details for each of the tallies are given below.

\subsubsection{Primary Position Cosine}

This quantity, $z / R_{\text {kug }}$, has variable minimum and maximum values depending on the extrema defining the band. For the first band, the position cosines cover the range from unity to the minimum limit on band 1 (0.98), the second band has 100 bins between band 1 min and band 2 min, etc. Because the limits on each band are known by definition (and since the number of bins per distribution is constant), this is the most finely resolved quantity in the database; there are 400 bins across the surface of the kugel.

\subsubsection{Primary Exit Energy}

This quantity, $E_{\text {out }}$, is tallied as the fraction of the incident energy, $E_{\text {out }} / E_{\text {in }}$, from 0 to 1. By normalizing to the incident energy before the value is stored, a few operations are saved in the global calculation, since it is the fraction which must be multiplied by the real electron energy (which is usually not the same as the energy at which the distributions are tallied) to determine the energy for the next global step. In addition, it is more revealing to plot the normalized energy distributions of various incident energies together, as can be seen in Figures 48-51. The flat region at the high end of each curve with 0 -amplitude is due to the energy that must be given up to traverse the radius of the kugel. Note lower energy electrons must give up a larger fraction of their energy to cross the kugel. 
Other methods of tallying the exit energy have been suggested [40]. For instance, the quantity could be tallied as

$$
w=\frac{E_{\text {out }}+\frac{d E}{\rho d x} \cdot \rho R_{k u g}}{E_{\text {in }}}
$$

where $\frac{d E}{\rho d x}$ is the mass stopping power, such that the term $\frac{d E}{\rho d x} \cdot \rho R_{k u g}$ represents the expected energy loss. This has the advantage that it would scale very well from the tabulated energies to the actual energy of the kugel because it varies slowly with energy. However, it would require a slightly more complicated binning structure than the simplistic version presented here, since the limits of $w$ depend on the incident energy. For example, at $0.25 \mathrm{MeV}$ in a $0.5 \mathrm{~cm}$ radius calcium kugel, the bins would have extrema of

$$
\begin{aligned}
& w_{l o}=\frac{\frac{d E}{\rho d x} \cdot \rho R_{k u g}}{E_{\text {in }}} \approx 0.65 \\
& w_{h i}=1+\frac{\frac{d E}{\rho d x} \cdot \rho R_{k u g}}{E_{\text {in }}} \approx 1.65,
\end{aligned}
$$

while a kugel initiated with an energy of $8.0 \mathrm{MeV}$ would have values approximately of 0.02 , and 1.02 , respectively. The distributions resulting from this method are slightly less intuitive, which is a factor in the developmental stages of a code, but it is probably a better choice for future use because the dynamic range of the stored value is stretched.

\subsubsection{Primary Exit Trajectory}

There are two main challenges to consider when tallying the electron's exit trajectory from the kugel. First, an appropriate frame of reference which takes advantage 
of the symmetry of the problem must be chosen. Second, a way of preserving the relative importance of each component of the trajectory must be devised.

\section{Reference Frame for the Trajectory Tally}

If the electron did not scatter as it traversed the kugel, it would emerge normal to the surface. Thus, measuring the components of the exit trajectory with respect to the normal is an intuitive and sensible approach. The normal frame is translated by $\overrightarrow{R_{0}}=\frac{x_{k u g}}{R_{k u g}} \hat{x}+\frac{y_{k u g}}{R_{k u g}} \hat{y}+\frac{z_{k u g}}{R_{k u g}} \hat{z}$, and rotated by an angle $\theta$ compared to the kugel reference frame. It is defined by the surface normal, $\vec{n}$, at the exit point, $e$, as the vector relative to which the trajectory is tallied; the axis along $\vec{o}$, which is defined as the vector which is normal to $\vec{n}$ and intersects the kugel's z-axis at point $z_{0}$, which is a distance $t$ from $e$; and the axis along $\vec{p}$, which defined by

$$
\vec{p}=\vec{n} \times \vec{o}
$$

This geometry is illustrated in Figure 23.

The first attempt to tally direction cosines simply translated the kugel coordinate system to the sampled exit point and did not rotate. The result was that the distributions were flattened out, because, although most electrons were emerging approximately in the normal direction, each exit location had a different representation of the "normal" vector. The flattened distribution meant that the global sampling algorithm (discussed in the next chapter) was unlikely to choose a exit trajectory that corresponded to the normal vector at the sampled exit point.

In order to tally the exit vector, $\vec{R}_{1}$, with respect to the normal frame, the components of the normal frame axes must be expressed in terms of the kugel frame coordinates. The components of $\vec{n}$ depend on the exit point, $e$, and are given by 

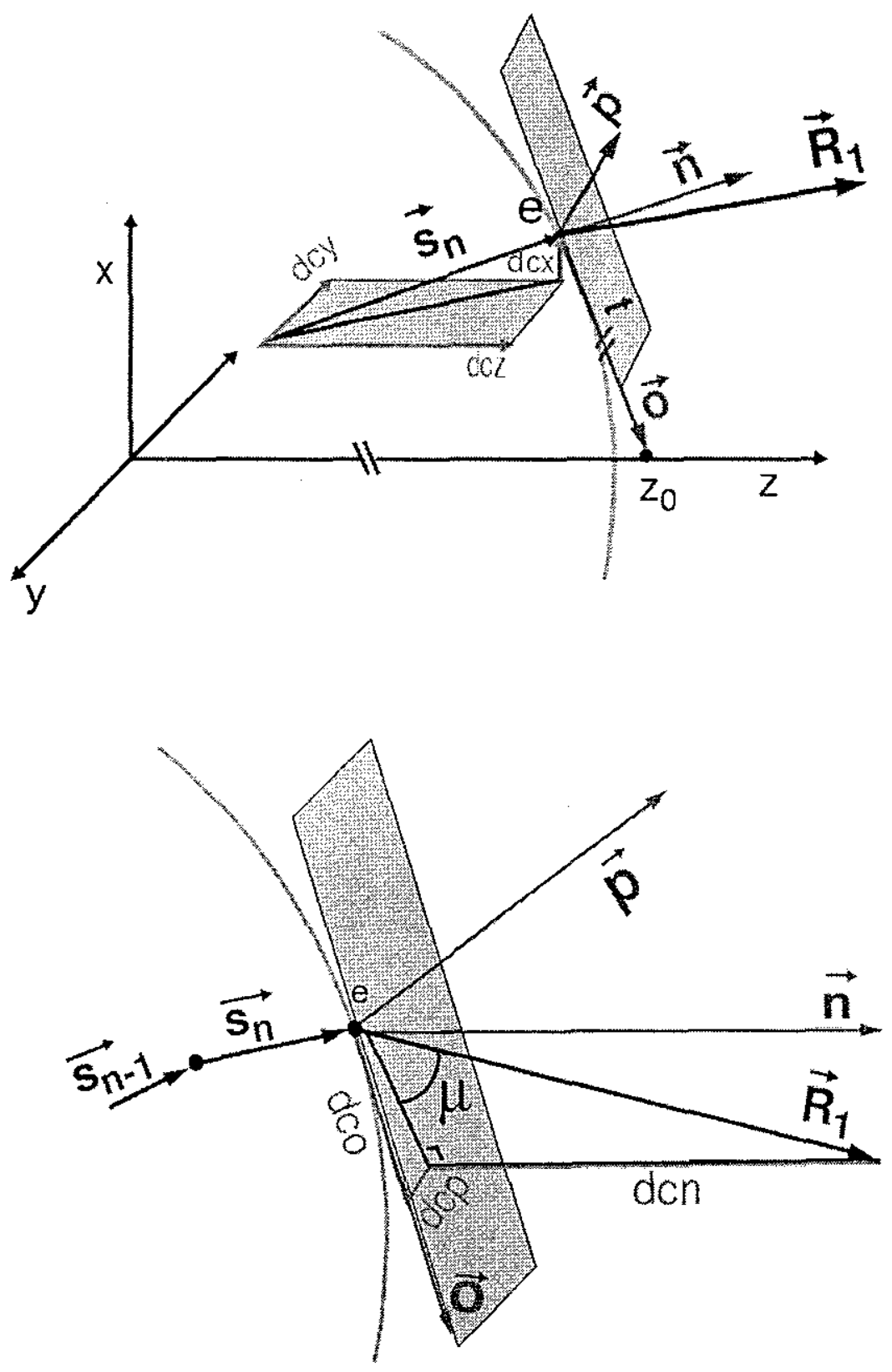

Figure 23: The local geometry for the electron's exit trajectory. The trajectory for the last step inside the kugel is represented by $\overrightarrow{s_{n}}$ and the trajectory of the exiting electron is shown by $\vec{R}_{1}$. 


$$
\begin{aligned}
& n_{1}=x_{k u g} / R_{k u g} \\
& n_{2}=y_{k u g} / R_{k u g} \\
& n_{3}=z_{k u g} / R_{k u g}
\end{aligned}
$$

where $\left(x_{k u g}, y_{k u g}, z_{k u g}\right)=e$ and $R_{k u g}$ is the kugel radius. The components of $\vec{o}$ are

$$
\begin{aligned}
& o_{1}=-x_{k u g} / t \\
& o_{2}=-y_{k u g} / t \\
& o_{3}=\left(z_{0}-z_{k u g}\right) / t,
\end{aligned}
$$

and the components of $\vec{p}$ are given by equation 3.5 :

$$
\begin{aligned}
& p_{1}=n_{2} \cdot o_{3}-n_{3} \cdot o_{2} \\
& p_{2}=n_{3} \cdot o_{1}-n_{1} \cdot o_{3} \\
& p_{3}=n_{1} \cdot o_{2}-n_{2} \cdot o_{1} .
\end{aligned}
$$

The direction cosines of the $\vec{R}_{1}$ trajectory relative to the new axes can be found by taking the projection of dcx, dcy, and dcz onto the new axes:

$$
\begin{aligned}
d c n & =d c x \cdot n_{1}+d c y \cdot n_{2}+d c z \cdot n_{3} \\
d c o & =d c x \cdot o_{1}+d c y \cdot o_{2}+d c z \cdot o_{3} \\
d c p & =d c x \cdot p_{1}+d c y \cdot p_{2}+d c z \cdot p_{3} .
\end{aligned}
$$




\section{Storing Trajectory Components}

As seen above, the exit trajectory is specified by three correlated direction cosines within the CREEP code, of which only two are completely independent since $d c x^{2}+d c y^{2}+d c z^{2}=1$.

The correct way to store trajectory components is to use a method that fully retains this correlation. To do this, one would store the probability of $d c n, P[d c n]$, and $P[d c o ; d c n]$, the probability of $d c o$ as a function of $d c n$. This would require a separate $d c o$ PDF for each element of $P[d c n]$, causing an increase of storage by the square of the number of bins in $P[d c n]$. It would also require longer running time of the local code to get adequate statistics.

One might be tempted to abandon correlation completely and store $P[d c n]$ and $P[d c o]$ in separate distributions. This has the untractable problem that it would be possible to sample two direction cosines that would sum to a quantity greater than one, which is a non-physical solution. Even if the values were normalized, the shape of the original distribution would be perturbed.

A better solution can be seen by revisiting the basic spherical coordinate scheme, shown in Figure 24. A direction can be specified by extending a vector from the center to a point on the unit sphere. This point is defined by two quantities: the azimuthal angle and the polar angle. It is the sines of these angles which are stored in the MRMC library.

In the defined kugel geometry, this corresponds to storing one of the direction cosines directly, $d c p=\sin \phi$, and storing the projection of the elevation angle from the tangent plane, $\mu$, where

$$
\sin \theta=\cos \mu=\frac{d c o}{\sqrt{d c o^{2}+d c n^{2}}}=\frac{d c o}{\sqrt{1-d c p^{2}}} .
$$

In effect, this is storing the other direction cosine with its dependency on $d c p$ 


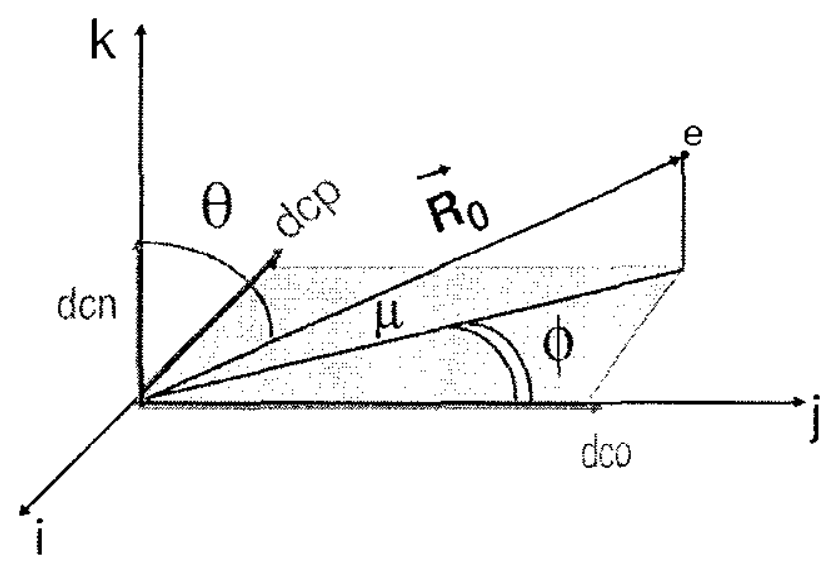

Figure 24: The spherical coordinate system uses two angles to define a point, or trajectory on the unit sphere.

factored out. In the global sampling code, the dependency can be reintroduced after a particular $d c p$ is selected, as will be shown in Chapter 4 . This prevents unphysical values.

Both distributions for the cosines of these angles are tallied from -1 to 1 . Although the $d c p$ and $\cos \mu$ are sampled from independent distributions and not correlated to each other, this difficulty is mitigated somewhat by the band structure.

\subsubsection{Knock-on Exit Energy}

The knock-on exit energy is tallied in the same manner as the primary; it is also normalized to the incident energy of the primary. However, the bins span only half the range required in the primary case, from 0 to 0.5 , since the knock-on by definition can have no more than half of the primary's energy. 


\subsubsection{Knock-on Position Cosine}

The knock-on position cosines are tallied in exactly the same way as in the primary case, with the extrema for $z_{k u g} / R_{k u g}$ determined by the extrema for the respective bands, as in the primary case. The position cosines for the knock-on distributions are spread out compared to that of their primary electrons due to the electrons' tendency to scatter through larger angles at lower energies.

\subsubsection{Knock-on Exit Trajectory}

The trajectory for the escaping knock-on electrons is tallied in exactly the same manner as it is in the primary case.

\subsubsection{Photon Energy}

As was discussed in the previous chapter, although most bremsstrahlung photons created have low energy, it is possible for an electron to give up all of its energy to a photon. Clearly it is important to allow for this possibility due to the large impact it would have on the overall distribution; thus the bremsstrahlung energy tallies run from 0 (none of the electron's energy) to 1 (all of the electron's energy).

\subsubsection{Photon Position Cosine}

The position of the photon at the point of the bremsstrahlung interaction is recorded as $z_{k u g} / R_{k u g}$. There is no band structure for photons. Any ambiguity about which band the photon would have escaped from if it could have been tracked to the surface is avoided since all positions are tallied in the same distribution. 


\subsubsection{Photon Trajectory}

The trajectory for the bremsstrahlung photon is computed and tallied in the same manner as it is for electrons, with the exception that it must be projected to the surface from its birth position.

\subsection{Other Tallies}

\subsubsection{Average Energy Expended}

The average energy expended in a kugel per history on a band-by-band basis can be used for expected energy loss calculations in the global calculation. The energy expended in the kugel is computed when the electron escapes from a particular band, by taking the difference between the incident energy and the exit energy. Thus any energy that has escaped with secondary particles is considered lost and is reflected in this quantity, even though the secondaries may be sampled and tracked on their own at a later step in the global code. More on this topic will be discussed in Chapter 5.

\subsubsection{Band Probabilities and Boundaries}

The number of primary (and, separately secondary) electrons leaving each band is counted and normalized by the total number of incident (or secondary) electrons in the problem to obtain the probability of escaping from each band. These probabilities, are stored in file along with the $z / R$ value for the boundary of each bin. Examining the values shown in Table 5 - Table 8 gives a sense of the peaked nature of the distributions for each particle type, for several incident energies and kugel sizes. 
Table 5: The probabilities of a primary electron escaping from each surface band for calcium $\left(\rho=1.6 \mathrm{~g} / \mathrm{cm}^{3}\right)$ with $\mathrm{R}_{k u g}=0.05 \mathrm{~cm}$, for four incident energies.

\begin{tabular}{c|c||c|c|c|c} 
Band & $\mathrm{z} / \mathrm{R}$ & $0.25 \mathrm{MeV}$ & $1 \mathrm{MeV}$ & $5 \mathrm{MeV}$ & $8 \mathrm{MeV}$ \\
\hline \hline 1 & 0.98 & $5.0 \mathrm{e}-6$ & 0.57 & 0.90 & 0.98 \\
\hline 2 & 0.80 & $4.2 \mathrm{e}-5$ & 0.25 & 0.09 & $1.6 \mathrm{e}-2$ \\
\hline 3 & 0.40 & $3.7 \mathrm{e}-5$ & 0.11 & $3.9 \mathrm{e}-3$ & $8.1 \mathrm{e}-4$ \\
\hline 4 & -1.0 & $7.7 \mathrm{e}-6$ & 0.06 & $1.0 \mathrm{e}-3$ & $2.2 \mathrm{e}-4$ \\
\hline
\end{tabular}

Table 6: The probabilities of a secondary "knock-on" electron escaping from each surface band for calcium $\left(\rho=1.6 \mathrm{~g} / \mathrm{cm}^{3}\right)$ with $\mathrm{R}_{k u g}=0.05 \mathrm{~cm}$, for four incident energies.

\begin{tabular}{c|c||c|c|c|c} 
Band & $\mathrm{z} / \mathrm{R}$ & $0.25 \mathrm{MeV}$ & $1 \mathrm{MeV}$ & $5 \mathrm{MeV}$ & $8 \mathrm{MeV}$ \\
\hline \hline 1 & 0.98 & 0 & 0.52 & 0.41 & 0.43 \\
\hline 2 & 0.80 & 0 & 0.25 & 0.42 & 0.39 \\
\hline 3 & 0.40 & 0 & 0.13 & 0.13 & 0.13 \\
\hline 4 & -1.0 & 0 & 0.10 & $4.1 \mathrm{e}-2$ & $4.6 \mathrm{e}-2$ \\
\hline
\end{tabular}


Table 7: The probabilities of a secondary bremsstrahlung photon escaping from each surface band for calcium $\left(\rho=1.6 \mathrm{~g} / \mathrm{cm}^{3}\right)$ with $R_{k u g}=0.05 \mathrm{~cm}$, for four incident energies.

\begin{tabular}{c|c||c|c|c|c} 
Band & $\mathrm{z} / \mathrm{R}$ & $0.25 \mathrm{MeV}$ & $1 \mathrm{MeV}$ & $5 \mathrm{MeV}$ & $8 \mathrm{MeV}$ \\
\hline \hline 1 & 0.98 & 0.02 & 0.04 & 0.83 & 0.89 \\
\hline 2 & 0.80 & 0.17 & 0.13 & $9.5 \mathrm{e}-2$ & $3.8 \mathrm{e}-2$ \\
\hline 3 & 0.40 & 0.30 & 0.28 & $3.0 \mathrm{e}-2$ & $2.7 \mathrm{e}-2$ \\
\hline 4 & -1.0 & 0.51 & 0.55 & $4.6 \mathrm{e}-2$ & $4.7 \mathrm{e}-2$ \\
\hline
\end{tabular}

Table 8: Band probabilities for several kugels of varying size for carbon ( $\rho=$ $2.0 \mathrm{~g} / \mathrm{cm}^{3}$ ) at an incident energy of $6.5 \mathrm{MeV}$.

\begin{tabular}{c|c||c|c|c} 
Band & z/R & $0.025 \mathrm{~cm}$ & $0.05 \mathrm{~cm}$ & $0.1 \mathrm{~cm}$ \\
\hline \hline 1 & 0.98 & .995 & 0.99 & 0.97 \\
\hline 2 & 0.80 & $4.0 \mathrm{e}-3$ & $8.8 \mathrm{e}-3$ & $2.3 \mathrm{e}-2$ \\
\hline 3 & 0.40 & $2.6 \mathrm{e}-4$ & $4.9 \mathrm{e}-4$ & $1.1 \mathrm{e}-3$ \\
\hline 4 & -1.0 & $8.6 \mathrm{e}-4$ & $1.3 \mathrm{e}-4$ & $2.5 \mathrm{e}-4$ \\
\hline
\end{tabular}




\subsubsection{Average Number of Knock-ons Escaping}

The number of knock-on electrons that escape the kugel for each incident history is counted. ${ }^{2}$ When the primary eventually leaves the kugel, the escape energy is tallied in an energy bin as described in Section 3.3.2 above. The bin number that is used in this energy tally is saved, and the same bin is incremented in an array which stores a count of knock-ons as a function of exit energy and band. When the output routines are called, the number of knock-ons escaping in each of those bins is divided by the number hits in the corresponding exit energy bin, resulting in the average number of knock-ons that escape along with the primary of a particular energy. This quantity is also relevant if the primary is absorbed (considered a "band 0" event in the code) because it is still possible that a secondary may have escaped even though the primary did not.

\subsubsection{Average Number of Photons Escaping}

The average number of bremsstrahlung photons that escape as a function of primary exit energy (which may be 0 ) is computed as a function of band in a manner analogous to that described for knock-ons above. Since CREEP does not transport the photons, they are all considered to escape the kugel.

\subsection{Energies, Sizes, and Materials for the Li- brary}

The storage required for the MRMC library is directly related to the number of kugels required to adequately reproduce the PDFs for a step of given size, energy

\footnotetext{
${ }^{2}$ Recall that the knock-ons are given priority in tracking as soon as they are created. The phase space of the primary is stored on a stack until the knock-on is escaped or absorbed, then its transport is resumed.
} 
and material type. There is no interpolation between kugels for size or material type; those choices must be made judiciously at library generation time. For the current energy, it is essential to be able to interpolate between a small number of library energies, called the "energy ladder".

\subsubsection{Energy Ladder}

The average energy loss and angular deflection increases exponentially with decreasing energy. Condensed history (class I) typically uses $8 \%$ energy loss between tabulated distributions, but Ballinger was able to use $25 \%$ energy loss between tabulated distributions by using logarithmic statistical interpolation between discrete cnergies [3]. Statistical interpolation is further explained in Section 4.4.

Logarithmic energy spacing of kugel library entries helps to control the amount of variation between PDF distributions. This increases the number of kugel data sets required at low energies, which are not of significant interest to many problems, including external beam radiotherapy. Therefore, a spacing corresponding to approximately $25 \%$ energy loss was used from the initial energy down to 0.25 $\mathrm{MeV}$, where most electrons are absorbed (within the $0.05 \mathrm{~cm}$ radius kugel). The energy ladder should be a function of material type and kugel size, however, for simplicity in this work a constant energy ladder was used.

\subsubsection{Sizes}

For the present work, three kugel sizes were generated, having radii of $0.025 \mathrm{~cm}$, $0.05 \mathrm{~cm}$, and $0.1 \mathrm{~cm}$. It was desirable to have three different sizes so that smaller steps could be taken near boundaries and larger steps through homogeneous regions of the phantom. However, all of these sizes are relatively small compared to the step sizes necessary to achieve large efficiency savings in a radiotherapy treatment planning simulation. For such an application, the transport zones (corresponding 
to a CT voxel) are on the order of $1 \mathrm{~mm}$ and ideally the MRMC method would take step sizes that would range from a tenth of a zone to several zones. However, the time to generate kugel data sets for kugel radii of $0.5 \mathrm{~cm}$ or larger is quite significant. For this reason, small kugels were chosen for this "proof-of-concept" work. As will be shown in subsequent chapters however, the transport mesh size was also scaled down, so that the method could still be tested for its ability to cross several zones at once.

\subsubsection{Materials}

One reason the MRMC method (or any Local-to-Global method) may be viable for radiation treatment planning is that there is a finite set of materials that are needed. A general-purpose code like MCNP or EGS4 may require a library that is prohibitively large. Clearly if there were hundreds of kugel data sets that needed to be loaded in for each global calculation, the size of the database to be accessed by the code would be unwieldy, not to mention the resources that would be required to generate it initially. The materials in the MRMC library will ultimately be chosen either for biological relevance, machine relevance or experimental benchmarking. For the present work only calcium and carbon kugel data sets are in the library. These were chosen because they are low Z, non-gaseous elements of clinical importance. Compounds, like water, and mixtures, such as air, will be important additions. Although a version of CREEP was built to generate these materials, it was never benchmarked thoroughly enough for inclusion here. 
Table 9: The size in bytes of the data files necessary for 1 kugel in uncompressed ASCII format.

\begin{tabular}{|c|c|}
\hline Filename & size $(\mathrm{B})$ \\
\hline \hline phsppdf.all & 147,600 \\
\hline secnum.all & 14,436 \\
\hline bandprbs.out & 300 \\
\hline TOTAL & 162,336 \\
\hline
\end{tabular}

\subsection{The Library Format}

All PDFs are written in the Alias format [22] for efficient sampling in the global code. This format is essentially a rejection technique which is $100 \%$ efficient because the PDF is rearranged to exactly fit the sampling space, as is adeptly explained by Du [21]. The PDFs may also be written to a file in a standard format, $[x, P(x)]$, for viewing. The distributions are not interpolated continuously in $x$ because any knowledge of the original $x$-value is lost in the histogramming process. Thus all the possible values within a bin are treated as equi-probable.

\subsection{Storage Requirements for the Library}

There are three essential files for each kugel in the MRMC library. The file "phsppdf.all" contains all of the phase space information for 3 particle types, each having 4 PDF types, each of which contain 100 bins of data in the Alias format. The file "secnum.all" contains the average number of secondaries emerging from the kugel as a function of 100 exit-energy bins, for both knock-ons and photons. The file "bandprbs.out" contains the cumulative probability of emerging from each surface band for each of the 3 particle types. The size of these files is listed in Table 9 . 
The real issue is the amount of storage required for a global calculation, which depends on the number of incident energies, sizes, and materials desired for a given problem. All of the results presented in Chapter 5 were generated using no more than 10 incident energies. For clinical calculations at energies higher than those presented in Chapter 5, a few high-energy kugels would need to be added to the library, but it is likely that the problem could be run without the same resolution at low energies (below $1 \mathrm{MeV}$ ). Assuming 10 incident energies are adequate, the total storage becomes 1.6 MB. Currently, the MRMC library contains data for 3 different size kugels, which brings the total to $4.86 \mathrm{MB}$ of data for a single material type.

Many clinical phantoms have only a few material types. However, a typical CT scan may have on the order of 10 different material types, requiring approximately $50 \mathrm{MB}$ for a CT-type calculation. A total database might have on the order of 100 materials to choose from, of which only the ones necessary for a given run would be loaded into the program at once. An estimate for a total, uncompressed kugel data library is therefore on the order of $500 \mathrm{MB}$, a large but manageable size.

\subsection{Library Generation and Navigation}

There are several scripts that are used with CREEP to generate kugel data sets. All of them rely on the directory structure of the library that was shown in Figure 21. In addition to the features shown, there is a file called "dirinfo" at each level which lists the number of subdirectories, and subsequently, the names of each subdirectory. The dirinfo files can either be generated automatically through a system command, or they can be edited by hand. Editing this file by hand at the "energy" level of the directory structure, for example, allows the user to define a particular subset of the available kugel energies to be read in for a specific run. The MRMC data preparation routines will only load data sets from subdirectories 
that are listed in the "dirinfo" files.

Because of the intense computational resources required to generate a kugel data set, several techniques have been implemented to minimize run time. The first two were used together to compile the existing kugel data sets for calcium and carbon for kugel sizes having a radius of $0.025 \mathrm{~cm}, 0.05 \mathrm{~cm}$ and $0.1 \mathrm{~cm}$. The bootstrap method described in Section 3.8.3 has not been fully tested, but is probably necessary to generate the larger kugel sizes which would be necessary for real CT volumes.

\subsubsection{Serial Kugel Generation}

By dispatching an entire suite of runs in series, no CPU time between runs is wasted and the user can easily generate an entire energy ladder in increasing order of kugels for a given material. It is convenient for debugging purposes to generate the kugels in increasing energy, so that low-energy problems can be tested while the high-energy kugels are running.

\subsubsection{Nested Kugel Generation}

In the concentrically nested configuration, several different kugel sizes of the same incident energy and material are run concurrently. Since the kugels begin with transport in the center, the results from the inner spheres can be saved and used as partial histories for the larger spheres. This is illustrated in Figure 25.

The electrons are incident at point $P_{0}$ and are each transported up to the innermost radius, $R_{A}$, first. As they exit, their phase space is both tallied for the kugel data set at $R_{A}$, and held in a "hub" bank for later transport through the next radius $R_{B}$. After all the incident electrons have been transported to the innermost radius, the transport to the next radius continues by using all the electrons in the 


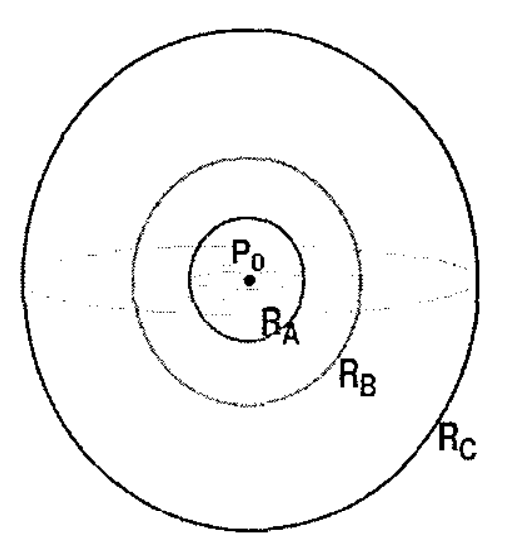

Figure 25: Three kugels in a concentrically nested configuration. The electrons for all three kugels are incident at point $P_{0}$.

"hub" bank as source particles. The present radius, $R_{B}$, is considered the innermost radius, and transport continues to $R_{C}$, and so on. This method guarantees that there is a smooth transition between radii, and backscattered electrons are neither lost nor double-tallied.

\subsubsection{Bootstrapped Kugel Generation}

The concept of generating large kugels by "bootstrapping" up from smaller kugels is probably necessary to achieve reasonable run times for clinically-sized kugels. This method is a superposition of the local and global codes. It uses the local geometry and tracking, but the global sampling routines to determine the state of the electron after a step that is the size of a small kugel which has complete data in the library already. This method is shown in Figure 26.

A drawback of this method is that any errors in sampling from the small kugel's data set will be compounded in the large kugel's data set. These errors will be compounded further when the large kugel is used in the global transport stage, which is described in the next chapter. Thus a detailed error analysis of this 


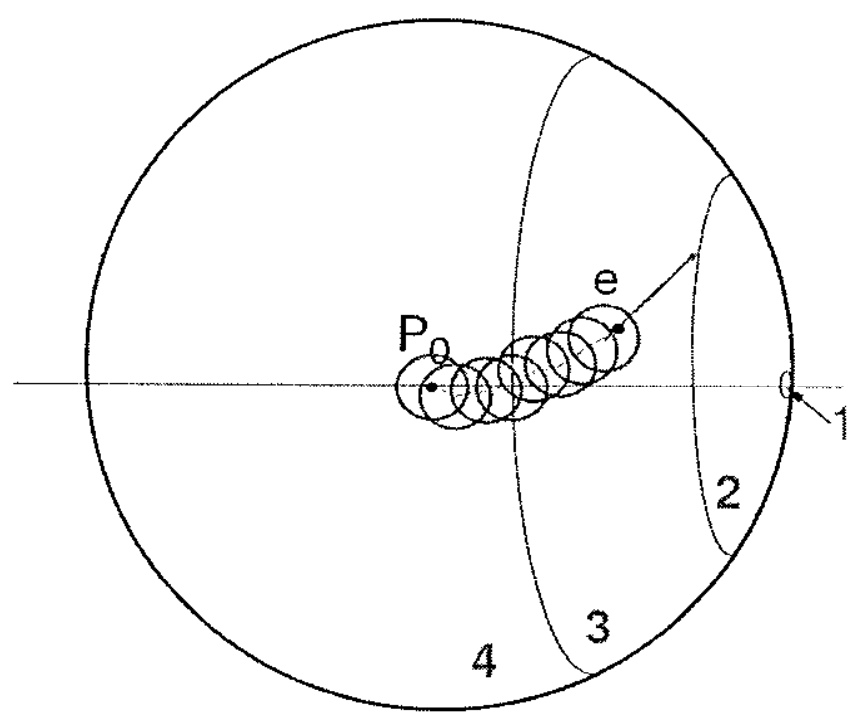

Figure 26: The "bootstrap" method generates a large kugel by using steps that are the size of a small kugel. The electron's phase space at the end of a step is sampled with the same routines as are used in the global MRMC stepping code, which is the subject of the next chapter. 
method is necessary. For this work, most of the research was done on small kugels, and the data sets generated with the bootstrap method were not thoroughly tested. It is a promising area for future studies. 


\section{Chapter 4}

\section{Macro Response Transport}

\subsection{Introduction}

The fundamental idea behind Macro Response transport is to take large spatial steps through homogeneous areas of the medium, often spanning multiple transport zones. A stylized two-dimensional representation of this idea is shown in Figure 27. Chapter 2 explained how Analog Monte Carlo works for electrons to provide accurate phase data (energy, position and trajectory) and Chapter 3 explained how this data can be used to compile sets of distributions for homogeneous spheres. The present chapter will discuss how these data sets can be used for efficient transport through a larger "global" medium.

\subsection{Logic Flow}

The logic flow for the global transport process is diagrammed in Figure 28.

Prior to transport, the appropriate data sets from the kugel library are read into arrays. This includes data for each material found in the medium and each energy needed. At least one data set tabulated with an incident energy higher than the incident energy in the global transport is required, due to the statistical interpolation scheme for the electron's current energy, described in Section 4.4.

The electron is initiated in a user-defined source routine which sets its initial encrgy, $E_{0}$, trajectory and position. MRMC tracking begins by checking if the 


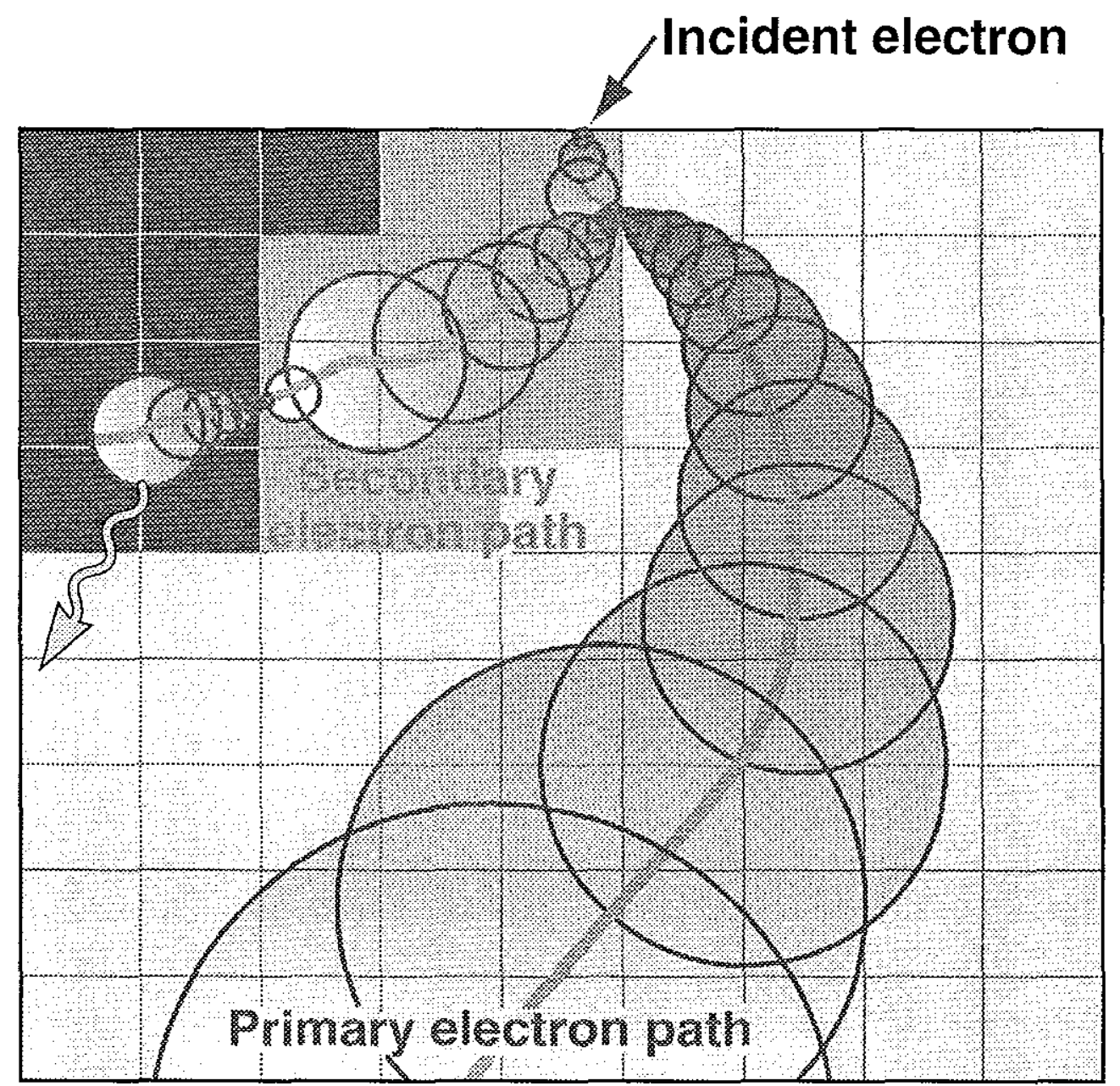

Figure 27: Transport in the Macro Response method is done by taking large steps through homogeneous areas of the mesh. In this picture, the electron is incident obliquely on the "top" of the transport mesh and the primary escapes the mesh at the bottom, while a secondary electron is absorbed after emission of a bremsstrahlung photon. The exit position from one kugel serves as the center of the next kugel. Secondary electrons are tracked in the same manner as primary electrons. Secondary photons are passed off to a parent code for tracking. The transport through each kugel is described in detail in Section 4.7.2. 


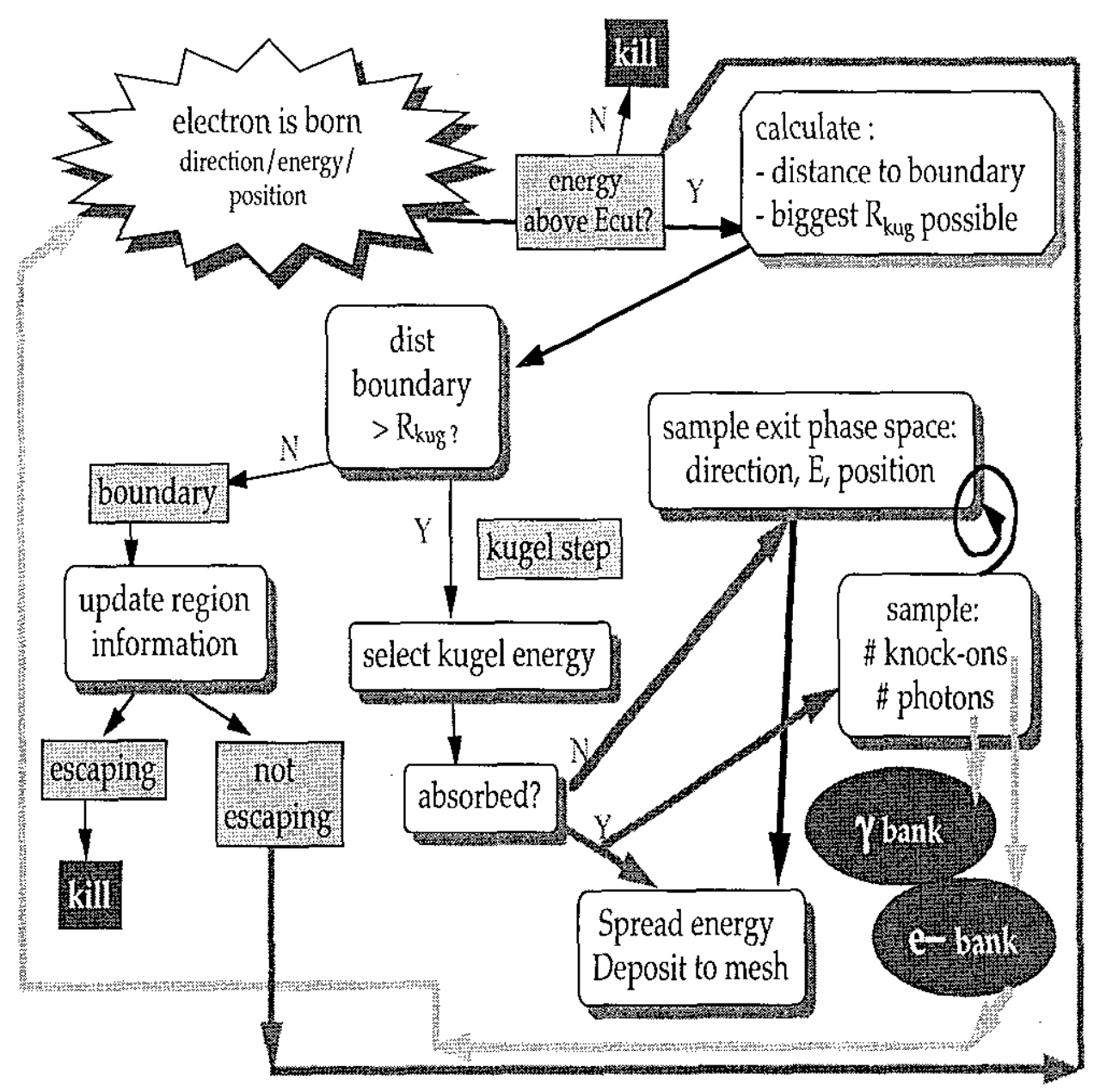

Figure 28: The logic flow for Macro Response Monte Carlo transport. 
electron has enough room to take a step before it hits a boundary. If so, it determines how large a kugel it can use, and selects an appropriate energy for the data set from the choices in the library. An exit band on the kugel surface is selected (see Section 4.5). If it is zero, the electron was absorbed and its energy is sent immediately to the energy-spreading routine described in Section 4.7. Otherwise, the electron escaped and MRMC then samples the outgoing phase space the electron will have at the end of the step, which includes exit energy, exit position, number of knock-on electrons that escape the kugel, number of bremsstrahlung photons created in the kugel, and the direction cosines that describe the electron's trajectory as it leaves the kugel. Position, energy, and trajectory must also be sampled for each of the knock-on electrons and bremsstrahlung photons that are created. These quantities are written to a bank for later tracking; the knock-on electrons are picked up at the end of the primary's history, and the photons are passed off to the parent tracking code.

All the phase space distributions are stored in a special format for expedient sampling with the Alias sampling algorithm [22]. This method has been reviewed by Ballinger [3] and Du [21].

MRMC transport is completed when all the electrons in the primary bank have been tracked, at which time an output routine tabulates information about the run and a post-processing routine in the parent program converts the energy deposited in each zone to absorbed dose.

\subsection{Determination of Step Size}

The MRMC library contains data for kugels of a few different radii. A large kugel size is desirable to save time in the global calculation, but a small size may be necessary when the electron is approaching a boundary. Thus selecting the optimal kugel radius available for a given step depends on the distance to 
the nearest boundary along the direction of travel, $d_{b}$. If some boundaries are to be considered "significant" and others not (such as the boundary between two materials of nearly the same density) the distance-to-boundary routine needs to include that information in its calculation. Once we know the maximum distance that can be traversed along the current trajectory, the largest kugel radius that is less than $d_{b}$ is selected. Since $d_{b}$ is only checked in the direction of travel, the kugel may have some overlap with the new material. This case is discussed in Section 4.8 .

\subsection{Determining the Kugel Energy}

The MRMC library contains data from local calculations that were initiated with many different incident energies. It is necessary to select one of these energies to represent the current electron. The array of possible values is searched to determine the two closest candidates, $E_{h i}$ and $E_{l o}$. A linear-logarithmic statistical interpolation is done to randomly choose between them. The value of $E_{h i}$ is chosen if the random number $\chi$ is such that

$$
\chi>\frac{\log E_{h i}-\log E}{\log E_{h i}-\log E_{l o}}
$$

otherwise $E_{l o}$ is chosen.

This interpolation differs from conventional logarithmic interpolation, in which an intermediate value between the endpoints is selected. Since the discrete energy points in the energy ladder represent the only choices available, it is the frequency with which the bounding values are chosen that tunes the interpolation. The interpolation is "statistical" in the same sense that the Monte Carlo method as a whole is a statistical process, as was introduced in Section 1.5. Each selection of energy is a valid choice, but a single choice (probably) does not adequately 
represent the electron's real energy. Rather, it is through the process of making these choices many times that the correct overall energy distribution emerges.

After many histories, then, provided the data sets are not too sparse in energy, the resulting energy distribution is correct. If the kugel data sets are not sufficiently spaced in energy, the linear-log statistical interpolation is not valid and causes the resulting energy distribution to favor one of the remaining energies.

\subsection{Determining the Exit Band}

The cumulative transmission probability for each band (beginning with band 1, since it is chosen most often for most kugels) is read into an array for global transport. Once the size and energy of the kugel have been defined, the exit band is determined simply by comparing a random number, $\chi^{\prime}$ to the cumulative probabilities. The band chosen is the first band for which

$$
P_{i}>\chi^{\prime}
$$

where $P_{i}$ is the cumulative probability of choosing the $i^{\text {th }}$ band.

\subsection{Updating Phase Space After A Step}

Assuming that the electron has been transmitted through the kugel, i.e., band 1-4 was chosen, the parameters that describe the electron's state as it exits the kugel must now be sampled.

\subsubsection{Determination of Exit Position}

The position along the z-axis is tallied within each band as $z_{k u g} / R_{k u g}$, where the limits on $z$ are set by the extrema of the bands. Once the z-position has been 


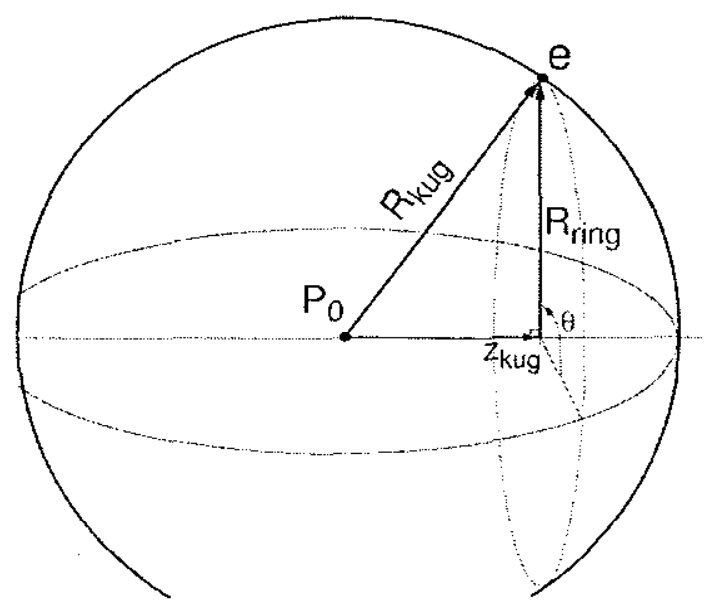

Figure 29: The geometry for the determination of the exit position. The random angle $\theta$ defines a point on the ring on the surface of the kugel at distance $z_{k u g}$ from the kugel's center.

determined, the exit position is constrained to the ring of radius $R_{\text {ring }}$ at the surface, about which it is evenly distributed. The $\mathrm{x}$ and $\mathrm{y}$ positions are functions of a random angle, $\theta$ between 0 and $2 \pi$.

$$
\begin{aligned}
& x=R_{\text {ring }} \sin \theta \\
& y=R_{\text {ring }} \cos \theta
\end{aligned}
$$

where

$$
R_{\text {ring }}=\sqrt{R_{\text {kug }}^{2}-z^{2}} .
$$

This geometry is illustrated in Figure 29. 


\subsubsection{Determination of Exit Trajectory}

Chapter 3 mentioned the importance of choosing the proper coordinate frame for accurate sampling of direction cosines. MRMC uses a reference frame defined by the surface normal, $\vec{n}$, at the sampled exit point, $e$, as the vector relative to which the trajectory is tallied; the axis along $\vec{o}$, which is defined as the vector which is normal to $\vec{n}$ and intersects the kugel's z-axis at point $z_{0}$, which is a distance $t$ from $e$; and the axis along $\vec{p}$, which defined by

$$
\vec{p}=\vec{n} \times \vec{o} .
$$

This geometry is illustrated in Figure 30 .

A few steps are necessary to extract the two direction cosines needed to define the new trajectory. First, the two cosines are sampled from their respective distributions: $d c p$; and $\cos \mu$, the cosine of the projection of the elevation angle from the tangent plane. The remaining two direction cosines can be defined by

$$
\begin{aligned}
& d c o=\cos \mu \cdot \sqrt{1-d c p^{2}} \\
& d c n=|d c o| \cdot \sqrt{\left(1 / \cos \mu^{2}\right)-1}
\end{aligned}
$$

Note that $d c n$ is always positive due to the electron emerging from the kugel.

Since these quantities are defined in terms of a frame of reference normal to the kugel surface, the axis of this frame must be defined in terms the present kugel axes. The components of $\vec{n}$ are given by

$$
\begin{aligned}
& n_{1}=x_{k u g} / R_{k u g} \\
& n_{2}=y_{k u g} / R_{k u g}
\end{aligned}
$$



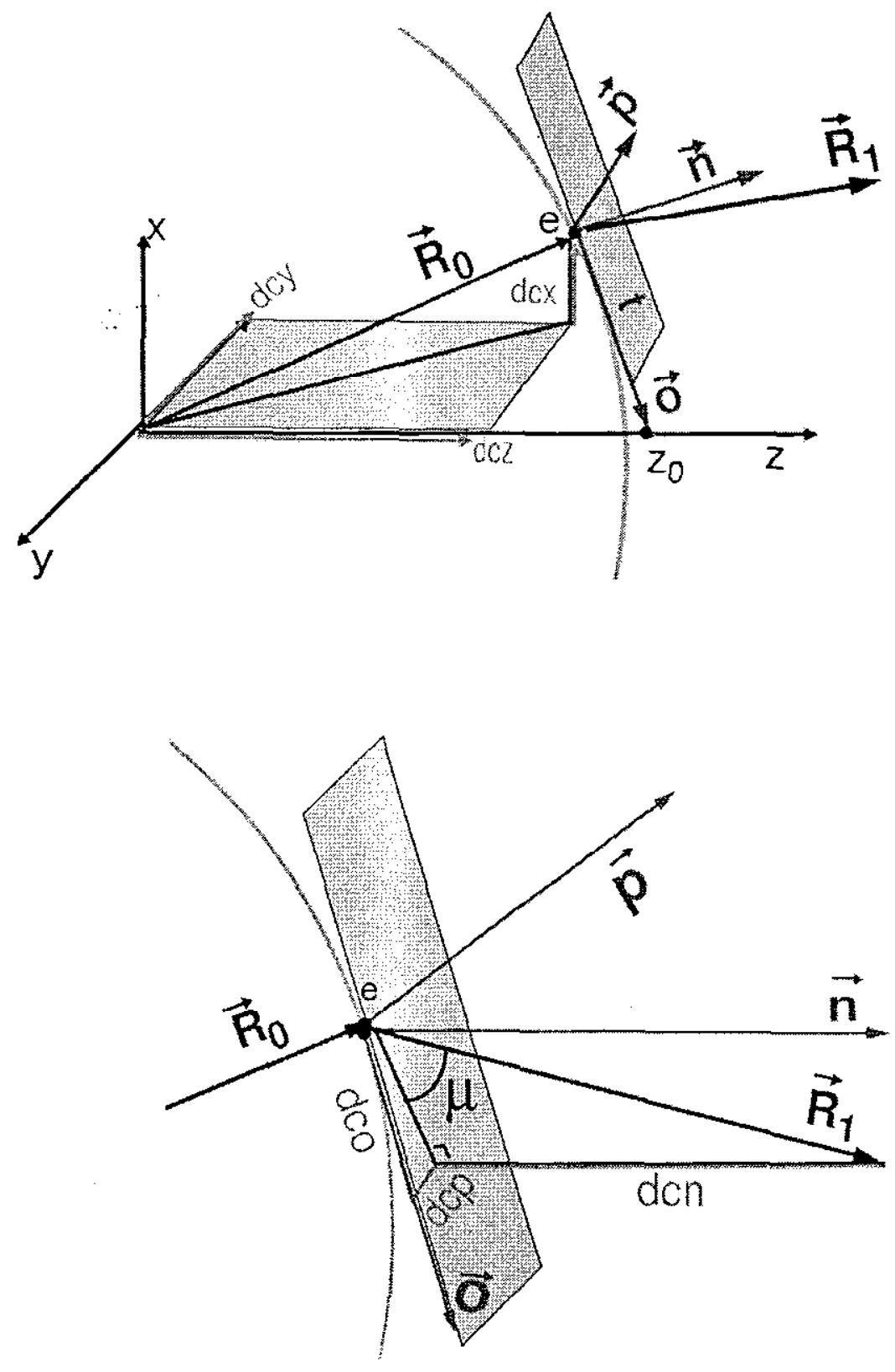

Figure 30: The global geometry for direction cosines. The trajectory through the current step is represented by $\vec{R}_{0}$ and the trajectory of the exiting electron is shown by $\overrightarrow{R_{1}}$. 


$$
n_{3}=z_{k u g} / R_{k u g}
$$

where $\left(x_{k u g}, y_{k u g}, z_{k u g}\right)=e$ and $R_{k u g}$ is the kugel radius. The components of $\vec{o}$ are

$$
\begin{aligned}
& o_{1}=-x_{k u g} / t \\
& o_{2}=-y_{k u g} / t \\
& o_{3}=\left(z_{0}-z_{k u g}\right) / t,
\end{aligned}
$$

and the components of $\vec{p}$ are given by equation 4.6 :

$$
\begin{aligned}
& p_{1}=n_{2} \cdot o_{3}-n_{3} \cdot o_{2} \\
& p_{2}=n_{3} \cdot o_{1}-n_{1} \cdot o_{3} \\
& p_{3}=n_{1} \cdot o_{2}-n_{2} \cdot o_{1} .
\end{aligned}
$$

This allows the sampled angles to be expressed in terms of the kugel frame, as

$$
\begin{aligned}
& d c x_{k u g}=\left(d c p \cdot p_{1}\right)+\left(d c o \cdot o_{1}\right)+\left(d c n \cdot n_{1}\right) \\
& d c y_{k u g}=\left(d c p \cdot p_{2}\right)+\left(d c o \cdot o_{2}\right)+\left(d c n \cdot n_{2}\right) \\
& d c z_{k u g}=\left(d c p \cdot p_{3}\right)+\left(d c o \cdot o_{3}\right)+\left(d c n \cdot n_{3}\right)
\end{aligned}
$$

Then the direction cosines must be transformed from the kugel frame to the global phantom frame in the same manner they were updated in the single scatter code, CREEP. 


\subsection{Energy Deposition to the Transport Mesh}

The importance of a good energy deposition algorithm, especially when the entire code is primarily benchmarked by depth dose curves, cannot be overestimated. In particular, when the kugels are allowed to cross several transport zones (which is the key to saving computation time), allocating energy amongst those zones in a realistic way is one of the most significant challenges facing this method of transport.

\subsubsection{Magnitude of Energy Deposition in Kugel}

Energy deposition is not a quantity that is tabulated directly in the kugel data set. Instead, it is deduced by sampling an exit energy for the primary electron, as well as the exit energies for any secondary particles that cscape. After these quantities are sampled, the "sampled" kugel deposition, $\epsilon_{\text {samp }}$, is given by

$$
\epsilon_{\text {samp }}=E_{\text {init }}-E_{\text {exit }}-\left(\sum_{i=1}^{N_{\text {pho }}} E_{p h o, i}+\sum_{j=1}^{N_{k o n}} E_{k o n, j}\right)
$$

where $E_{\text {init }}$ is the initial energy of the electron at the center of the kugel, $N_{\text {pho }}$ and $N_{k o n}$ are the number of escaping secondary photons and knock-ons, respectively, and $E_{p h o, i}$ and $E_{k o n, j}$ are the sampled exit energies of the $i^{\text {th }}$ photon and the $j^{\text {th }}$ knock-on electron. This equation may result in negative depositions, due to a lack of correlation between the exit energy of the primary electron and the total energy sampled for the secondaries. The negative depositions are statistically valid and necessary to achieve the proper energy distribution in the mesh.

It may be tempting to save some sampling time by simply using the average energy deposited per step in the chosen band. In fact this quantity is tallied and was used in the global transport code experimentally, but abandoned due to poor 
results. Using the average quantities eliminates the energy straggling "wings" of the distribution, as can be seen in Figure 31. These wings are necessary to reproduce features of electron behavior such as the tail of the depth dose curve, which is the result of many small depositions.

\subsubsection{Distribution of the Deposition Across Several Zones}

When the kugel is large enough to encompass many zones, there are several factors that affect the way the energy deposition should be shared between the zones. First a path must be assumed through the kugel that begins at the center and ends at the sampled exit position. Two potential paths, a simple radial path and a 3 segment path, were evaluated in this work and are illustrated in Figure 32. The extent to which this assumed path traverses through each of the transport zones is one factor that governs deposition in each of those zones. The stopping power is another factor, since it changes as the electron slows down en route to the edge of the kugel.

\section{Zone Weighting along the Path}

With the above in mind, it is clear that the energy-spreading algorithm must count the number of zones traversed and store the partial pathlengths through each. MRMC does a table lookup to obtain the stopping power ${ }^{1}$ at the beginning of each zone (or every group of zones) and calculates an "expected loss", $\langle\Delta \epsilon\rangle_{i}$ after crossing each $i_{\text {th }}$ zone, by multiplying the stopping power at the current energy times the partial pathlength. The energy in the subsequent zone is found by subtracting off $<\Delta E_{\text {loss }}>_{i-1}$.

When this process has been completed for all the $N$ zones, the expected energy loss for the kugel, $\langle\Delta \epsilon\rangle_{k u g}$ is known and used to normalize all the zonal losses.

\footnotetext{
${ }^{1}$ Stopping powers were generated from the technique outlined in ICRU 37 [47].
} 


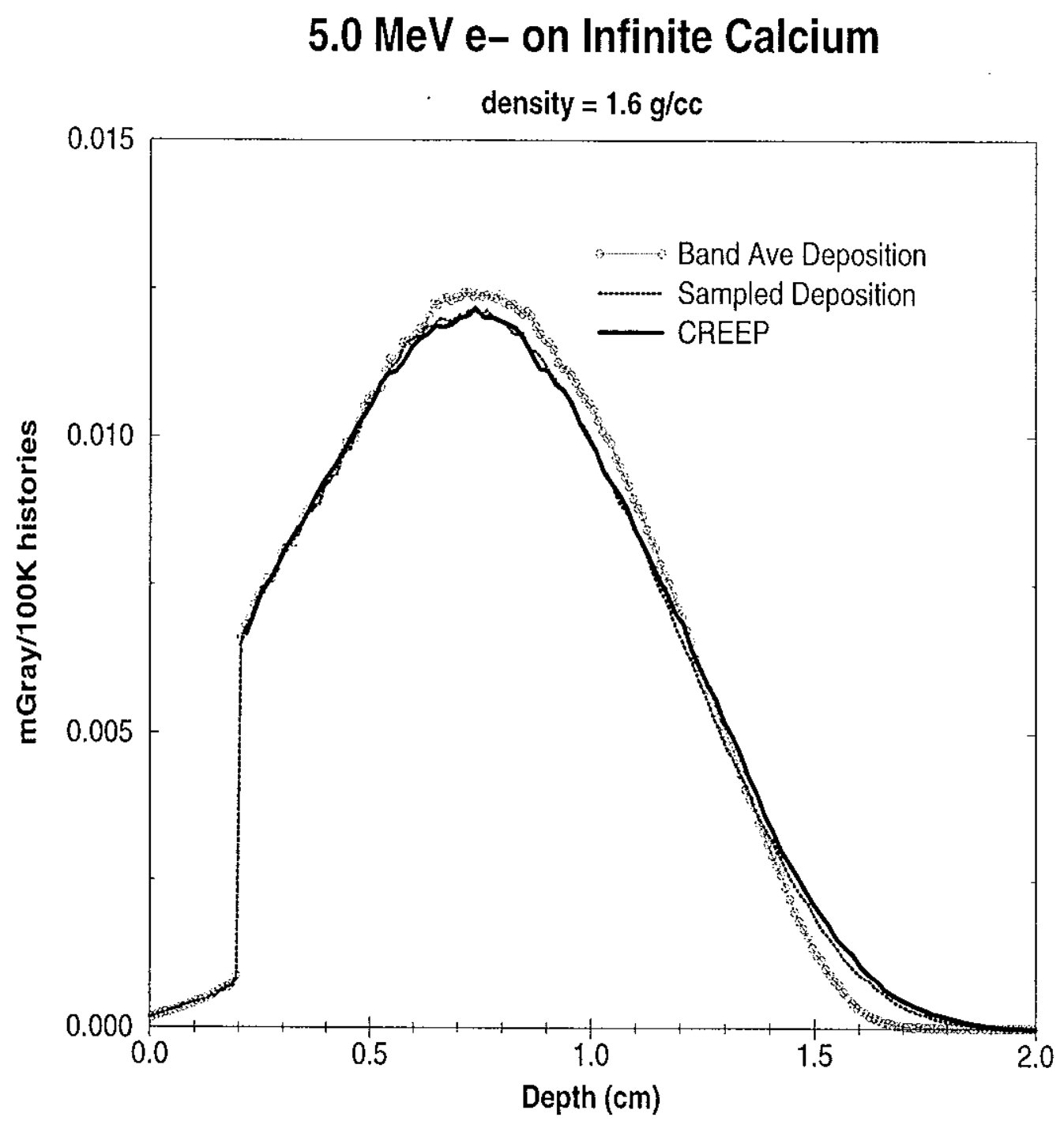

Figure 31: The difficulty with using the average energy deposition for the chosen band, as opposed to using the energy deposition given by equation 4.21 , which depends on the sampled outgoing energy at each step. The tail of the average deposition curve is cut short. The mean range of a $5 \mathrm{MeV}$ electron in calcium is $1.85 \mathrm{~cm}$, according to ICRU Report 37 [47], which is in good agreement with the "Sampled" and "CREEP" deposition curves. The phantom is an infinite calcium block with a source embedded at $0.2 \mathrm{~cm}$. 


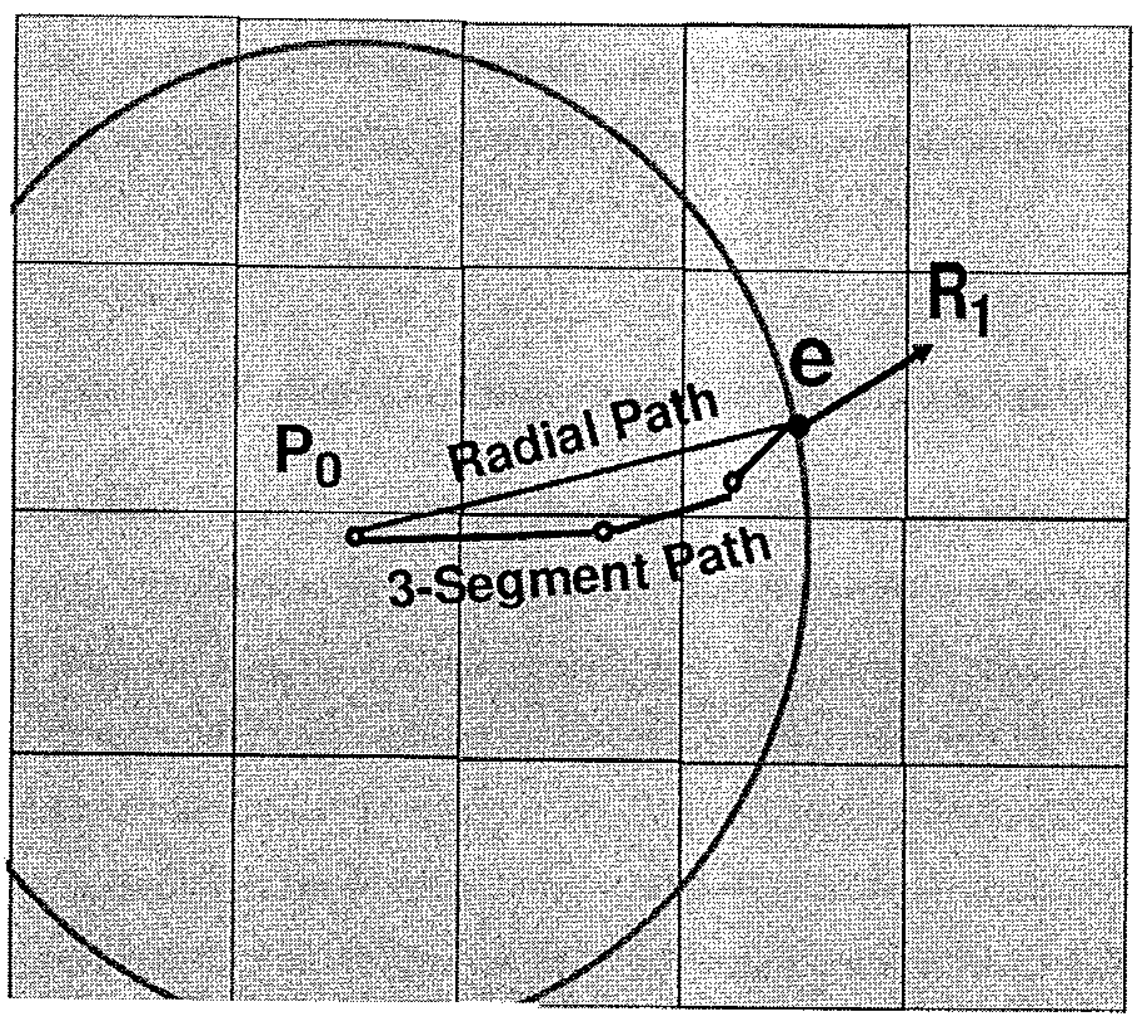

Figure 32: Two possible representations of the average electron path through the kugel for the purpose of energy deposition. The electron is incident at $P_{0}$, the center of the kugel, and escapes at the sampled point $e$ along the trajectory $R_{1}$. 
Thus the deposition in each of the $N$ zones that are determined to lie in the electron's path is given by

$$
\epsilon_{i}=\frac{S(E) \cdot p_{i}}{<\Delta \epsilon>_{k u g}} \cdot \epsilon_{\text {samp }}
$$

where $\epsilon_{\text {samp }}$ is the sampled energy loss for the whole kugel given by equation $4.21, p_{i}$ is the pathlength through the $i^{\text {th }}$ zone and $\mathrm{S}(\mathrm{E})$ is the restricted electron stopping power.

\section{Choosing a Path through the Kugel}

If the kugel size is on the order of a transport zone, a simple radial path extending directly from the center of the kugel to the sampled exit point may be adequate, as has been seen in prior work in this area [44]. However, in order to achieve increased time savings, MRMC deposits energy across multiple zones (typically 5-15). As can be seen in Figure 33, a more sophisticated algorithm is necessary in this case. The simple radial path overestimates the deposition in the first zones, and underestimates the deposition in the last zones, as is revealed by the periodic bumps in the portion of the depth dose distribution shown.

The reason for this can be found by examining the differential cross section for elastic scatter, shown in equation 2.5. Just as the electron stopping power changes as it loses energy, the scattering power also changes. The electrons begin their path traveling relatively straight, doing most of the scattering toward the edge of the kugel. The degree of this tendency depends on the atomic number of the material, its density and the incident energy. A route in the first few zones of the kugel which is along the z-axis will result in shorter partial paths through them, which causes a lower fraction of the deposition to be allocated to those zones. 


\subsection{MeV in Calcium}

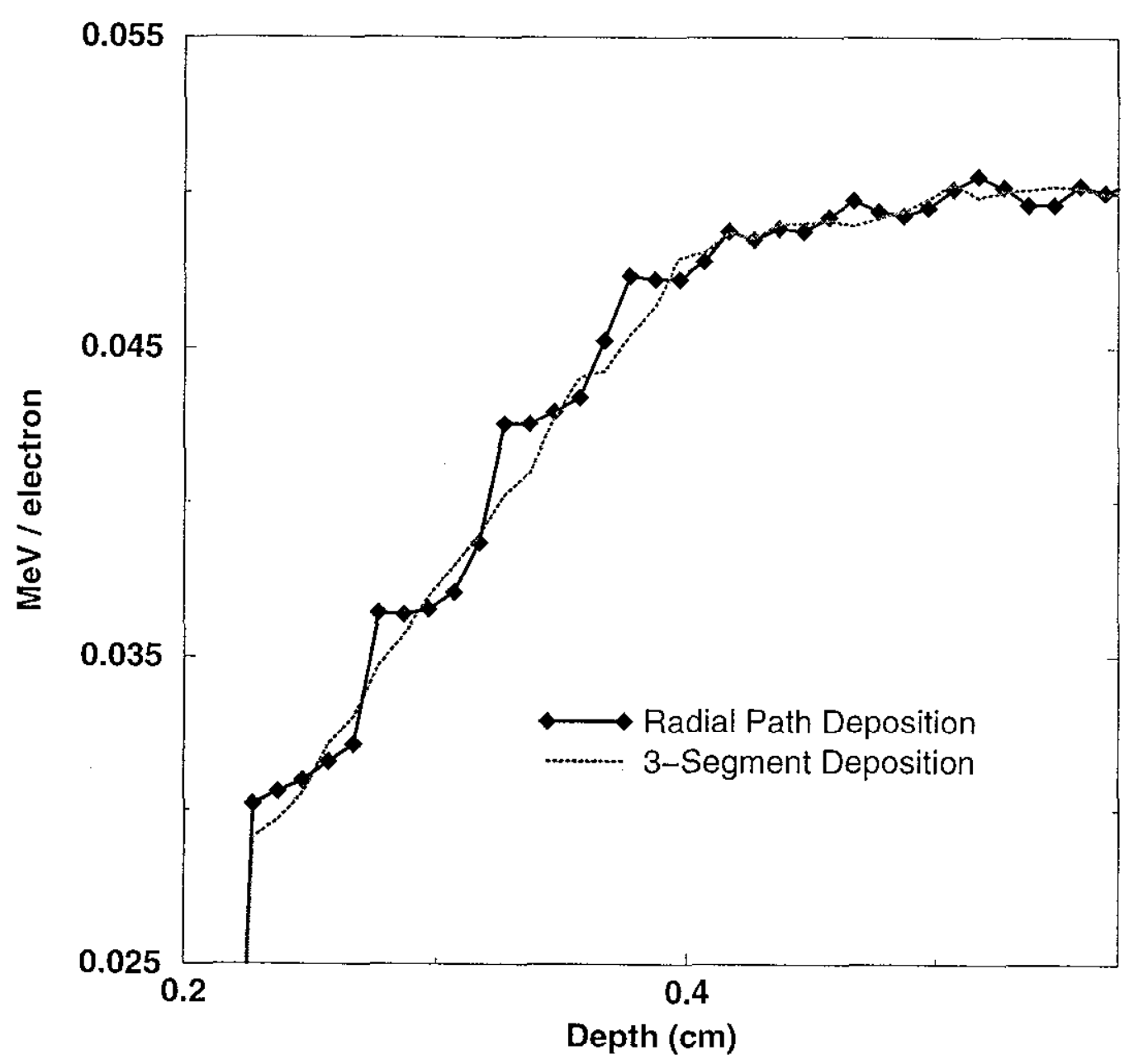

Figure 33: A comparison showing the step size artifacts associated with the simple radial path deposition when the kugel crosses several zones (here approximately 5 , since $R_{k u g}=0.05 \mathrm{~cm}$ and each transport zone is $0.01 \mathrm{~cm}$ deep). The periodicity of the radial path deposition results demonstrates the need for a more sophisticated energy deposition path through the kugel, "3-Segment Deposition" which is explained in Section 4.7.2. The source is embedded at $0.2 \mathrm{~cm}$ in the infinite calcium phantom. 
A first step towards a more physical path through the kugel is to allow the electron to travel some distance down its z-axis before preceding to the exit position. In fact, the path can be forced to meet the "boundary conditions" of this problem. The initial condition is that the orientation of the kugel was chosen so that the electron's trajectory would be aligned with its z-axis. The final condition is also known: it is the exit trajectory that is sampled for the next step. Thus the path must approach the exit position traveling along the exit trajectory. Two segments of the path are known, then, but the challenge remains to find an appropriate length for each of these segments, as well as representing the path in between the first and final segment.

\section{The Significance of Segment Lengths}

Choosing the lengths of the first and final segments was done phenomenologically for this work; this is an area where future research is needed. For the path in between the first and last segments, the simple approximation of a single straight path was used. Thus the path was broken into three segments having two articulation points, $P_{1}$ and $P_{2}$, as well the initial point, $P_{0}$, (the center of the kugel) and the exit point, $e$. This is illustrated by a two dimensional representation in Figure 34.

Using this scheme, the length of the middle segment, $l_{2}$, depends on the distance between $P_{1}$ and $P_{2}$. The first and last segments have a length given by

$$
\begin{aligned}
& l_{1}=(A+B \chi) \cdot R_{k u g} \\
& l_{3}=C \cdot R_{k u g},
\end{aligned}
$$

where $\chi$ is a random number, and $A, B$, and $C$ are coefficients that are chosen to minimize step size artifacts in the depth dose curve. For example, a calcium kugel 


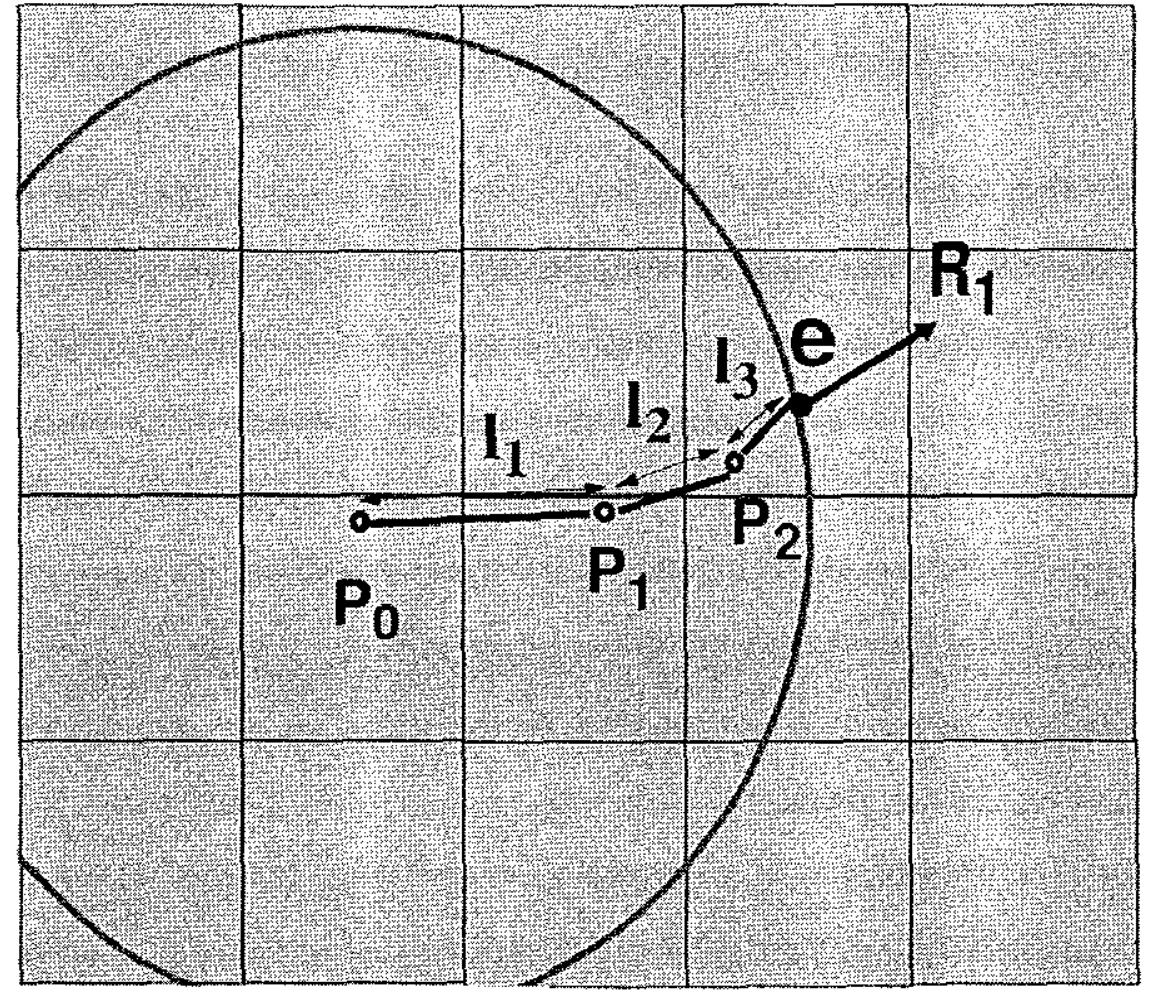

Figure 34: The three-segment path through the kugel. The electron is incident at $P_{0}$ and exits at the point $e$ along trajectory $\vec{R}_{1}$. 
of density $1.6 \mathrm{~g} / \mathrm{cc}$, radius $0.05 \mathrm{~cm}$, and an incident energy of $2.75 \mathrm{MeV}$ was found to have a smoothly varying curve using $A=0.1, B=0.5$, and $C=0.2$, while the same kugel with an incident energy of $5.0 \mathrm{MeV}$ was found to have good results with $A=0.3, B=0.6$, and $C=0.1$. Samples of the paths resulting from these values are shown in Figure 35 and Figure 36.

\subsection{Boundary crossing}

A boundary is defined as either as an interface between two different materials, or an interface between two densities of the same material whose difference exceeds a user-defined threshold. ${ }^{2}$ To calculate the correct energy deposition in the vicinity of a boundary, an electron is prevented from crossing the boundary as it travels on its path through a kugel. This is because the phase space distributions for its outgoing state would no longer be valid, even if the electron completes its route through the kugel in the same material in which it started.

Boundary crossing inside of a kugel is prevented in two ways. First, the distance to the nearest boundary intersecting the electron's trajectory, $d_{b}$, sets the limit for the step size. However, it is still possible that the projected pathlength through the kugel can hit a zone of new material as it deviates from the incident trajectory. As explained above, the energy deposition routine in this instance only deposits up to the new material and then aborts, beginning a new kugel on the other side of the boundary.

In the new material, the incident trajectory of the electron is the same as the trajectory it was following as it detected the boundary; the position is bumped incrementally by a distance $\Delta b$ into the new material along this trajectory. The new kugel has this point as its center, which implies that it is effectively "straddling"

\footnotetext{
${ }^{2}$ In the Peregrine implementation of MRMC, the edge of the phantom is detected when it hits a false border of material type " 0 ".
} 

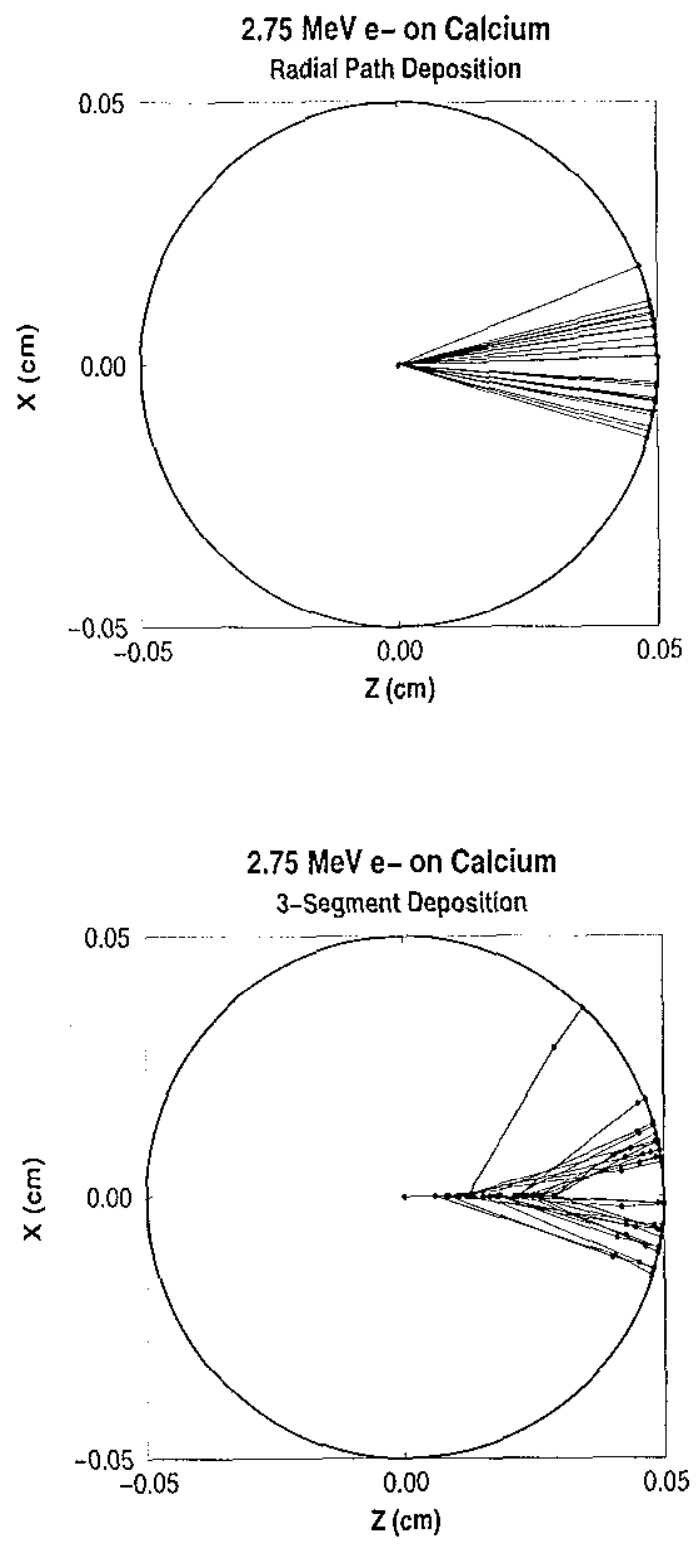

Figure 35: A comparison of paths through the kugel generated from the two algorithms tested. UPPER : $2.75 \mathrm{MeV}$ incidence; simple radial deposition $\left(l_{1}=0\right.$; $\left.l_{3}=0\right)$. LOWER : $2.75 \mathrm{MeV}$ incidence; $\left(l_{1}=(0.1+0.5 \chi) R_{k u g} ; l_{3}=0.2 R_{k u g}\right)$. 

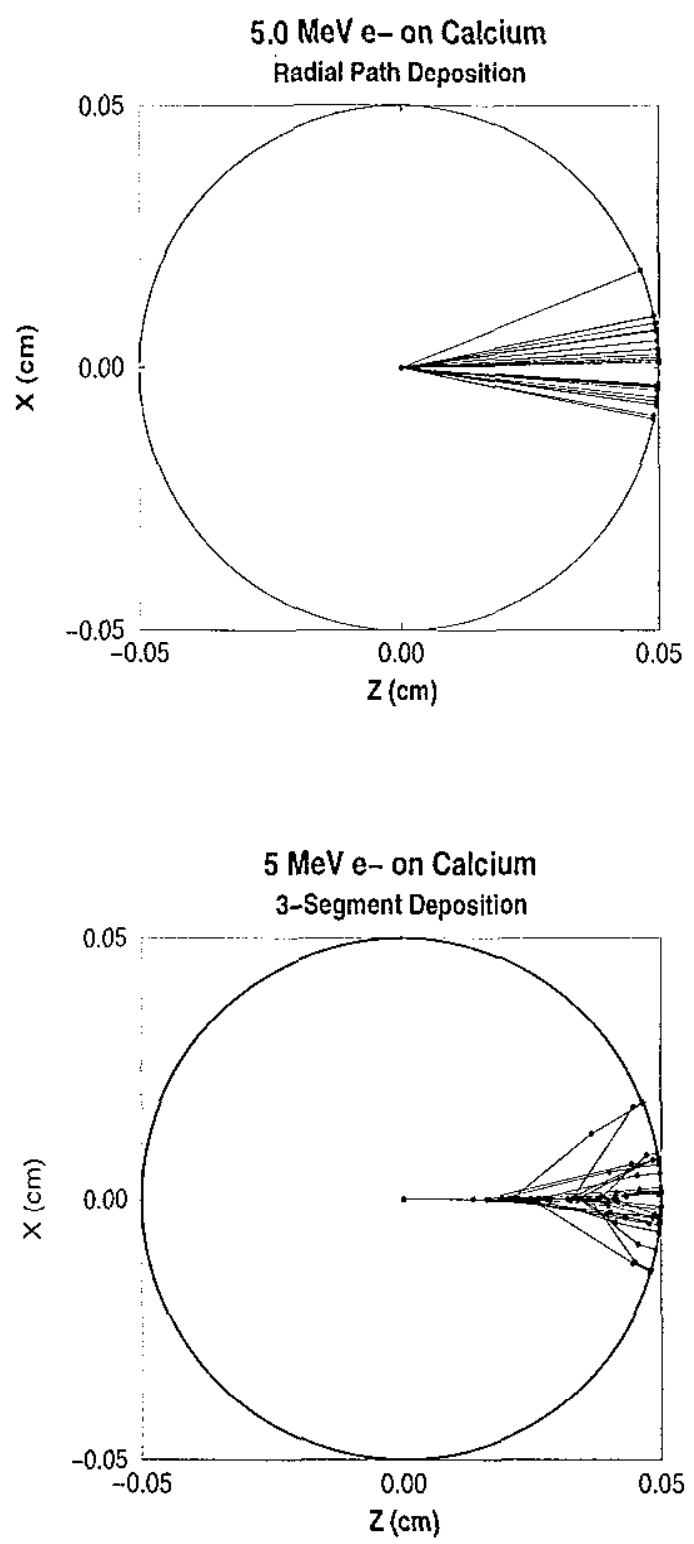

Figure 36: A comparison of paths through the kugel generated from the two algorithms tested. UPPER : $5.0 \mathrm{MeV}$ incidence; simple radial path deposition $\left(l_{1}=0\right.$; $\left.l_{3}=0\right)$. LOWER : $5.0 \mathrm{MeV}$ incidence; $\left(l_{1}=(0.3+0.6 \chi) R_{k u g} ; l_{3}=0.1 R_{k u g}\right)$. The difference in the depth dose curve that results was shown in Figure 33. 
the boundary. Although this may seem disconcerting (the kugel was generated for a homogeneous medium), any inconsistency is avoided. If the selected electron path through the kugel does not cross a zone of different material, transport proceeds as usual. If the selected path contains a zone of the other material, the deposition will once again abort.

For kugels with a relatively high probability of backscattering, the above logic could result in considerable "bouncing" between media, which in turn escalates the run time of the simulation. A possible remedy is to increase the size of $\Delta b$, the incremental "bump" into the new material. However, this type of shortcut must be exercised with caution since it could easily compromise the quality of the results.

\subsection{Tracking Secondary Particles}

\subsubsection{Treatment of Knock-on Electrons}

In Chapter 3 it was explained that the average number of knock-ons escaping the kugel per history is stored as a function of the primary exit energy bin. Knowing the mean number, the probability for the first $N$ knock-ons can be reconstructed with a Poisson distribution. Thus the number of knock-ons to track, $N_{k o n}$ is formally obtained by using a random number $\chi$ to set a limit, and summing terms in the Poisson distribution until

$$
\sum_{i=1}^{N_{k o n}} e^{-\bar{n}} \cdot \frac{\bar{n}^{i}}{i !} \geq \chi
$$

In practice, there is often little error and some time savings in doing a statistical interpolation between the integers bounding the mean value, i.e., using the remainder of the mean value as the probability for selecting the number to be 
$\operatorname{INT}(\bar{n})+1$, otherwise $\operatorname{INT}(\bar{n})$ is used.

For each escaping knock-on, an exit band must be sampled, along with its exit energy, trajectory and position. As stated in the previous chapter, these knock-on distributions are separate from the primary distributions. Once these quantities are known, they are put in a temporary knock-on bank for storage until the end of the primary's history. After the primary has either escaped the problem or been absorbed, the knock-ons are popped off the bank and tracked in exactly the same manner as the primary electrons.

\subsubsection{Treatment of Bremsstrahlung Photons}

The number of photons escaping the kugel must be determined in a manner similar to that of the knock-ons. However, there are a few differences since the code does not do photon transport. The distributions are not as tightly correlated, since there is only a single exit band over the entire kugel surface. Also, the photons are projected to the surface of the kugel along their initial trajectory. Thus any interaction that may have occurred between the birth point of the photon and the kugel surface is lost. However, since the mean free path of the photon is usually much longer than the kugel radius, this is a good approximation. After the photon phase space is sampled from the photon distributions, it is passed into a photon transport code. In the Peregrine implementation, each photon's phase space is written directly to Peregrine's main bank, where it is popped off and tracked between electron histories.

\subsection{Interface with the Parent Tracking Code}

The MRMC code has been written as a subroutine, assuming a parent code will provide the electron bank, define the transport mesh, and do any post-processing of the 
energy deposition. For physically valid results, the parent code must also have the ability to track photons. As has been discussed, MRMC produces bremsstrahlung photons which in turn generate Compton or photo-electrons, which are passed back to MRMC.

For all of the results shown in this work, the photon tracking was done by Peregrine Version 1.1. Peregrine also reads in the CT scan and assigns the materials. A different parent code which provides these capabilities could also be used, since contact with Peregrine occurs only through a few isolated subroutines which have access to Peregrine's global variables. 


\section{Chapter 5}

\section{Results of the MRMC Method}

\section{$5.1 \quad$ Introduction}

The Macro Response Monte Carlo Method was implemented in the form of a series of subroutines called by the Peregrine Version 1 code. The following test problems involve calculating the dose as a function of depth in a phantom, because that is the most relevant capability for the intended application. They stress two of the most important algorithms in MRMC - the energy deposition algorithm for spreading energy across several zones (typically 5-10), and the boundary crossing algorithm.

Three codes were used for comparison with the MRMC results: MCNP4b [13], EGS4[41] and the standard class 2 condensed history electron package inside of Peregrine version $1[30]^{1}$.

Several metrics were used to assess the agreement of each of the families of curves. First the total deposition to the medium, $E_{d e p}$, was compared. Secondly, the magnitude and location (x-coordinate) of the point of maximum dose, $D_{\max }$, was obtained. Finally, the practical range, $R_{p}$, was estimated for each curve by extrapolating from the straight descending portion of the curve to the bremsstrahlung tail. These quantities are summarized in a table for each phantom.

The standard deviations of the mean of the energy deposited for MCNP, EGS4 and Peregrine were less than $1 \%$ in the high dose regions (the upper $80 \%$ of the

\footnotetext{
${ }^{1}$ Other versions of Peregrine are under development.
} 
dose range) for all of the curves. The reported standard deviations with Peregrine running the MRMC electron transport package were also less than $1 \%$ in this region, however, the real statistical error in the MRMC global calculation results is difficult to quantify. This is because it is a result of both the number of histories used to generate the local PDFs and the number of historics used in the global calculation. The accuracy of the binning of the PDFs in the local code would also need to be folded into a formal error analysis. Error analysis in Local-to-Global methods is an area that requires further study.

\subsection{Test Problem 1: Infinite Medium}

The source for the infinite medium phantom is an embedded point source at $\mathrm{x}=$ $0.2 \mathrm{~cm}$ emitting electrons along the $\hat{x}$-direction. It was embedded primarily for analysis of the backscatter peak, which can be seen at the beginning of each curve. (External sources can be handled in MRMC with the usual boundary crossing techniques described in Chapter 4.) The size of phantom is $2 \mathrm{~cm} \times 2 \mathrm{~cm} \times 2 \mathrm{~cm}$. It contains 200 zones in the $\mathrm{x}$ direction and 1 zone in the $\mathrm{y}$ and $\mathrm{z}$ direction. This geometry is shown in Figure 37.

The depth dose curves that resulted from transport through this phantom for MCNP, Peregrine and MRMC at an incident energy of $4.9 \mathrm{MeV}$ are shown in Figure 38. A summary of the curve metrics is shown in Table 38. The three curves are quite close overall. The discrepancy in energy deposition between all three codes is less than $1 \%$. MRMC predicts a $D_{\text {max }}$ which is $2.5 \%$ lower than that of MCNP's $D_{\text {max }}$, and $1.7 \%$ lower than Peregrine's $D_{\text {max }}$. Peregrine and MRMC show the location of $\mathrm{D}_{\max }$ to be within the same $0.1 \mathrm{~mm}$ zone, while MCNP predicts it occurs $0.3 \mathrm{~mm}$ downstream. The practical range for the MRMC curve is $1.3 \%$ shorter than than of that Peregrine and $1.9 \%$ shorter than that of the MCNP curve. 


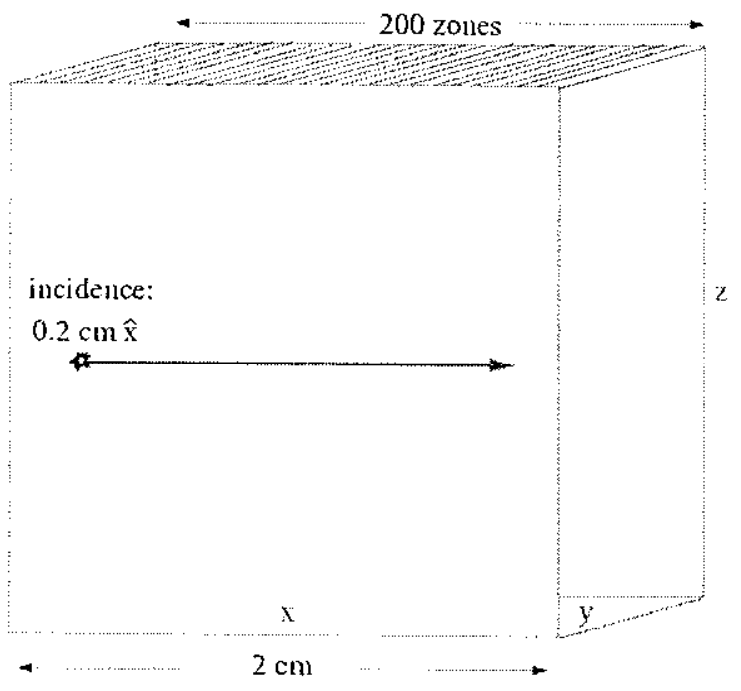

Figure 37: An illustration of the infinite medium phantom.

Table 10: A comparison of infinite calcium depth dose parameters for $4.9 \mathrm{MeV}$ electrons.

\begin{tabular}{|c|c|c|c|c|}
\hline Code & $\mathrm{E}_{\text {dep }}(\mathrm{MeV})$ & $\mathrm{D}_{\max }(\mathrm{mGy} / 100 \mathrm{k})$ & $\mathrm{x}\left(\mathrm{D}_{\max }\right)(\mathrm{cm})$ & $\mathrm{R}_{p}(\mathrm{~cm})$ \\
\hline \hline MCNP4B & 4.550 & 0.120 & 0.71 & 1.59 \\
\hline Peregrine v1 & 4.541 & 0.121 & 0.68 & 1.58 \\
\hline MRMC & 4.507 & 0.123 & 0.68 & 1.56 \\
\hline
\end{tabular}




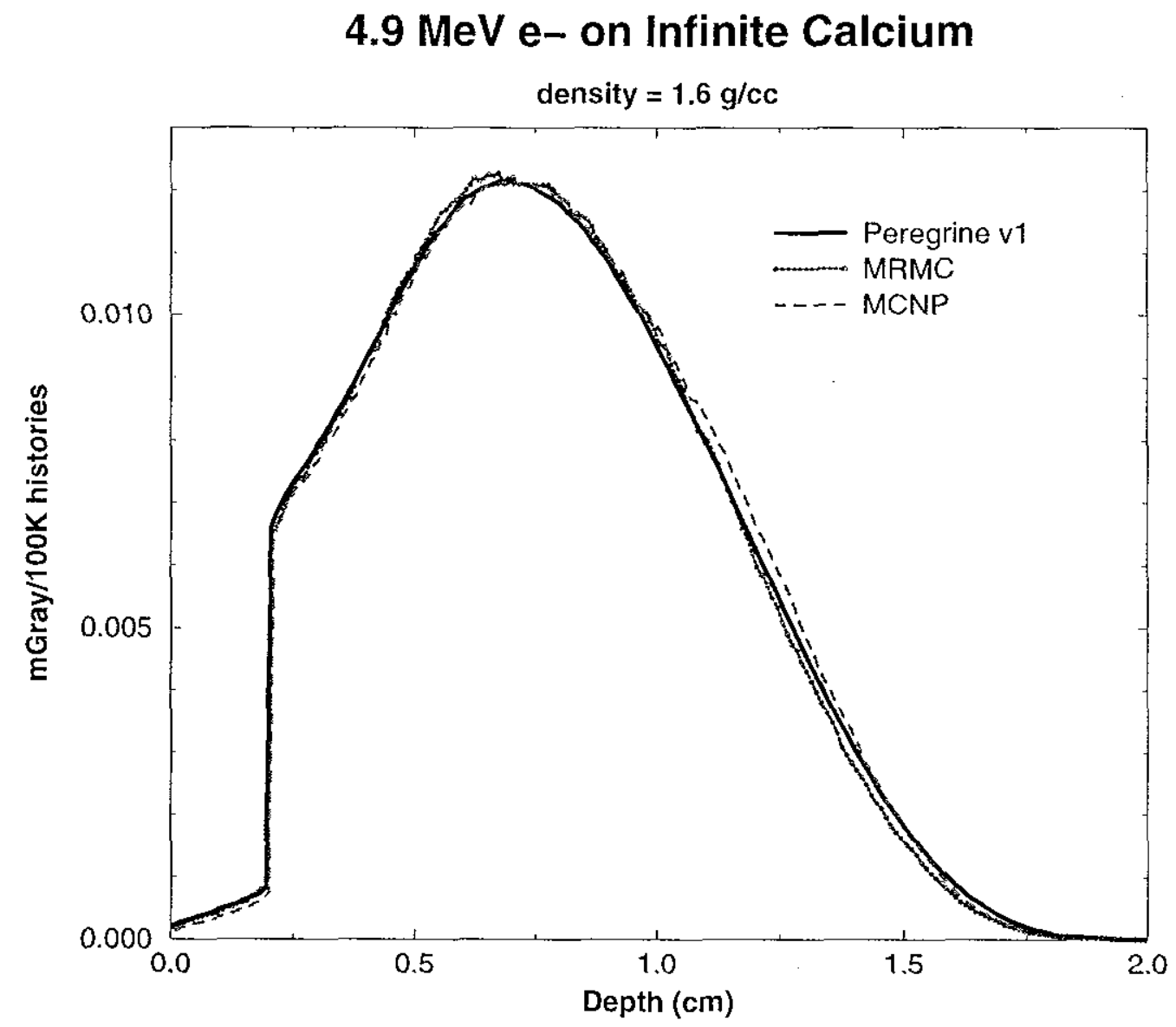

Figure 38: A depth dose comparison in an infinite calcium phantom for $4.9 \mathrm{MeV}$ electrons. A comparison of parameters derived from these curves can be found in Table 10. All runs used 100k incident histories. 


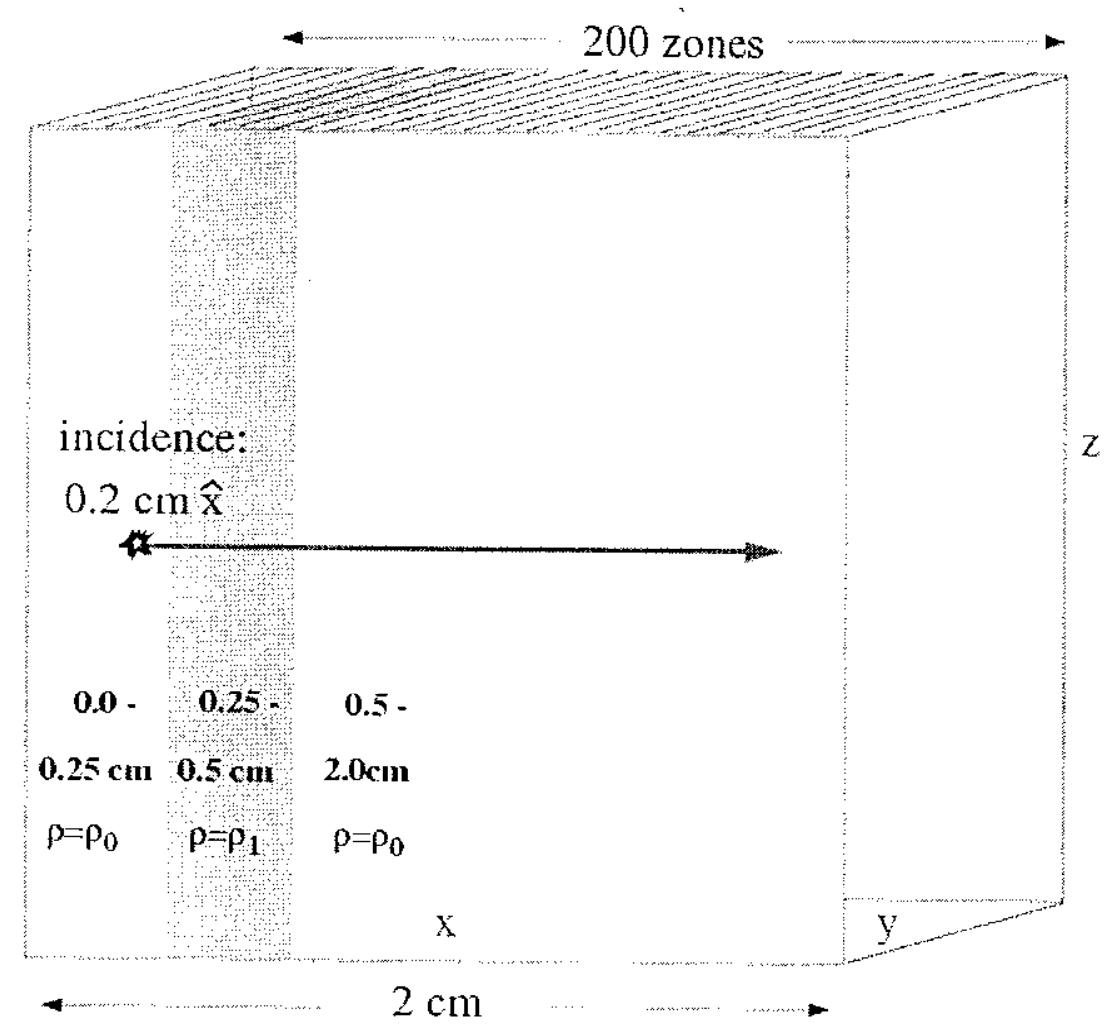

Figure 39: An illustration of the single-inhomogeneity phantom.

\subsection{Test Problem 2: Single Density Inhomogene- ity}

This phantom has a density inhomogeneity from a distance of $0.25 \mathrm{~cm}$ to $0.5 \mathrm{~cm}$ in the phantom, while the rest of the phantom has the density at its nominal value, which is $1.6 \mathrm{~g} / \mathrm{cm}^{2}$ for calcium. This is shown in Figure 39. The source is an embedded point source at $\mathrm{x}=0.2 \mathrm{~cm}$ emitting electrons along the $\hat{x}$-direction. The size of phantom is $2 \mathrm{~cm} \times 2 \mathrm{~cm} \times 2 \mathrm{~cm}$. It contains 200 zones in the $\mathrm{x}$ direction and 1 zone in the $\mathrm{y}$ and $\mathrm{z}$ direction; each transport zone is $0.01 \mathrm{~cm}$ in the $\hat{x}$-direction. 
Table 11: A comparison of low-density inhomogeneity calcium depth dose parameters for $4.9 \mathrm{MeV}$.

\begin{tabular}{|c|c|c|c|c|}
\hline Code & $\mathrm{E}_{\text {dep }}(\mathrm{MeV})$ & $\mathrm{D}_{\text {max }}(\mathrm{mGy} / 100 \mathrm{k})$ & $\mathrm{x}\left(\mathrm{D}_{\max }\right)(\mathrm{cm})$ & $\mathrm{R}_{p}(\mathrm{~cm})$ \\
\hline \hline EGS4 & 4.461 & 0.0119 & 0.84 & 1.73 \\
\hline Peregrine v1 & 4.481 & 0.0120 & 0.86 & 1.70 \\
\hline MRMC & 4.457 & 0.0121 & 0.83 & 1.67 \\
\hline
\end{tabular}

\subsubsection{Low Density}

The density inhomogeneity was assigned to half the nominal value, which is 0.8 $\mathrm{g} / \mathrm{cm}^{3}$ for calcium, in this test. The depth dose curves that resulted from transport through this phantom for EGS4, Peregrine and MRMC at an incident energy of 4.9 $\mathrm{MeV}$ are shown in Figure 40. A summary of the curve metrics is shown in Table 11. All three of the curves are quite close for total deposition and $\mathrm{D}_{\max }$. On the total deposition, MRMC is in agreement with EGS4 to less than 0.1\%; and in agreement with Peregrine to $0.5 \%$. On the $\mathrm{D}_{\max }$, MRMC is $1.7 \%$ higher than EGS4 and $0.8 \%$ higher than Peregrine. The practical range predicted by MRMC is $3.6 \%$ shorter than that predicted by EGS4, and 1.8\% shorter than that predicted by Peregrine.

\subsubsection{High Density}

The high-density spot phantom is identical to the one above, except the inhomogeneity has a density nearly twice the nominal value for calcium, $3.0 \mathrm{~g} / \mathrm{cm}^{3}$. The depth dose curves that resulted from transport through this phantom for EGS4, Peregrine and MRMC at an incident energy of $4.9 \mathrm{MeV}$ are shown in Figure 41. A summary of the curve metrics is shown in Table 12. The total energy deposition is in agreement to about $1 \%$ for all three codes and the location of $D_{\max }$ is within 1 


\subsection{MeV e- on 2-density Calcium}

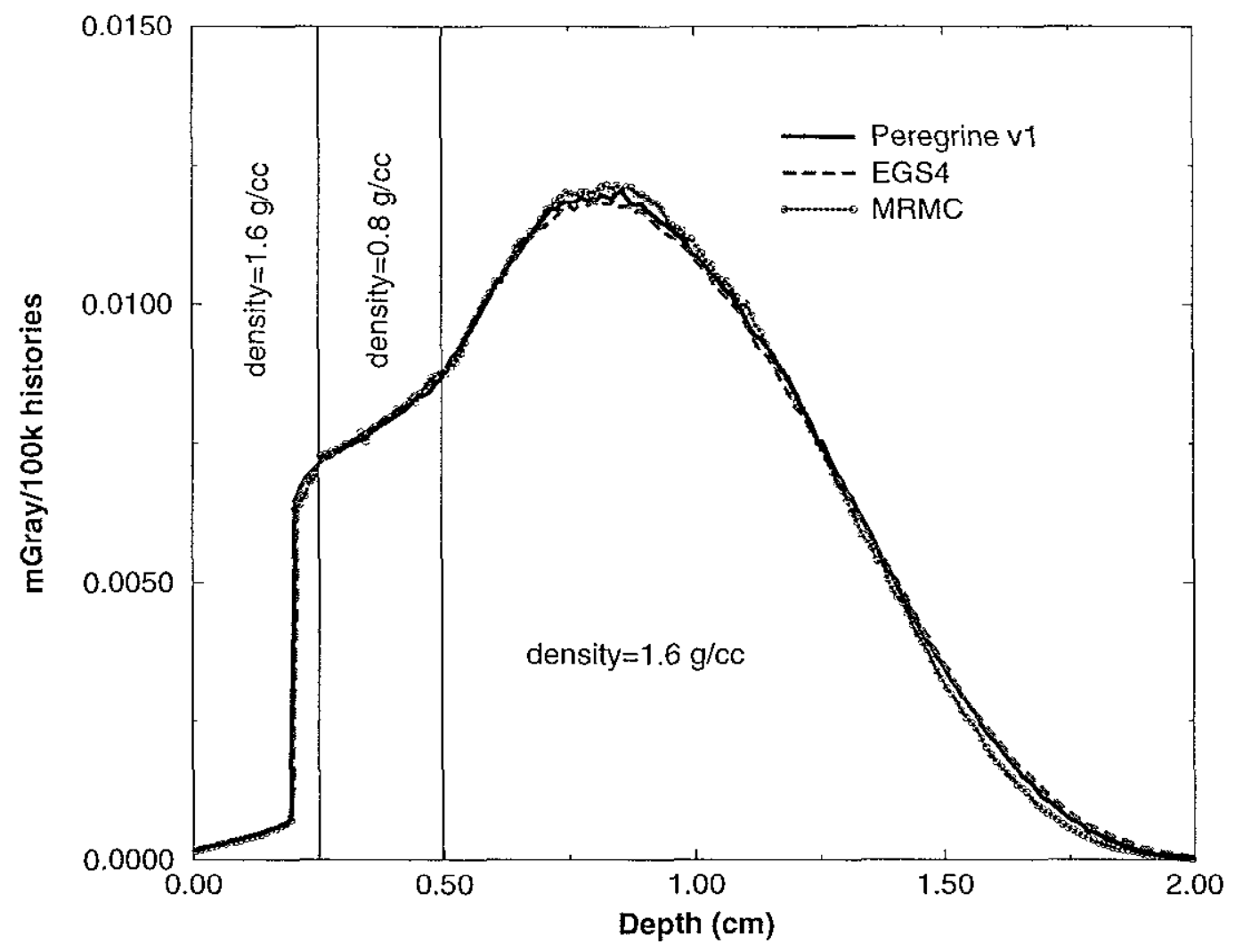

Figure 40: Calcium phantom with a low-density inhomogeneity depth dose comparison for $4.9 \mathrm{MeV}$ electrons. A comparison of parameters derived from these curves can be seen in Table 11. All runs used 100k incident histories. 
Table 12: A comparison of high-density inhomogeneity calcium depth dose parameters for $4.9 \mathrm{MeV}$.

\begin{tabular}{|c|c|c|c|c|}
\hline Code & $\mathrm{E}_{\text {dep }}(\mathrm{MeV})$ & $\mathrm{D}_{\max }(\mathrm{mGy} / 100 \mathrm{k})$ & $\mathrm{x}\left(\mathrm{D}_{\max }\right)(\mathrm{cm})$ & $\mathrm{R}_{p}(\mathrm{~cm})$ \\
\hline EGS4 & 4.615 & 0.0123 & 0.49 & 1.38 \\
\hline Peregrine v1 & 4.625 & 0.0128 & 0.50 & 1.37 \\
\hline MRMC & 4.574 & 0.0127 & 0.50 & 1.35 \\
\hline
\end{tabular}

zone. The magnitude of $\mathrm{D}_{\max }$ for MRMC is $3.2 \%$ greater than that for EGS4, but $0.8 \%$ less than that of Peregrine. The deviation between the codes in this quantity reflects the fact that the interface boundary is almost precisely at the location of $\mathrm{D}_{\max }$. The practical range predicted by MRMC is $1.5 \%$ short of that predicted by Peregrine and $2.2 \%$ short of that predicted by EGS4.

\subsection{Test Problem 3: "Riblet" Phantom}

This phantom has a series of thin inhomogeneities having a density of $3.0 \mathrm{~g} / \mathrm{cm}^{2}$. while the rest of the phantom has the density at its nominal value. It is pictured in Figure 42. The source is an embedded point source at $\mathrm{x}=0.2 \mathrm{~cm}$ emitting electrons along the $\hat{x}$-direction. The size of phantom is $2 \mathrm{~cm} \times 2 \mathrm{~cm} \times 2 \mathrm{~cm}$. It contains 200 zones in the $\mathrm{x}$ direction and 1 zone in the $\mathrm{y}$ and $\mathrm{z}$ direction; each transport zone is $0.01 \mathrm{~cm}$ in the $\hat{x}$-direction.

The depth dose curves that resulted from transport through this phantom for EGS4, Peregrine and MRMC at an incident energy of $4.9 \mathrm{MeV}$ are shown in Figure 43. A summary of the curve metrics is shown in Table 13. All the codes are within $1 \%$ on the total energy deposition. The magnitude of $D_{\max }$ in the MRMC curve is $0.8 \%$ higher than it is in the EGS4 curve, and lower by the same amount in 


\subsection{MeV e- on 2-density Calcium}

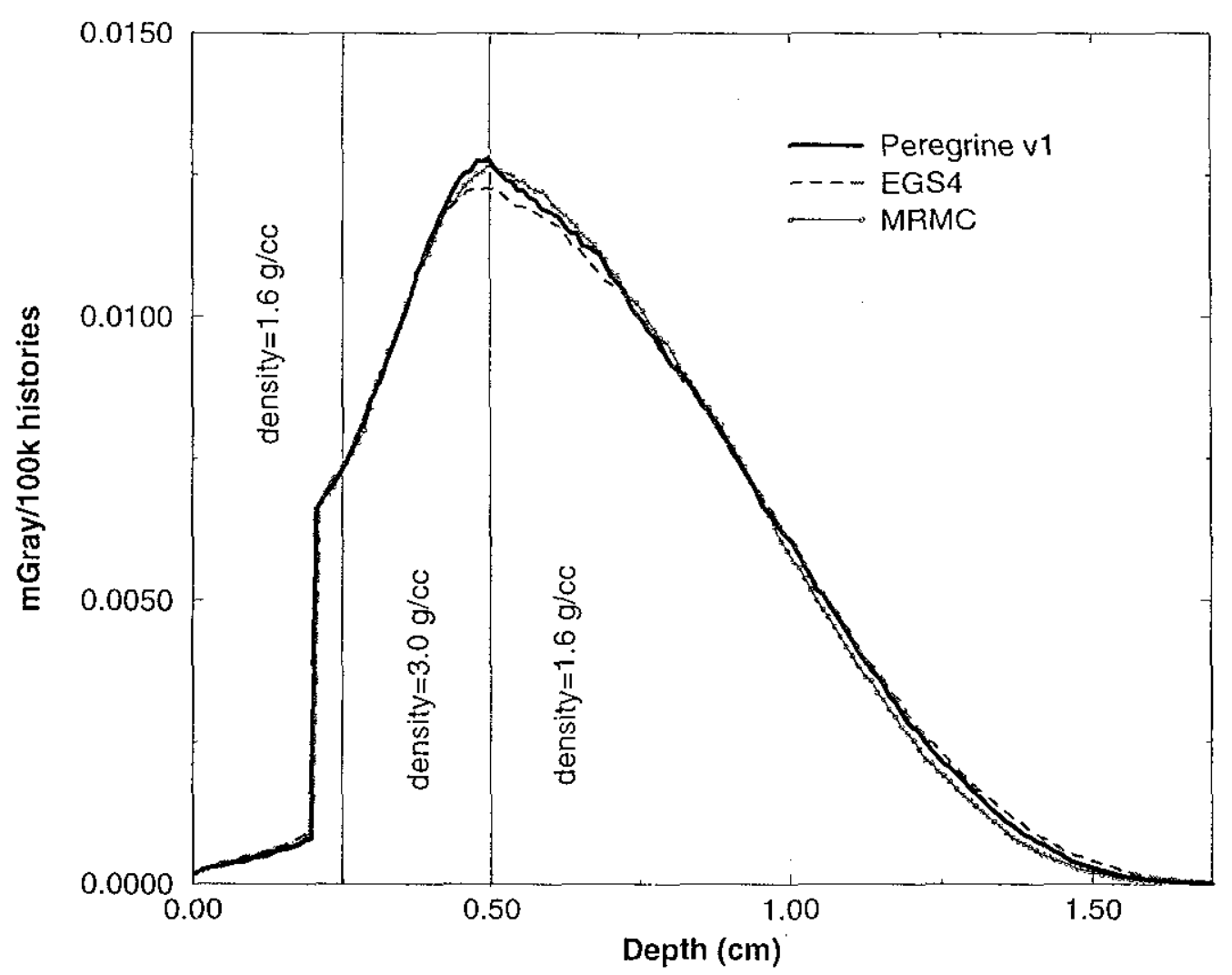

Figure 41: Calcium phantom with a high-density inhomogeneity depth dose comparison for $4.9 \mathrm{MeV}$ electrons. A comparison of parameters derived from these curves can be seen in Table 12. All runs used 100k incident electron histories. 


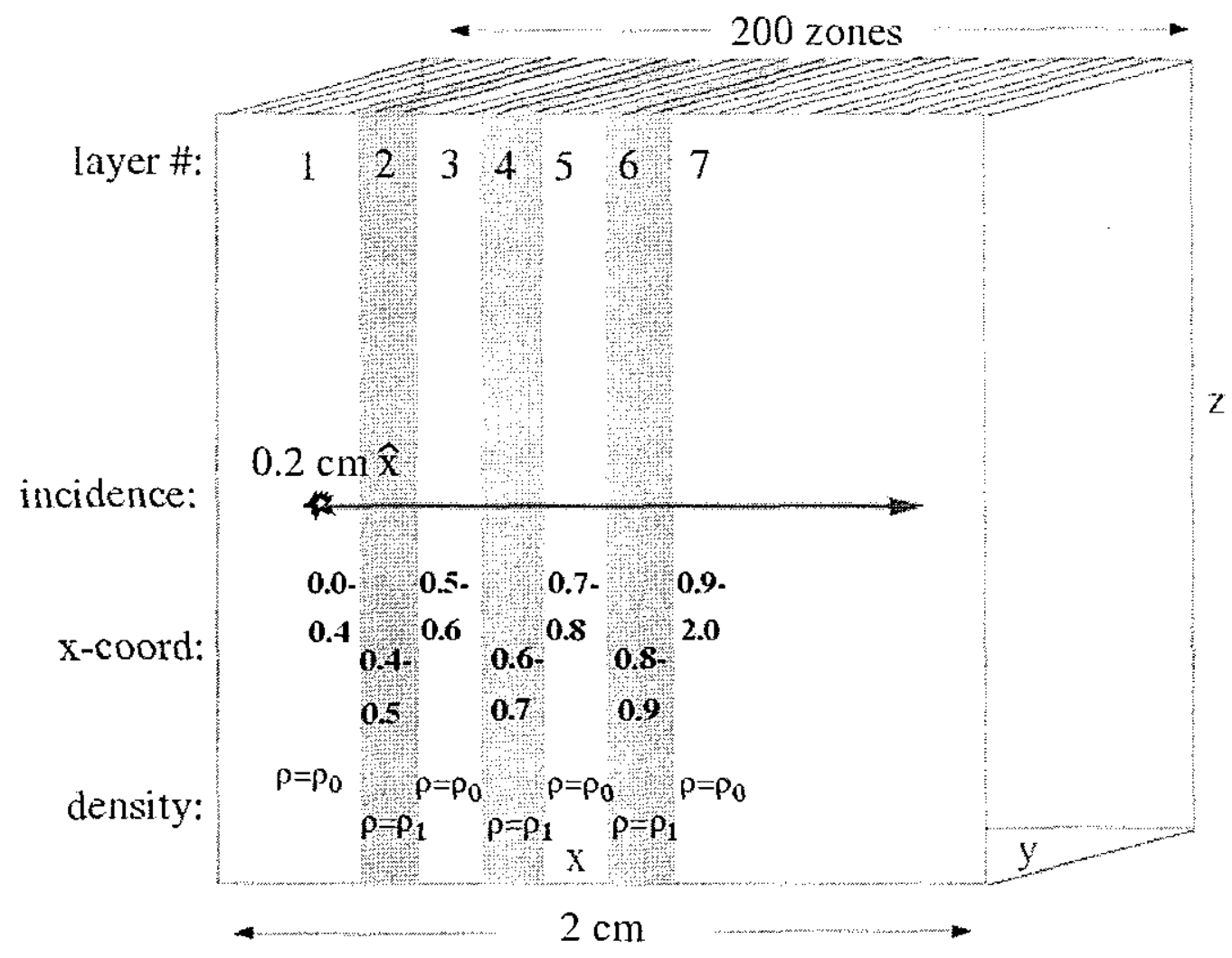

Figure 42: An illustration of the "riblet" phantom. 
Table 13: A comparison of "riblet" calcium phantom depth dose parameters for $4.9 \mathrm{MeV}$.

\begin{tabular}{|c|c|c|c|c|}
\hline Code & $\mathrm{E}_{\text {dep }}(\mathrm{MeV})$ & $\mathrm{D}_{\max }(\mathrm{mGy} / 100 \mathrm{k})$ & $\mathrm{x}\left(\mathrm{D}_{\max }\right)(\mathrm{cm})$ & $\mathrm{R}_{p}(\mathrm{~cm})$ \\
\hline \hline EGS4 & 4.620 & 0.0123 & 0.59 & 1.34 \\
\hline Peregrine v1 & 4.624 & 0.0125 & 0.62 & 1.32 \\
\hline MRMC & 4.575 & 0.0124 & 0.60 & 1.28 \\
\hline
\end{tabular}

the Peregrine curve. The location of $\mathrm{D}_{\max }$ on the MRMC curve is approximately midway between the EGS4 $\mathrm{D}_{\max }$ and that of Peregrine. There are some noticeable differences between the three codes, however. MRMC has difficulty particularly in the nominal-density regions which are "sandwiched" by the high-density regions, layers 3 and 5 in Figure 42. In these layers, MRMC is as much as $3.3 \%$ higher than EGS4 and Peregrine, which are in good agreement with each other. In layer 4, Peregrine is also as much as $3.3 \%$ higher than EGS4. The practical range of the MRMC curve was estimated to be shorter than that of EGS4 by about $4 \%$, and shorter by that of Peregrine by approximately $3 \%$. This quantity was somewhat more difficult to estimate on this phantom, since the inhomogeneities interfered with the declining slope of the curves.

\subsection{Summary of Global Test Problem Results}

Overall, the depth dose curves show that the MRMC method is performing reasonably well in the areas tested, as compared to several well-benchmarked, mature codes. The maximum excursion of any measured quantity was $4 \%$, which was the practical range of the MRMC curve in the riblet phantom compared to the EGS4 


\subsection{MeV e- on Riblet Phantom}

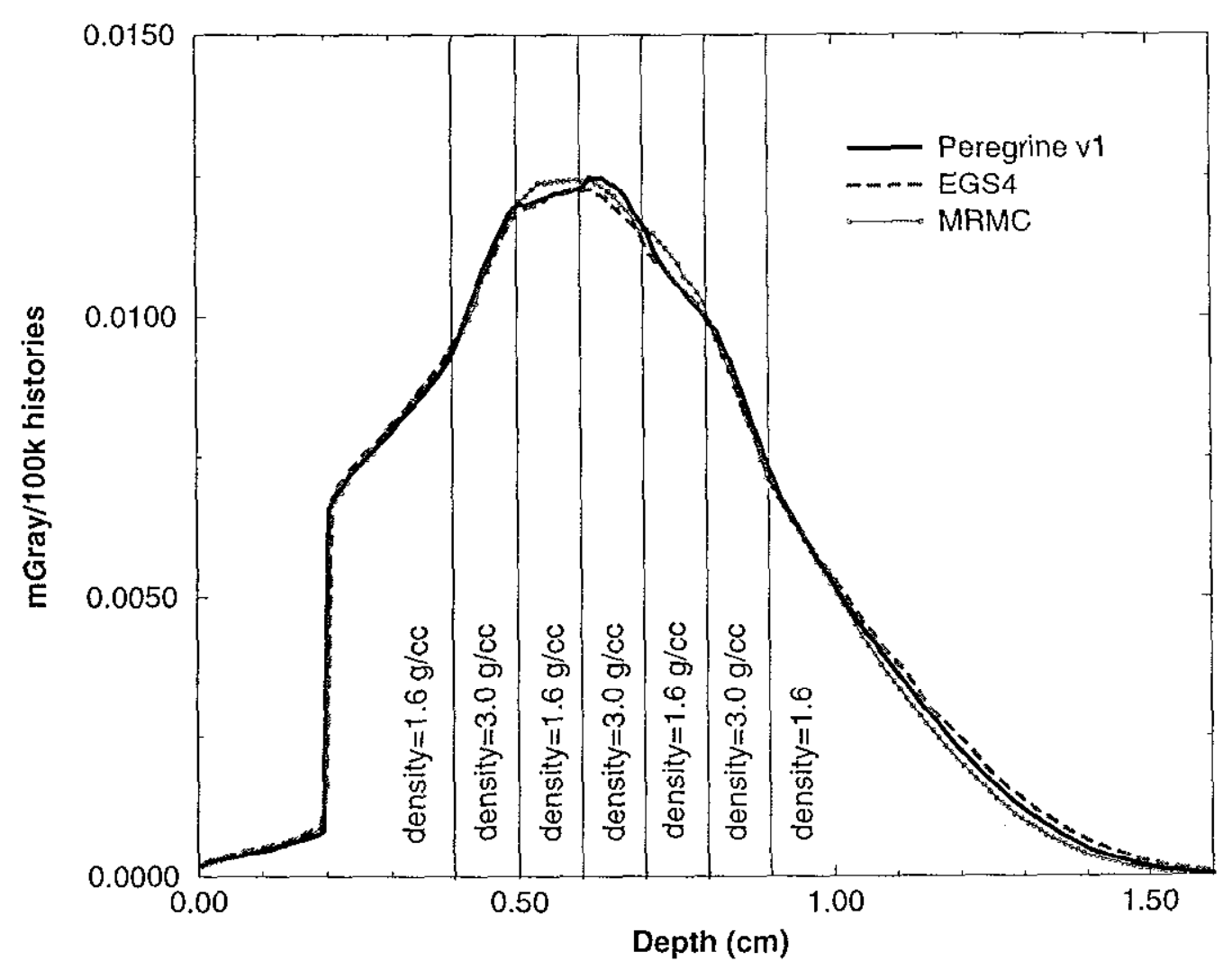

Figure 43: Calcium "riblet" phantom depth dose comparison for $4.9 \mathrm{MeV}$ electrons.

The parameters derived from these curves can be seen in Table 13. All runs used $100 \mathrm{k}$ incident electron histories. 
curve. The cases shown were intended to be stringent tests of the energy deposition and boundary crossing algorithms. The absolute dose deposited per particle agreed very well between all the codes in all the phantoms. The backscatter region is nearly identical for all the codes in all the phantoms. One minor point of concern is that MRMC demonstrates a small but consistant tendency to underestimate the practical range. Several cases indicate that the beginning of the depth dose divergence occurs at the point in the phantom where the electron has an energy of roughly $0.9 \mathrm{MeV}$ in calcium. It is possible that the energy spacing of kugel data sets in this energy region is slightly too coarse. This would be an important area for future studies.

\subsection{Timing for Global Test Problems}

The above studies indicate that the MRMC algorithm can cross 5-10 transport zones with a single kugel in a reliable manner. Although the MRMC code has not yet been optimized for speed, it is worthwhile to speculate on the significance of this for clinical problems. As stated in Chapter 1, equation 1.13, the time required for a Monte Carlo run is given by

$$
T_{\text {run }}=t_{\text {step }} * n_{\text {steps } / \text { history }} * n_{\text {histories }}
$$

The time savings of MRMC over Peregrine's condensed history package, $\eta$, running the same problem is given by

$$
\eta=\frac{T_{P G}}{T_{M R M C}}=\frac{\left(t_{\text {step }} * n_{\text {steps } / \text { history }}\right)_{P G}}{\left(t_{\text {step }} * n_{\text {steps } / \text { history }}\right)_{M R M C}}
$$

For the phantoms shown here, Peregrine with the MRMC electron transport package running the 3-segment energy deposition algorithm actually ran slower 
than the condensed history primarily due to the small scale of the phantoms. For the infinite calcium phantom, $\eta=0.526$, and $\left(n_{\text {steps/history }}\right)_{M R M C}=35.8$, while $\left(n_{\text {steps } / \text { history }}\right)_{P G}=157.8$. This indicates $t_{\text {step }}$ for MRMC is presently approximately $8.4^{*} t_{\text {step }}$ for Peregrine.

For clinical applications the phantom size would need to be scaled up by a factor of 10 , with transport zones of on the order of $1 \mathrm{~mm}$. The same scaling in kugel size would result in radii of $5 \mathrm{~mm}$, while Peregrine's step size would remain the same. The estimated time savings with the present MRMC code for clinical problems is thus approximately a factor of 5 . There is likely to be considerable memory swapping in these results to access the data from the large PDF arrays. Some cleaning up and tuning of the code for efficiency could probably enhance this number significantly. Results reported by other Local-to-Global algorithms, such as the MMC method introduced in Section 1.6.1, include times that are a factor of 15-20 faster than EGS4 [46]. 


\section{Chapter 6}

\section{Summary of the MRMC Method}

The main goal of this thesis was to prove the feasibility of basing electron depth dose calculations in a phantom on first-principles single scatter physics, in an amount of time that is equal to or better than current electron Monte Carlo methods.

Single scatter Monte Carlo has long been recognized as the most accurate way to perform electron transport, but is prohibitively slow for most simulations, due to the overwhelming number of electron interactions in media. The MRMC method achieves run times that are on the order of conventional electron transport methods such as condensed history, with the potential to be much faster. This is possible because MRMC is a Local-to-Global method, meaning the problem is broken down into two separate calculations. The first stage is a local, in this case, single scatter calculation, which generates probability distribution functions (PDFs) to describe the electron's energy, position and trajectory after leaving the local geometry. The second stage is a global calculation, which in this thesis, consists of depth dose calculations in simple phantoms with density inhomogeneities. Ultimately this stage could be a clinical radiation therapy treatment planning calculation.

A series of details were pieced together in this process.

First a family of analog Monte Carlo codes, CREEP, were created by relying on the Evaluated Electron Data Library at LLNL for cross sections. CREEP was benchmarked against experiments and other codes for backscatter percentages, reflected energy spectrum, energy deposition in various media as a function of 
depth. All comparisons were favorable, as can be seen in Chapter 2.

Next a version of CREEP was built to serve as the MRMC local code, in spherical "kugel" geometry. A systematic method of quantifying the variables describing the primary electron's exit state was decided upon, and extended to the secondary electrons and photons. A binning structure was devised for these quantities. First the kugel was divided into four surface bands, which were preferentially spaced in the forward direction, each band keeping separate distributions. Each distribution was further broken down into 100 equally spaced bins. Thus a "kugel data set" was generated with each run of the code, resulting in about $160 \mathrm{kB}$ of distributions.

The next step involved generating many kugel data sets, to put together a "kugel library" of results. A series of CREEP runs was performed for a variety of incident energies (from $0.25 \mathrm{MeV}$ to $8 \mathrm{MeV}$ ), step sizes $(0.025 \mathrm{~cm}$ to $0.1 \mathrm{~cm}$ in radius) and material types, although only the calcium and carbon libraries were complete enough for testing with the global code. Several codes to build multiple kugels efficiently were tried.

Finally, a global stepping code was written, in which every step conformed to a local calculation in the kugel library. The global code navigates and samples from the appropriate data set of the kugel library, and keeps track of quantities of interest in the global geometry, such as energy deposited per transport zone. An energy deposition scheme was devised to share the deposition across several zones within a kugel which meets the boundary conditions of the electron's path through the kugel. This was necessary because there is no information in the data set about where the electron traveled within the kugel.

The stepping code was benchmarked as a series of subroutines inside of the Peregrine Monte Carlo code. It was compared to Peregrine's usual class II condensed history electron transport package, EGS4, and MCNP for depth dose in simple phantoms having density inhomogeneities. Since the kugels completed in the library were of relatively small size, the zoning of the phantoms was scaled 
down from a clinical size, so that the energy deposition algorithms for spreading dose across 5-10 zones per kugel could be tested. Most resulting depth dose calculations were within $1-2 \%$ of well-benchmarked codes, with one excursion to $4 \%$.

Most of these logical steps described in this thesis have details that could be improved upon, and some potential improvements are mentioned in Section 6.1. Nonetheless, this thesis shows that the concept of using single scatter-based physics in clinical calculations would not only be possible, but would likely be more efficient than current methods, provided large kugels were generated.

\subsection{Areas for Future Development}

Some work remains before MRMC can be used for radiation treatment planning calculations. Both the local and the global codes need additional capabilities and/or improvements. Additionally, the MRMC library needs to be expanded to include a large set of appropriate materials, energies and the kugel sizes.

\subsubsection{Local Code Additions}

- Compounds/ mixtures

In order to include materials of biological relevance, it is essential to develop the ability to simulate electron transport in compounds and mixtures reliably. Although a version of CREEP was built to address this problem, it requires further benchmarking. The density effect may require special treatment for kugels of high energy in compounds, since the density effect included in the EEDL is for elements. This code was especially slow, and efficiency enhancements could also make the benchmarking process much smoother. 
- Low-energy physics

CREEP, and by extension MRMC, could fill an extremely useful niche in low energy, small volume studies if its low-energy physics models were enhanced. More details on this topic were provided in Chapter 2. In particular, CREEP's binding energy is locally deposited, rather than simulating the atomic relaxation process and tracking the resultant Auger electrons and fluorescent photons. Although LLNL maintains a database that would address these issues (EADL), it has not been coupled to CREEP's transport. This is not an area of concern for radiotherapy directly, but having these abilities in the code would make a wider range of benchmarks possible, especially against codes like EGS4, which include some relaxation physics.

- Paths Through the Kugel

Ultimately it is necessary to consider storing PDFs for electron paths through the kugel. In order to obtain large gains in speed, large kugels must be used that will span many zones in the global transport stage. As was demonstrated in Chapter 4, the key to getting accurate results for dose deposition is to partition the kugel's energy deposit accurately across the zones. Studies are needed to examine and characterize the paths. One possibility is to store parameters that relate to each path as a whole, such as the length and deviation from the simple radial path at various points. Another possibility would be to partition the kugel's internal volume and keep tallies of the partitions hit by each history.

\subsubsection{MRMC Library Building and Extension}

- Materials

The MRMC library must include data sets for all biological materials, in addition to materials likely to be used in the gantry head and beam-shaping 
devices.

- Optimization of Number and Placement of PDF Bins

Currently, all PDFs for all energies and materials are treated the same with 100 equally placed bins. This method is satisfactory over the small size and energy range tested, however, when the library is extended to larger sizes, and a larger energy range it will be wasteful due to storing large areas of bins with few counts. It will also not resolve narrow distributions well enough. This is especially important at higher energies, where the PDFs are extremely forward peaked. With the present scheme for tallying exit energy, for example, almost all the electrons would end up in the highest bin, and there would be almost no counts at lower bins. The extrema for the bin placement could be based on stopping power calculation. In general a scheme with variable resolution is necessary.

\section{- Post-Processing of Kugel Data}

As can be seen in Appendix B, many of the PDFs that result from CREEP are noisy. This is especially true of the PDFs for bands which did not receive many events. The tallies for knock-on electrons are also especially noisy at those incident energies where few escape. It could be advantageous to employ a smoothing algorithm on these distributions. It may also be interesting to investigate alternatives for storing raw kugel data - like finding analytic representations for the distributions, which would decrease the memory requirements significantly. However, these tactics would have to be employed cautiously to avoid biasing the physics. 


\subsubsection{Global Code Enhancements}

- Energy deposition scheme

The energy deposition scheme in MRMC is likely to be the single biggest obstacle for achieving excellent depth dose results with very large kugels. More information is needed about how to spread the energy in a realistic way over many zones. One way to achieve this is to store information about the path in a PDF generated in the local calculation, as discussed above. Another alternative may be to start with a path that meets the known boundary conditions in the global code, then use a pathlength correction from condensed history scattering theories.

- Benchmarking

More depth dose curves with a larger variety of materials and energies are clearly necessary to test this method further. In addition, studies that explicitly examine the lateral scatter and backscatter in phantoms should be undertaken, since this method should show improvements over current clinical simulation methods in this regard.

- Error Analysis

In order to fully evaluate the benchmarks, it is necessary to quantify the error in the global calculation. This would require a propagation of uncertainities through the local code. A formal series of sensitivity studies to determine the importance of various parameters on the global calculation would be a useful starting point.

- Code Efficiency

The biggest gains in efficiency can probably be obtained by cleaning up and tuning the code, which was not written with this in mind. Further efficiency enhancement can bc obtained by pre-processing the transport mesh for kugel 
size, as seen in Neuenschwander's Macro Monte Carlo [44]. This eliminates the need to choose a kugel size during transport.

\subsection{Other Applications}

Aside from radiation treatment planning calculations in a phantom or patient volume, there are several other areas of potential application for all or part of the MRMC method.

- MRMC

The most promising application for this work may be in the characterization of the radiation source. It is likely to be both the most efficient and the most accurate way to transport electrons through the gantry head of the accelerator and the patient-specific beam-shaping devices. The energy deposition algorithm is not a factor in this application, since it is the transmitted energy that is of interest. The MRMC global calculation is not slowed down by dense, high-z materials, and it can simulate low probability events like backscatter, which are more important in these materials, more accurately than condensed history methods.

- CREEP

The CREEP code has the potential to be extremely useful for detailed electron physics simulations on a small scale, due to its extreme accuracy. This would include evaluation of new electron transport methods and scattering theories, modeling small ion chambers or other detectors, and possibly semiconductors on a small scale.

- Other Local-to-Global calculations

Other types of "dense" particle transport may also benefit from the Local-toGlobal concept. This includes any application where there are an extremely 
high number of interactions over the region of interest: such as proton transport; or, photons or neutrons which must travel through media where the mean free path is very small compared to the dimensions of the problem. Other types of particle transport would require separate local codes, but that capability exists for each of those listed. There no inherent restriction in the global MRMC transport to electrons; it is simply reading distribution functions without regard to what type of particle they represent. However, it is possible different particle types may require different types of distributions. 


\section{Appendix A}

\section{Further Details on Scattering}

\section{Theories}

\section{A.1 Moliere theory of Multiple Scattering}

The Moliere theory expresses the distribution of multiple scattering deflections as a unique function of a scaled angular variable, $\vartheta$, as is seen by

$$
F_{M} \vartheta d \vartheta=\vartheta d \vartheta\left[e^{\vartheta^{2}}+\frac{F^{(1)}(\vartheta)}{B}+\frac{F^{(2)}(\vartheta)}{B^{2}}+\cdots\right] .
$$

The relationship between the scaled angle $\vartheta$ and the true deflection angle, $\theta$ is given by the identity

$$
\vartheta=\frac{\theta}{\chi_{c}} \sqrt{B}
$$

where $\chi_{c}$ and $B$ are functions of particle energy and path length $l$. The expressions for $F^{(1)}$ and higher orders are obtained from the equation

$$
F^{(n)}(\vartheta)=\frac{1}{n !} \int_{0}^{\infty} y d y J_{0}(\vartheta, y) e^{\frac{-y^{2}}{4}}\left(\frac{y^{2}}{4} \ln \frac{y^{2}}{4}\right)^{n}
$$

where $J_{0}$ is a Bessel function of zeroth order. The values for each term inside the square braces in equation [A.1] ( $F^{(0)}$ through $F^{(2)}$, as well as higher terms) have been tabulated by Bethe [8], and Scott [53].

The screening angle, $\chi_{\alpha}$, is given by

$$
\chi_{\alpha}^{2}=\frac{6.8 \times 10^{-5} Z^{2}}{\tau(\tau+2)}\left[1.13+3.76\left(\frac{Z}{137 \beta}\right)^{2}\right]
$$


where $\tau$ is the kinetic energy in units of electron rest mass, $Z$ is atomic number, and $\beta$ is the magnitude of the particle velocity in units of the speed of light.

The parameter $\chi_{c}^{2}$ is the characteristic angle, which is related to the interaction probability in a step size and can be found by

$$
\chi_{c}^{2}=0.6009 \frac{Z^{2}}{A}\left[\frac{\tau+1}{\tau(\tau+2)}\right]^{2} s
$$

where $A$ is the atomic mass and $s$ is the step length.

The Moliere parametcr $B$ depends on the ratio of the characteristic angle $\chi_{c}$ to the screening angle $\chi_{\alpha}$, and is obtained iteratively from the transcendental equation

$$
B-\ln B=\ln \left(\frac{\chi_{c}}{\chi_{\alpha}}\right)^{2}+1-2 \gamma,
$$

where $\gamma$ is Euler's constant, $\gamma=0.577215 \ldots$.

\section{A.2 Goudsmit-Saunderson Model for Multiscat- ter}

The Goudsmit-Saunderson distribution takes the form of a Legendre series, where the $P_{\varepsilon}$ 's are the Legendre polynomials

$$
F_{G S}(\theta, s)=\sum_{\ell=0}^{\infty}\left(\ell+\frac{1}{2}\right) e^{-s G_{\ell}} P_{\ell}(\cos \theta),
$$

where $s$ is the step length (in $\mathrm{g} / \mathrm{cm}^{2}$ ). The coefficients $G_{\ell}$ are calculated by using the equation

$$
G_{\ell}=2 \pi \frac{N_{a}}{A} \int_{0}^{\pi}\left[1-P_{\ell}(\cos \theta)\right] \sigma(\theta) \sin \theta d \theta,
$$

where $N_{A}$ is Avogadro's number and $A$ is the atomic weight. To account for the energy loss of the electron along its track, the following substitution can be made in equation [A.7]

$$
s G_{\ell} \rightarrow \int_{T 1}^{T_{0}} \frac{G_{\ell}(T)}{S(T)} \rho d T
$$


where $T_{0}$ is the initial electron kinetic energy at the beginning of the step of length $s$, after which the electron has a kinetic energy of $T_{1}$. The quantity $\frac{S(T)}{\rho}$ is the mass stopping power of the medium in $\mathrm{MeV}-\mathrm{cm}^{2} / \mathrm{g}$. 


\section{Appendix B}

\section{Example Kugel PDFs}

The following pages show an example of the primary electron and knock-on probability distribution functions (PDFs) in a kugel data set for a $3 \mathrm{MeV}$ calcium kugel of radius $0.05 \mathrm{~cm}$, and density $1.6 \mathrm{~g} / \mathrm{cm}^{3}$. There were 500,000 incident electrons, each of which underwent an average of 2062 interactions. Over $99.8 \%$ of them were transmitted through the kugel. There were 363,548 knock-on electrons created over $10 \mathrm{keV}$, but only $3.3 \%$ escaped the kugel. Only the knock-ons which escaped are tallied. Details on all of the tallied quantities can be found in Chapter 3.

All histogram probabilities are presented here the same way they are stored in the MRMC library: they are normalized to the number of incident electrons and divided by the width of the bin. Multiplying by the bin width would yield the probability of selecting any value in the bin. 


\section{Band 1 Position Cosine (z/R)}

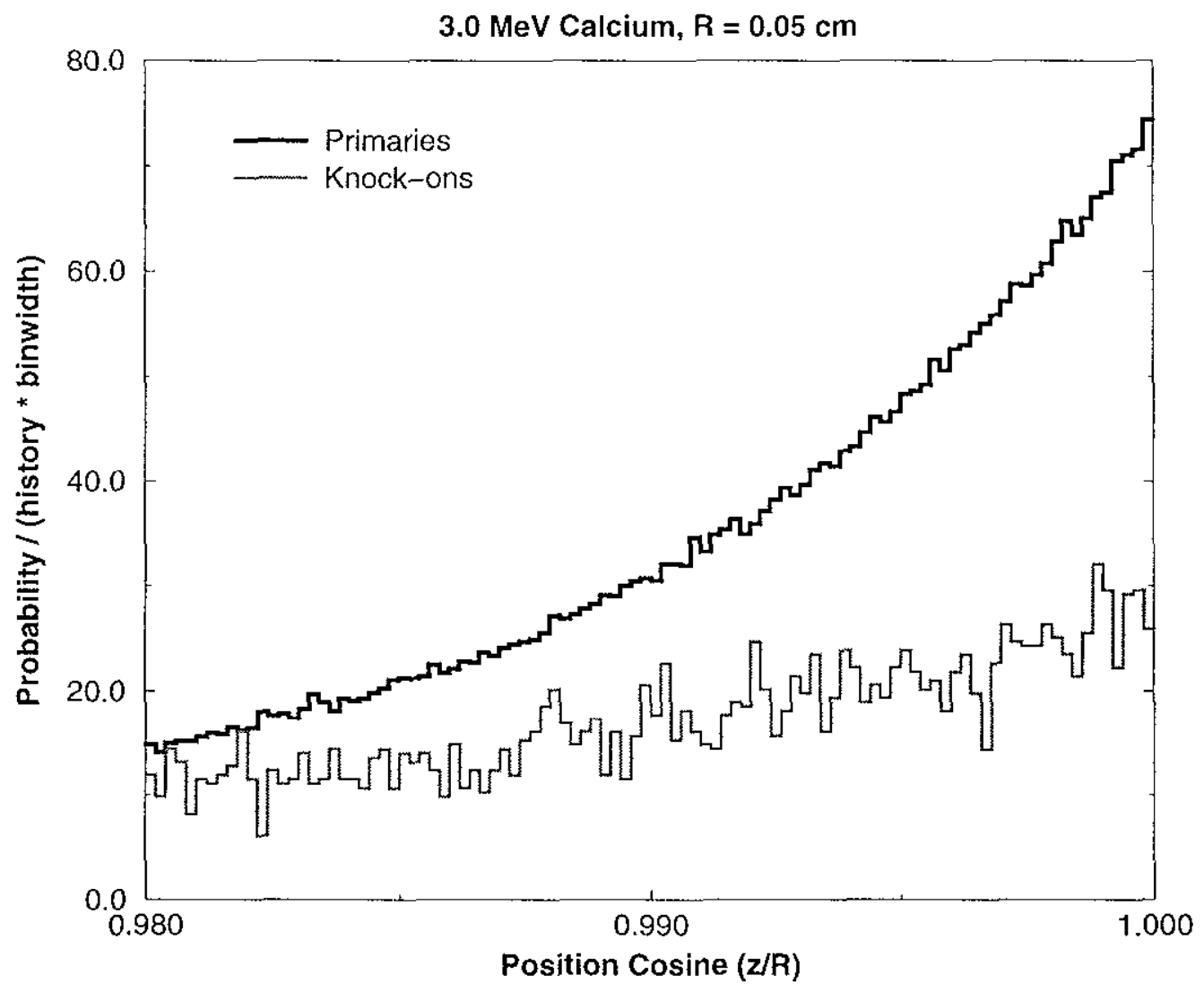

Figure 44: Exiting position cosines for primary electrons and escaping knock-ons from Band 1 of a $3 \mathrm{MeV}$ calcium kugel of radius $0.05 \mathrm{~cm}$. 


\section{Band 2 Position Cosine ( $\mathrm{z} / \mathrm{R})$}

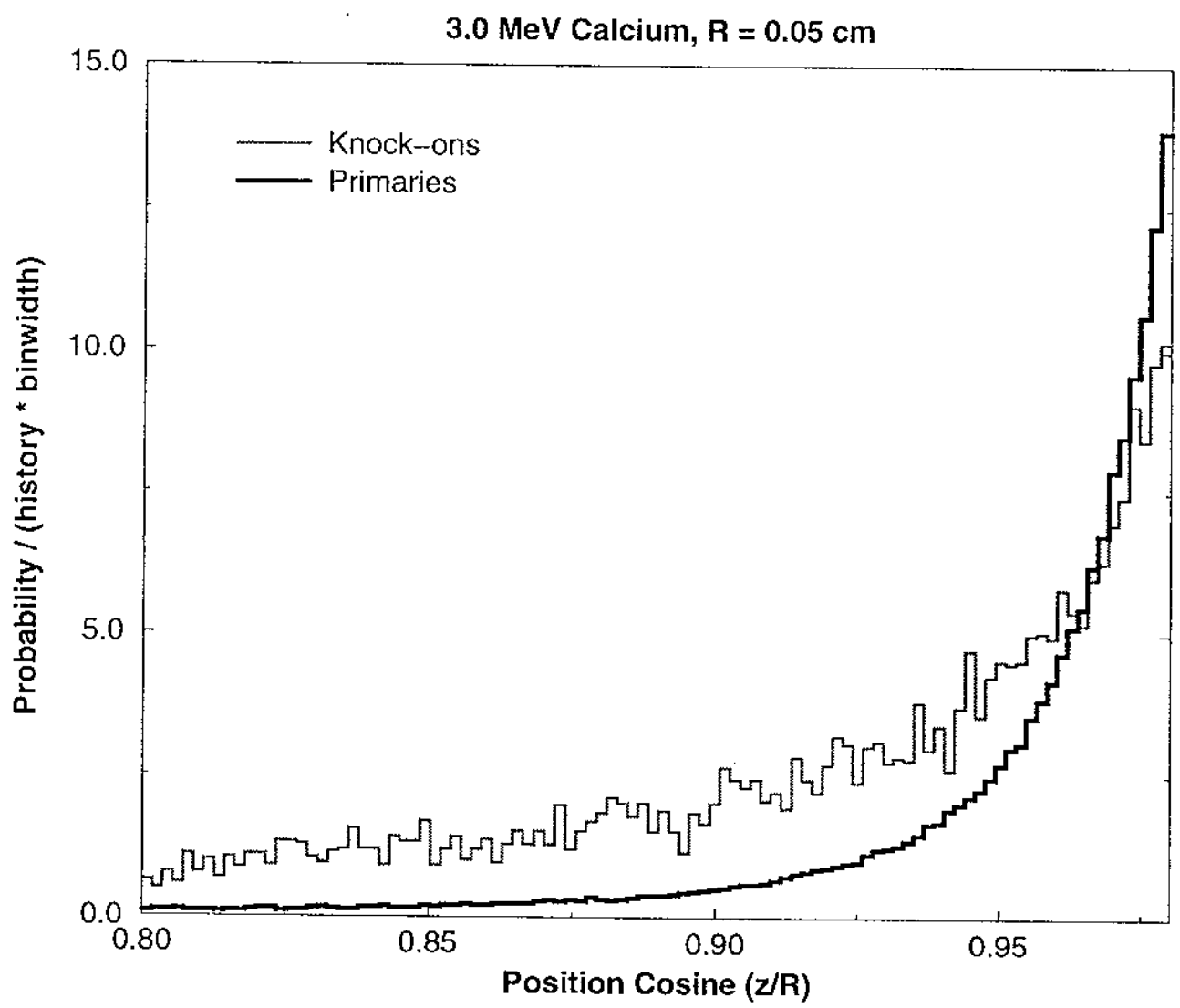

Figure 45: Exiting position cosines for primary electrons and escaping knock-ons from Band 2 of a $3 \mathrm{MeV}$ calcium kugel of radius $0.05 \mathrm{~cm}$. 


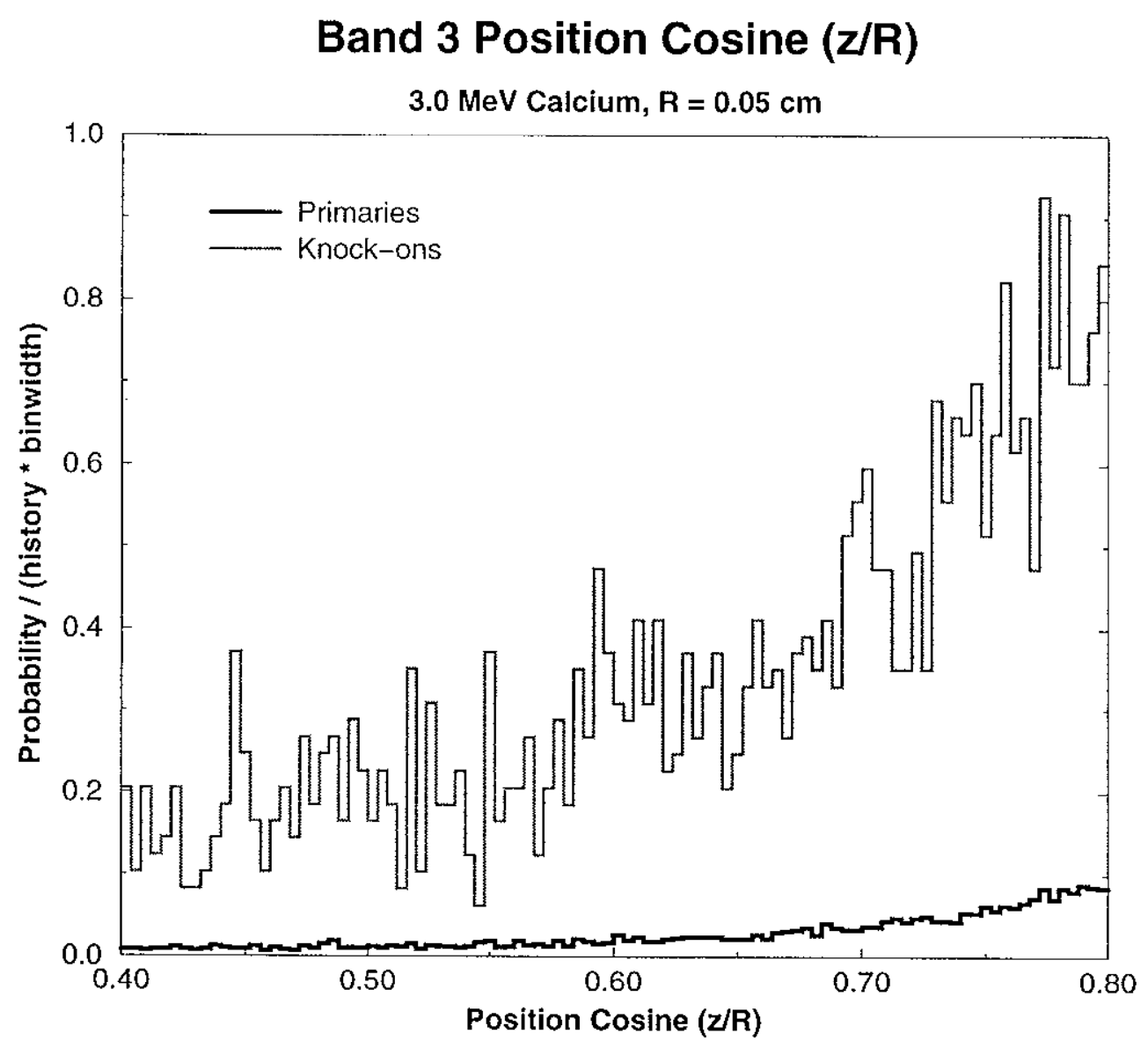

Figure 46: Exiting position cosines for primary electrons and escaping knock-ons from Band 3 of a $3 \mathrm{MeV}$ calcium kugel of radius $0.05 \mathrm{~cm}$. 


\section{Band 4 Position Cosine (z/R)}

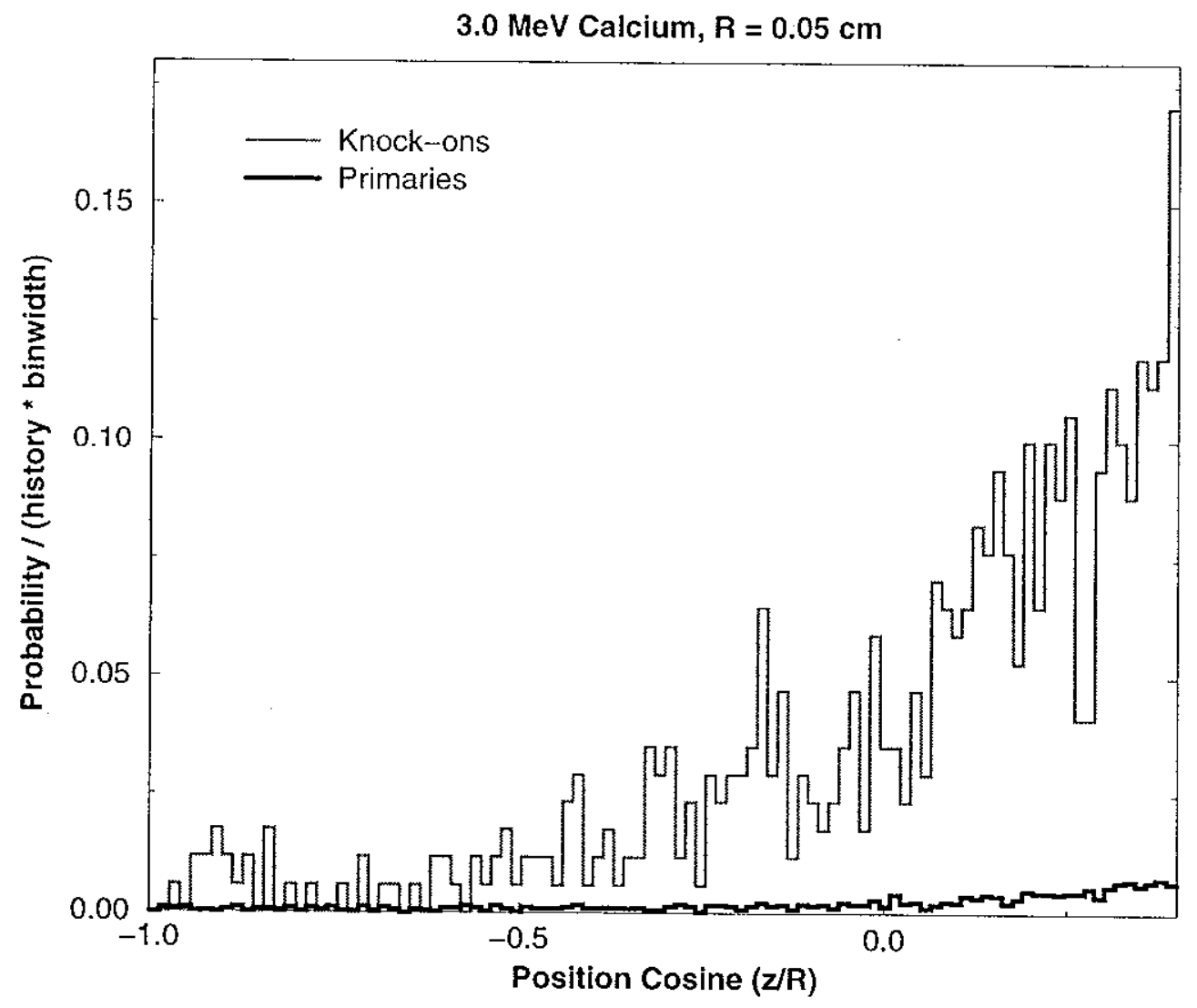

Figure 47: Exiting position cosines for primary electrons and escaping knock-ons from Band 4 of a $3 \mathrm{MeV}$ calcium kugel of radius $0.05 \mathrm{~cm}$. 


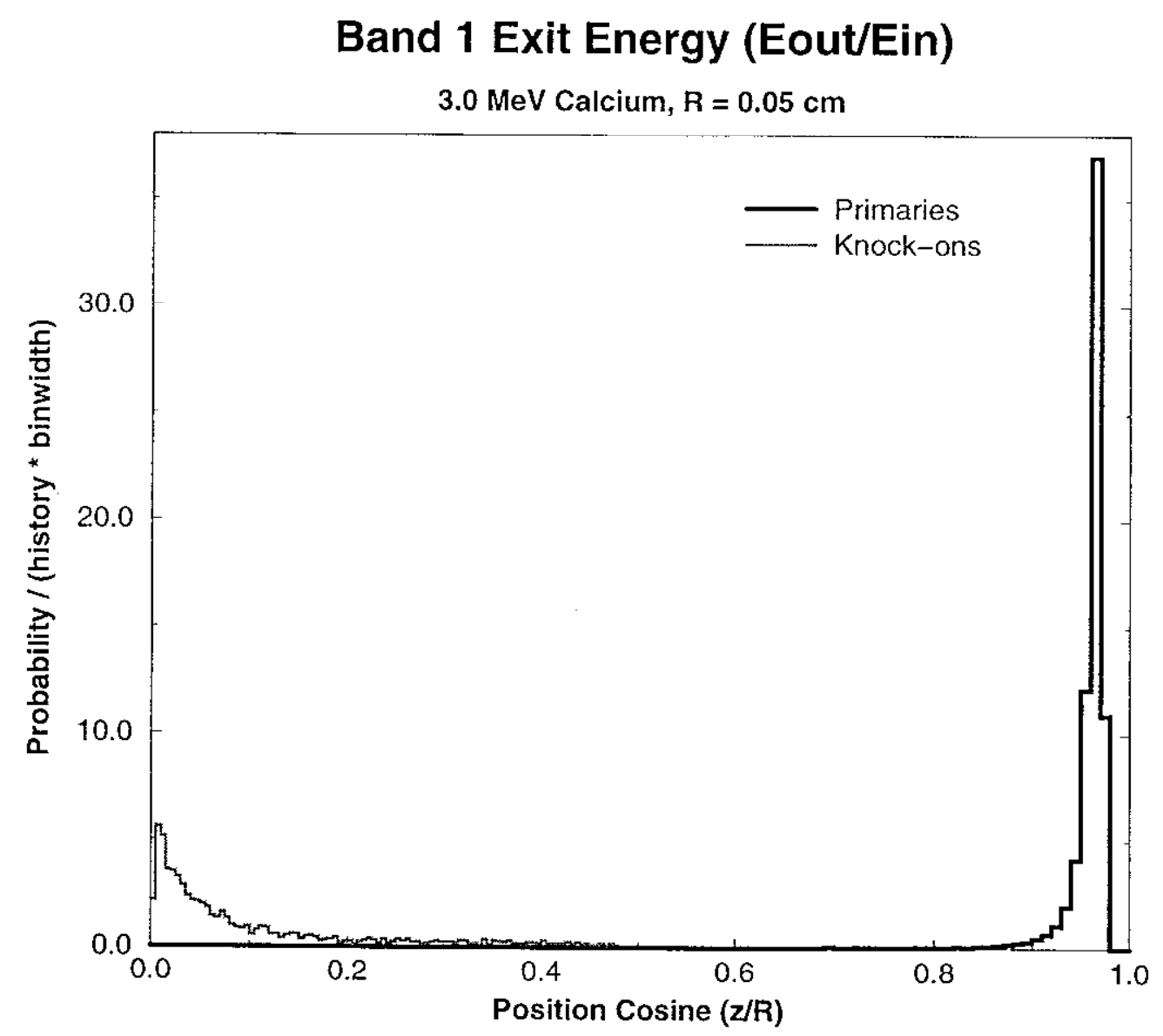

Figure 48: Exiting energy for primary electrons and escaping knock-ons from Band 1 of a $3 \mathrm{MeV}$ calcium kugel of radius $0.05 \mathrm{~cm}$. 


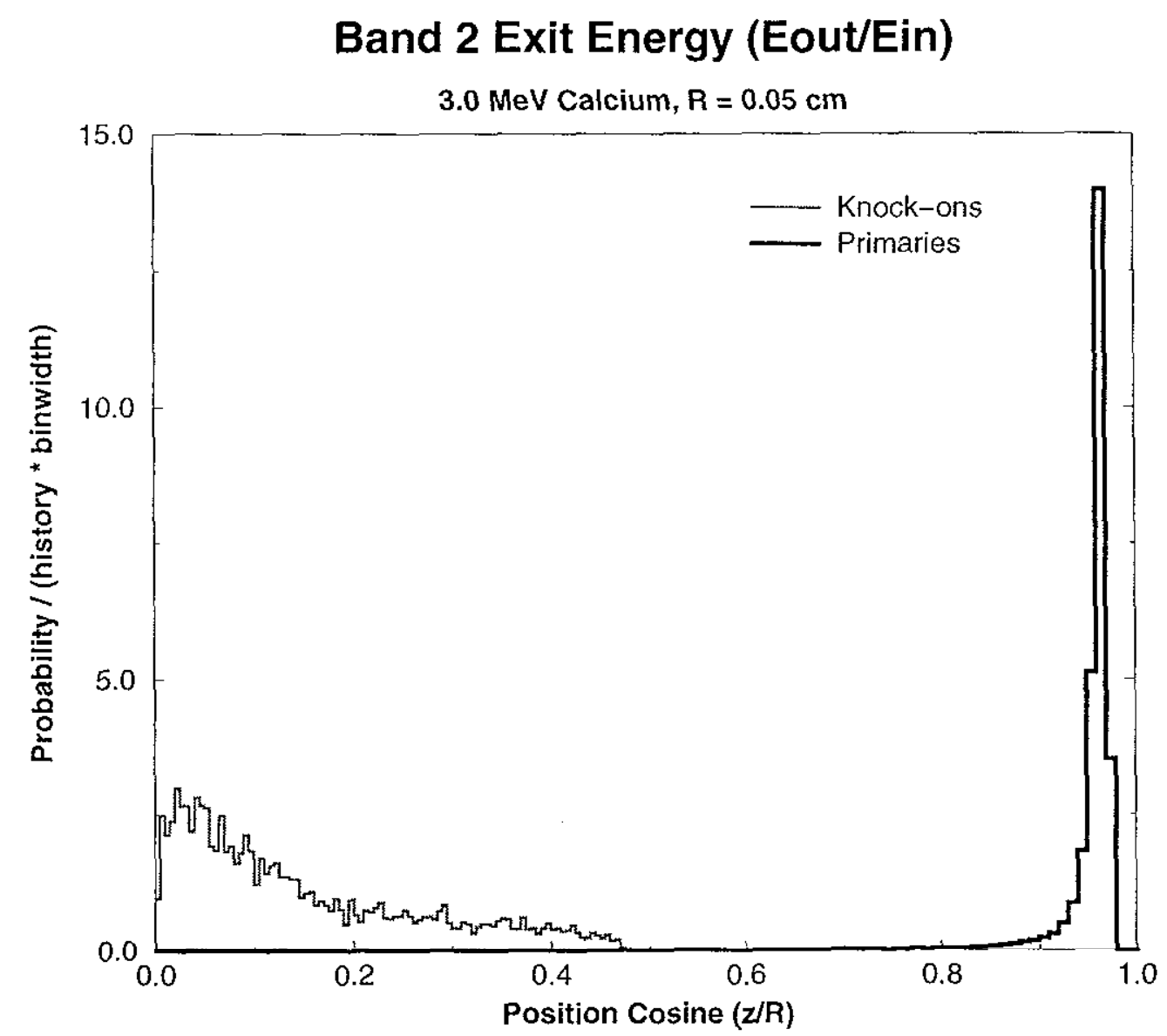

Figure 49: Exiting energy for primary electrons and escaping knock-ons from Band 2 of a $3 \mathrm{MeV}$ calcium kugel of radius $0.05 \mathrm{~cm}$. 


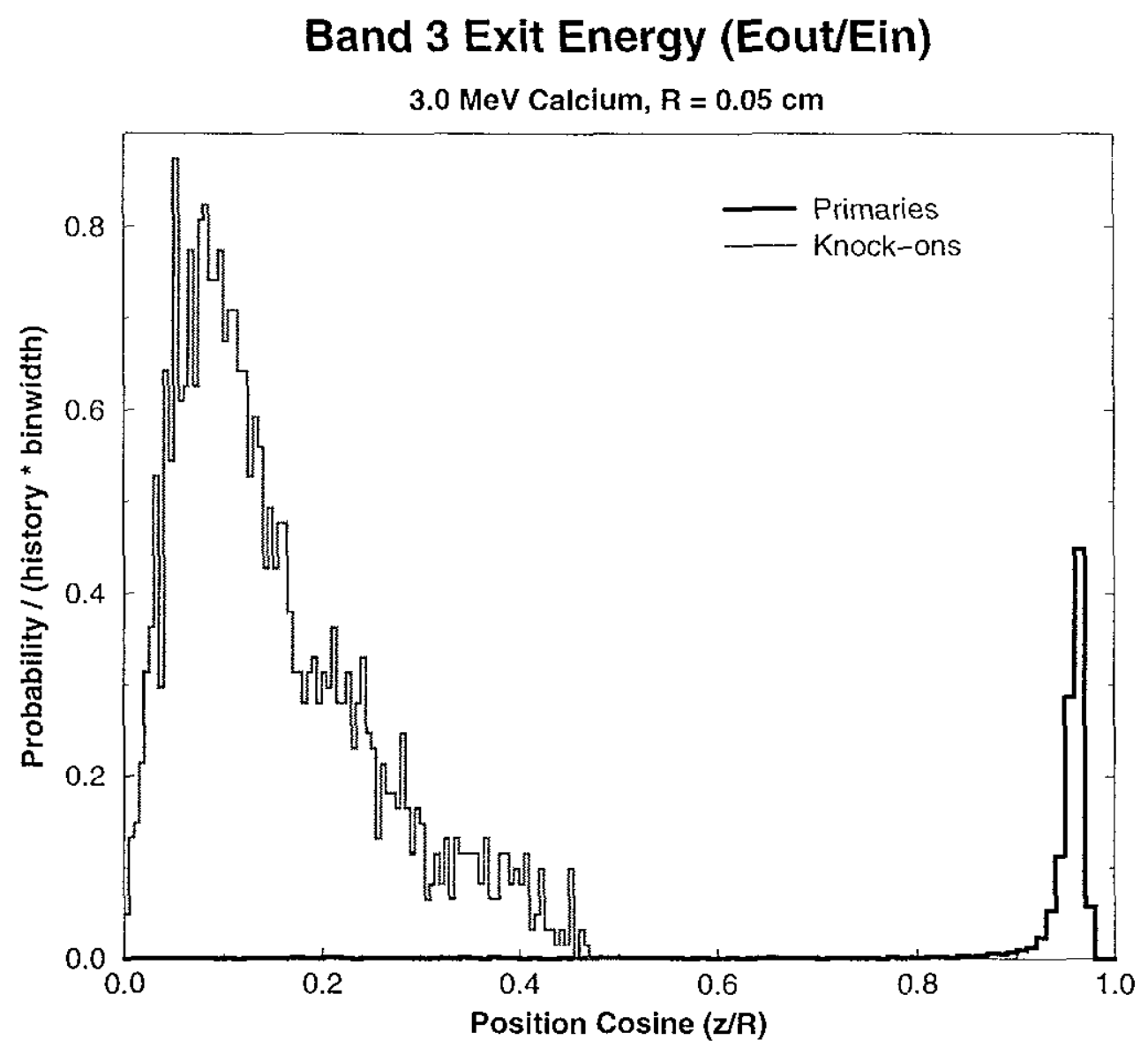

Figure 50: Exiting energy for primary electrons and escaping knock-ons from Band 3 of a $3 \mathrm{MeV}$ calcium kugel of radius $0.05 \mathrm{~cm}$. 


\section{Band 4 Exit Energy (Eout/Ein)}

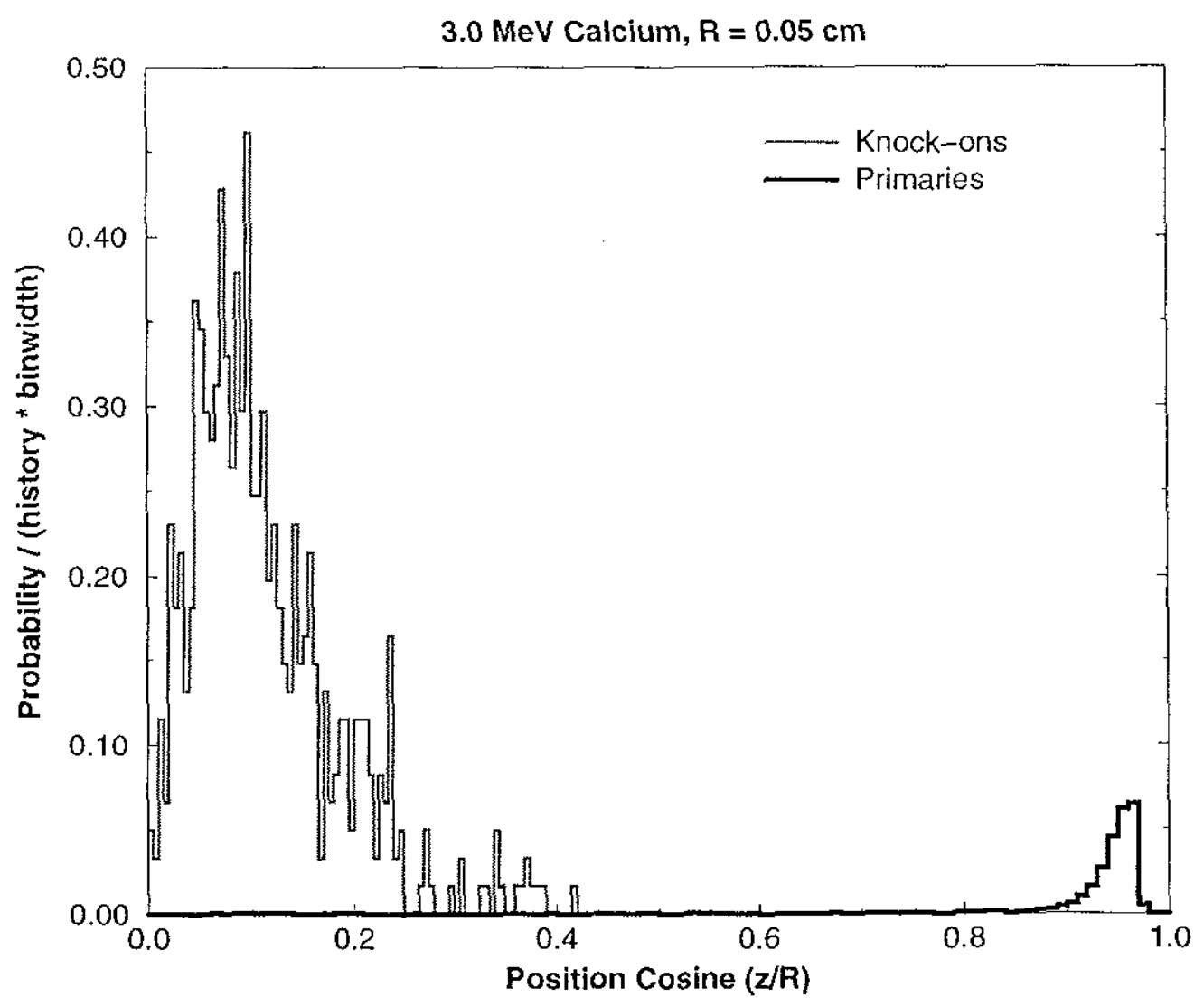

Figure 51: Exiting energy for primary electrons and escaping knock-ons from Band 4 of a $3 \mathrm{MeV}$ calcium kugel of radius $0.05 \mathrm{~cm}$. 


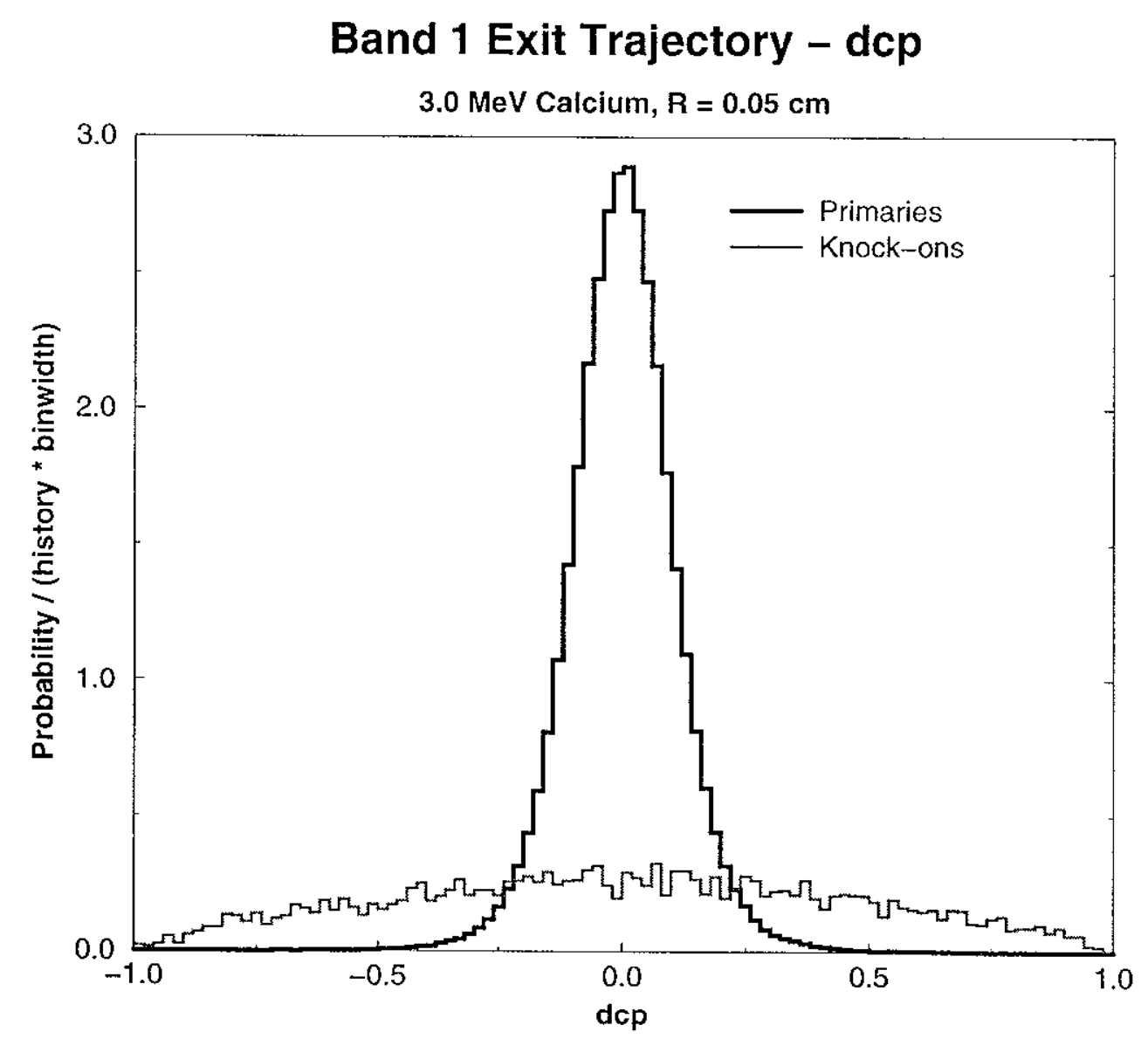

Figure 52: Exiting trajectory $(d c p)$ for primary electrons and escaping knock-ons from Band 1 of a $3 \mathrm{MeV}$ calcium kugel of radius $0.05 \mathrm{~cm}$. See Section 3.3 .3 for an explanation of the trajectory tallies. 


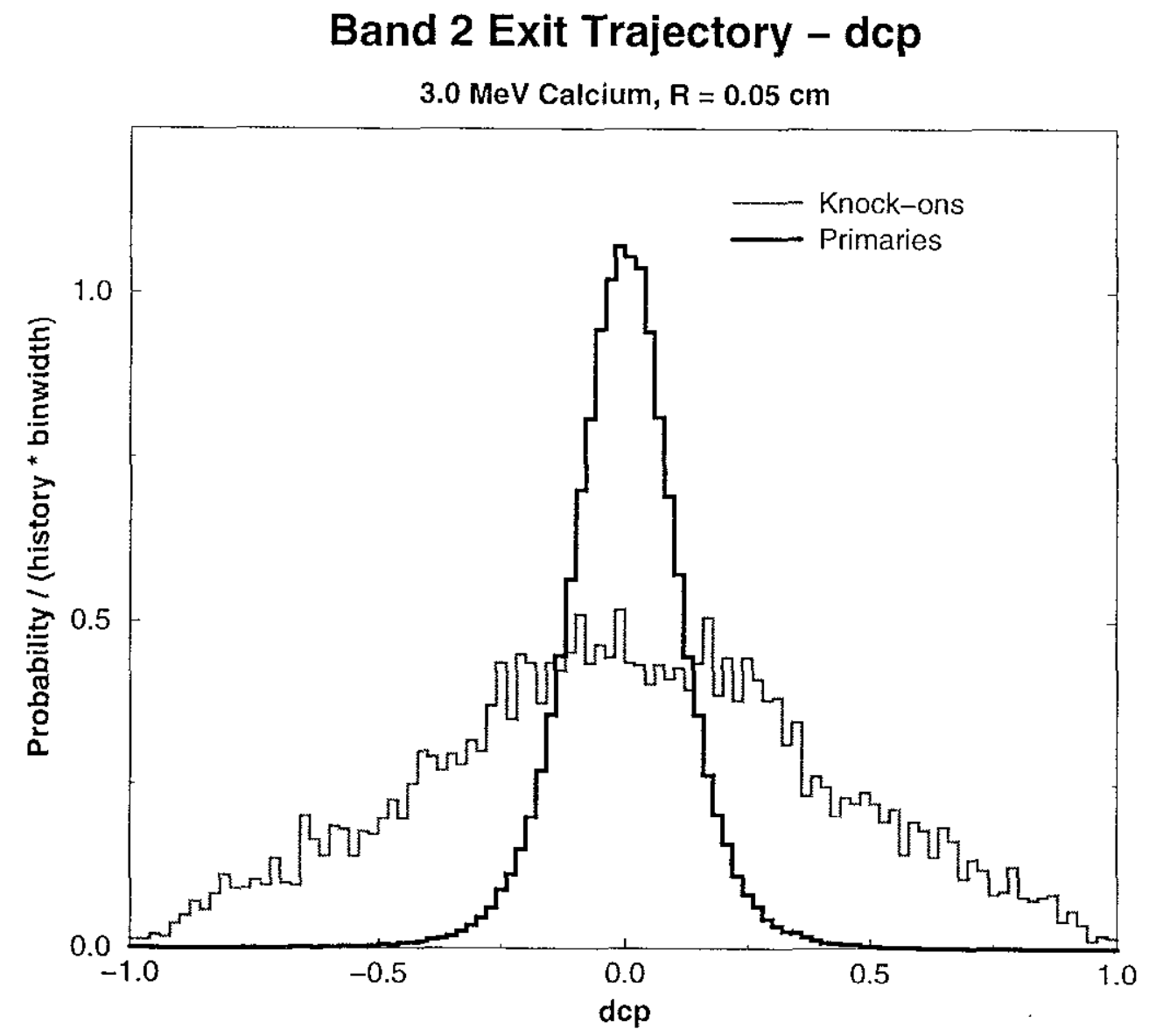

Figure 53: Exiting trajectory $(d c p)$. for primary electrons and escaping knock-ons from Band 2 of a $3 \mathrm{MeV}$ calcium kugel of radius $0.05 \mathrm{~cm}$. See Section 3.3 .3 for an explanation of the trajectory tallies. 


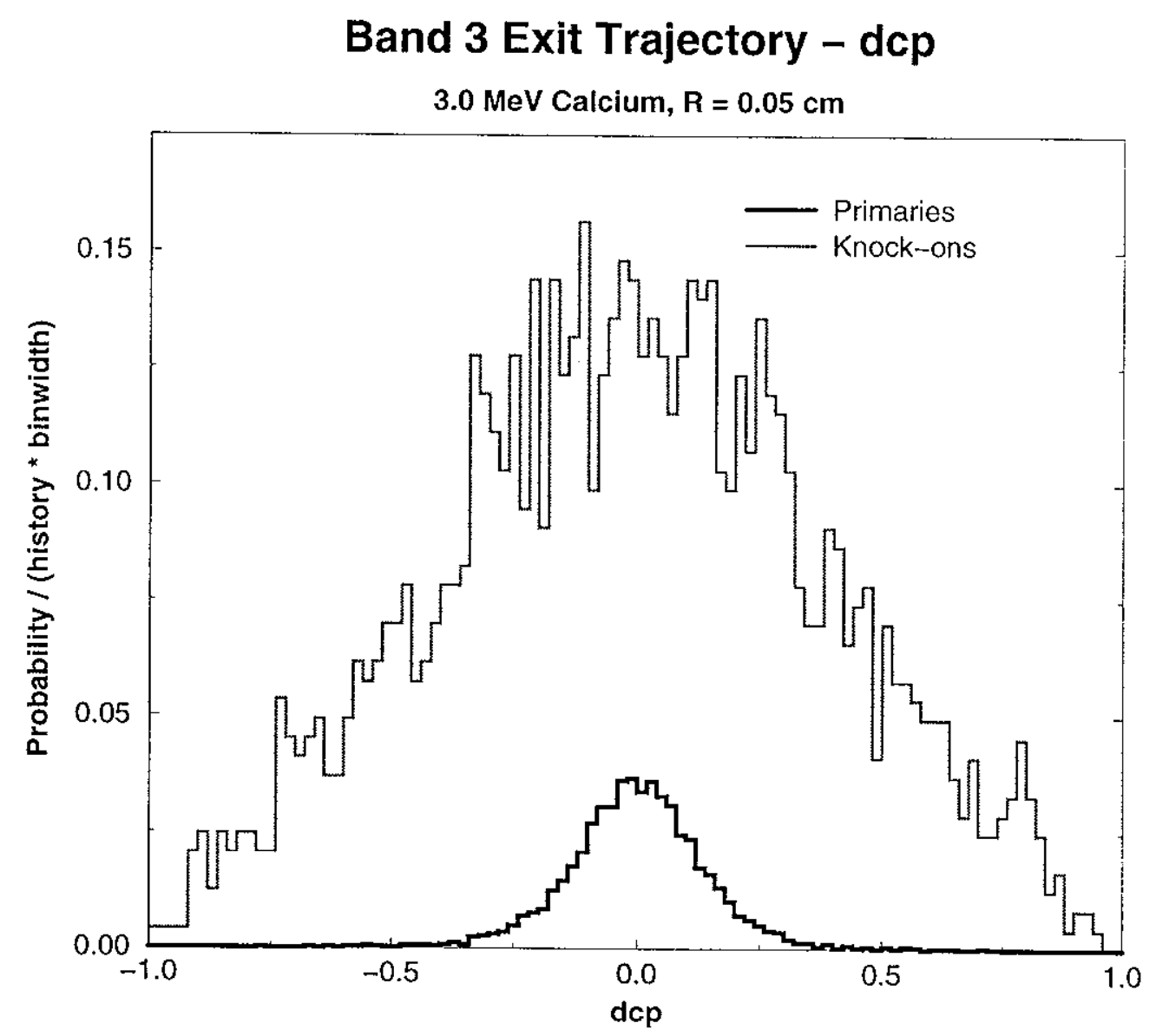

Figure 54: Exiting trajectory $(d c p)$ for primary electrons and escaping knock-ons from Band 3 of a $3 \mathrm{MeV}$ calcium kugel of radius $0.05 \mathrm{~cm}$. See Section 3.3 .3 for an explanation of the trajectory tallies. 


\section{Band 4 Exit Trajectory - dcp}

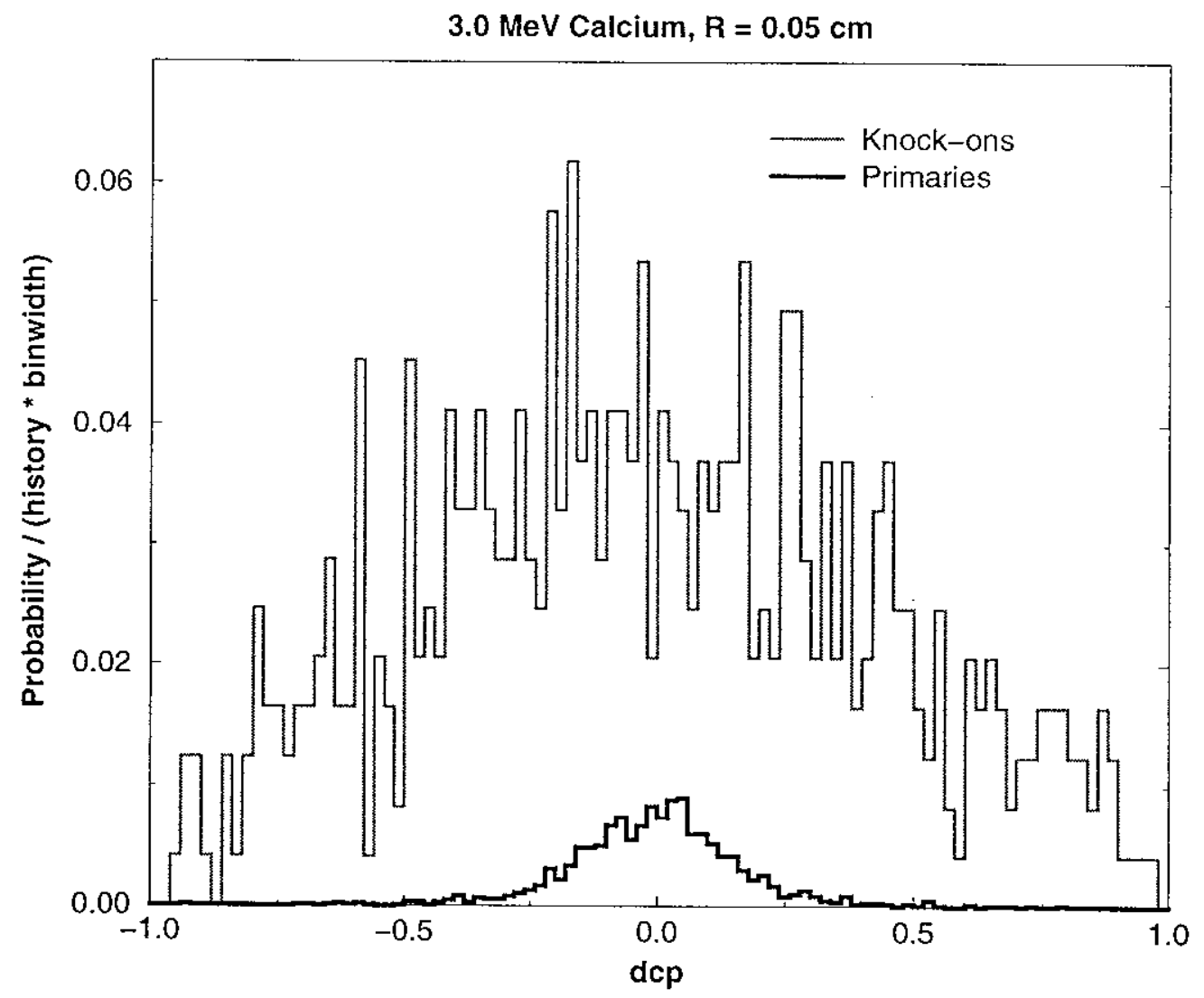

Figure 55: Exiting trajectory $(d c p)$ for primary electrons and escaping knock-ons from Band 4 of a $3 \mathrm{MeV}$ calcium kugel of radius $0.05 \mathrm{~cm}$. See Section 3.3 .3 for an explanation of the trajectory tallies. 


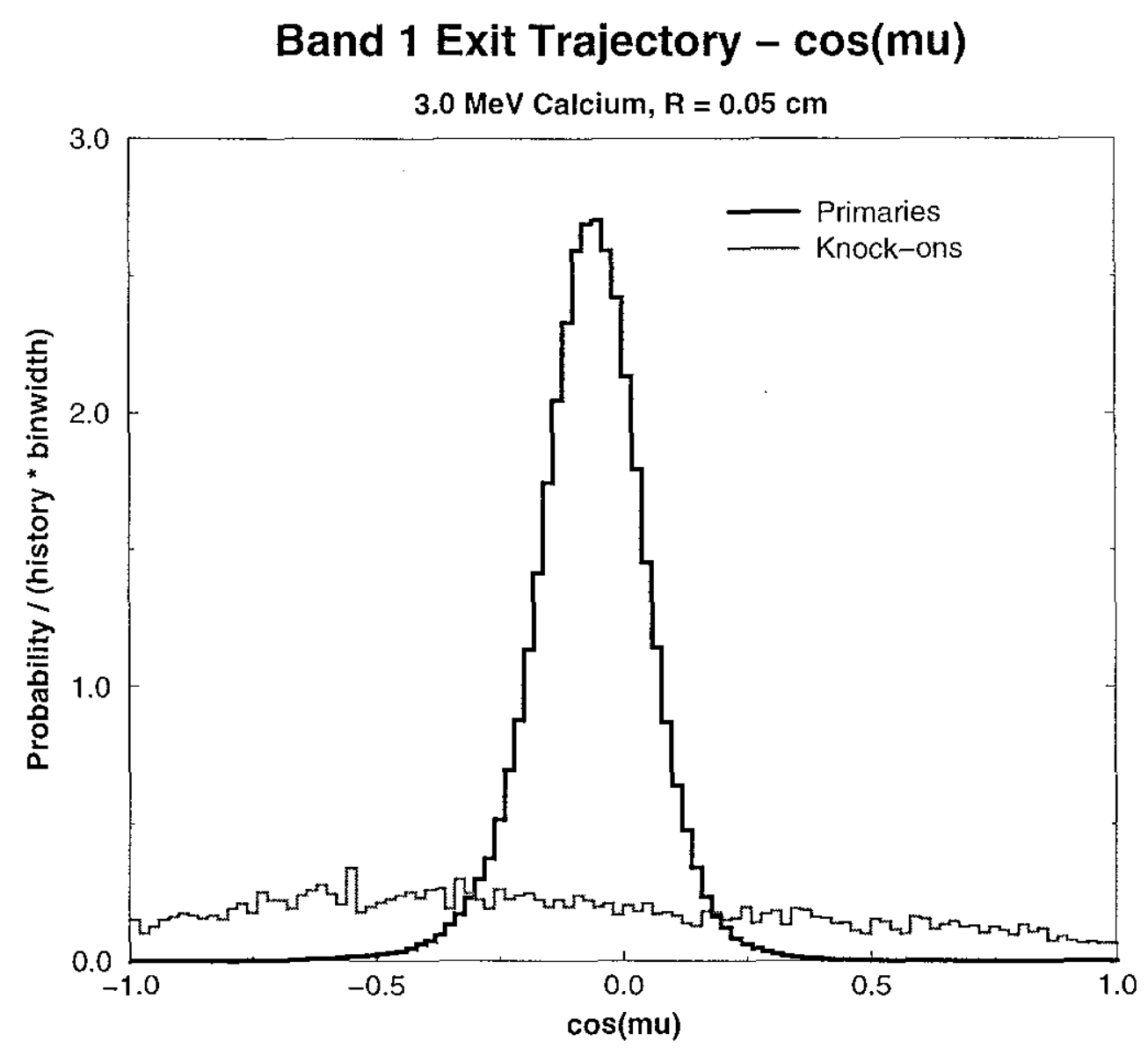

Figure 56: Exiting trajectory $(\cos \mu)$ for primary electrons and escaping knock-ons from Band 1 of a $3 \mathrm{MeV}$ calcium kugel of radius $0.05 \mathrm{~cm}$. See Section 3.3 .3 for an explanation of the trajectory tallies. 


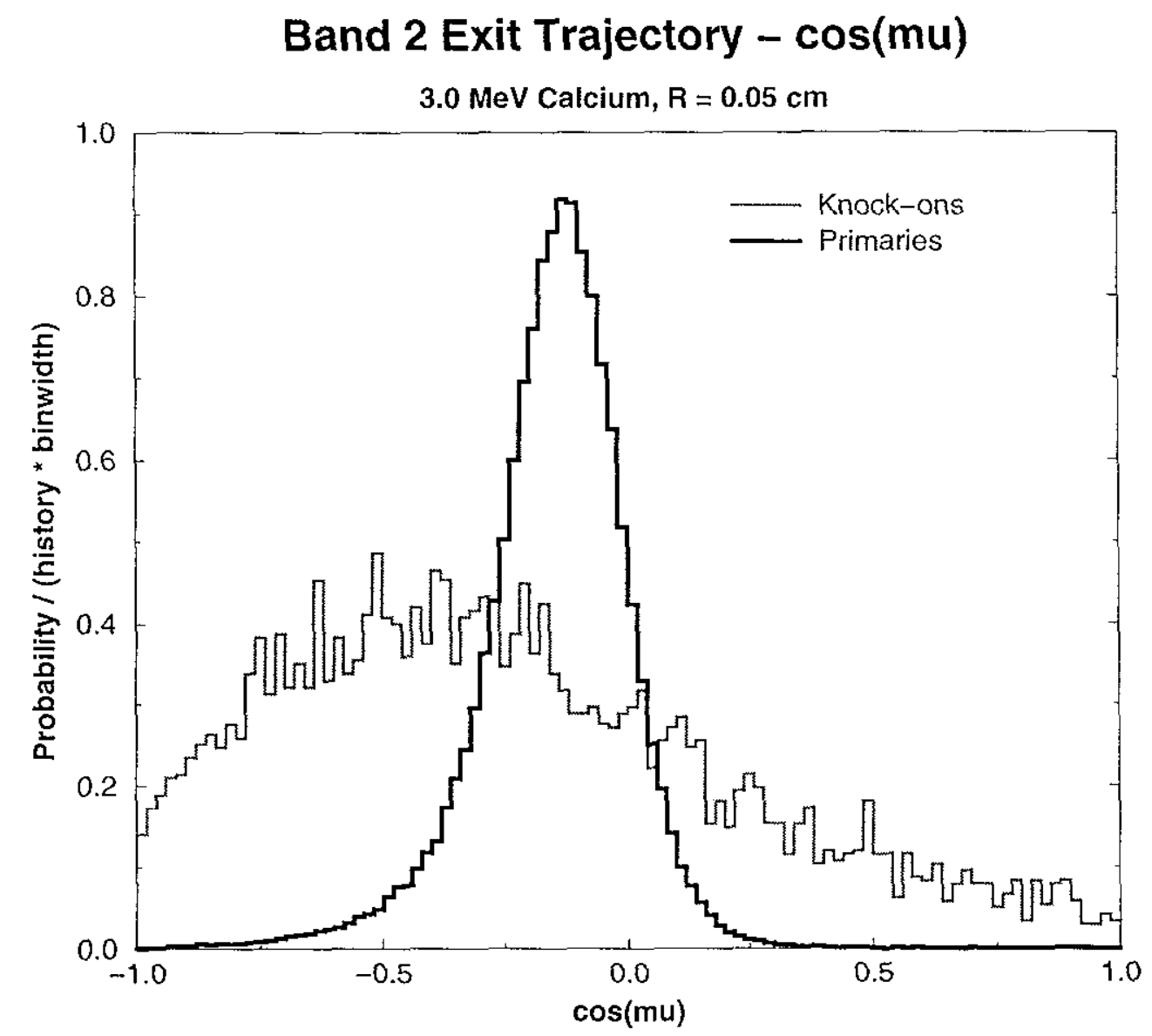

Figure 57: Exiting trajectory $(\cos \mu)$ for primary electrons and escaping knock-ons from Band 2 of a $3 \mathrm{MeV}$ calcium kugel of radius $0.05 \mathrm{~cm}$. See Section 3.3 .3 for an explanation of the trajectory tallies. 


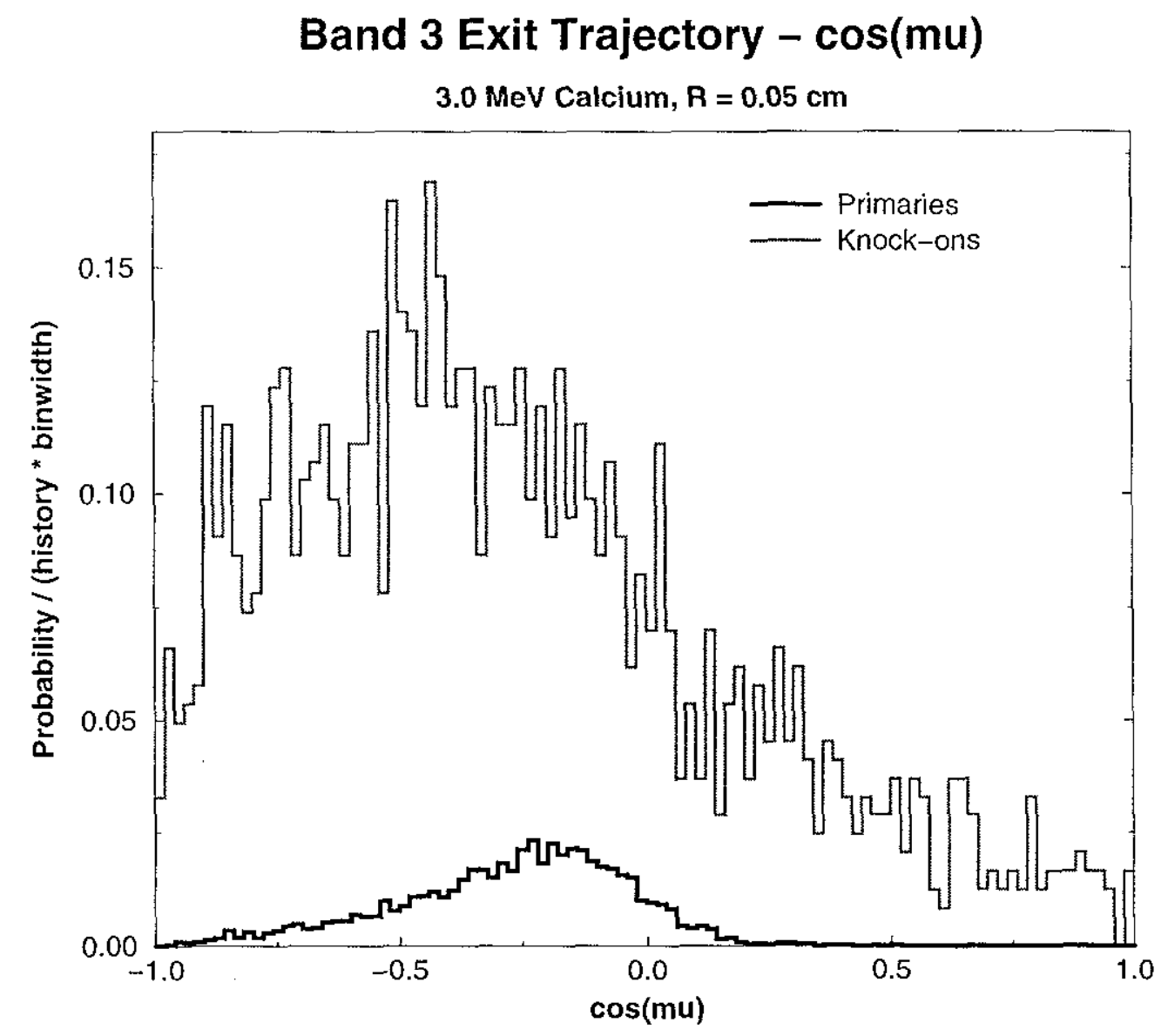

Figure 58: Exiting trajectory $(\cos \mu)$ for primary electrons and escaping knock-ons from Band 3 of a $3 \mathrm{MeV}$ calcium kugel of radius $0.05 \mathrm{~cm}$. See Section 3.3 .3 for an explanation of the trajectory tallies. 


\section{Band 4 Exit Trajectory - $\cos (\mathrm{mu})$}

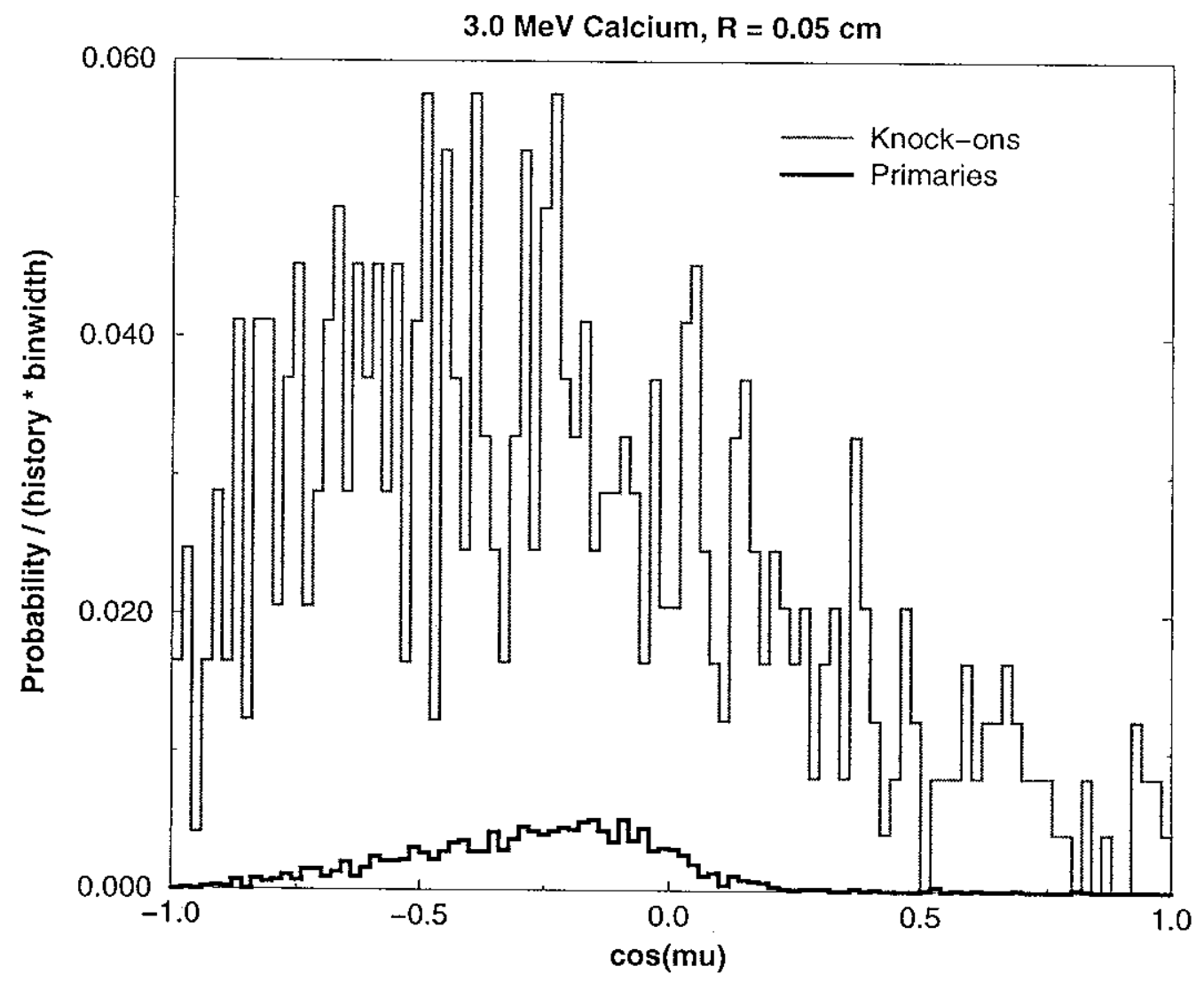

Figure 59: Exiting trajectory $(\cos \mu)$ for primary electrons and escaping knock-ons from Band 4 of a $3 \mathrm{MeV}$ calcium kugel of radius $0.05 \mathrm{~cm}$. See Section 3.3 .3 for an explanation of the trajectory tallies. 


\section{Average Number of Knock-ons Escaping}

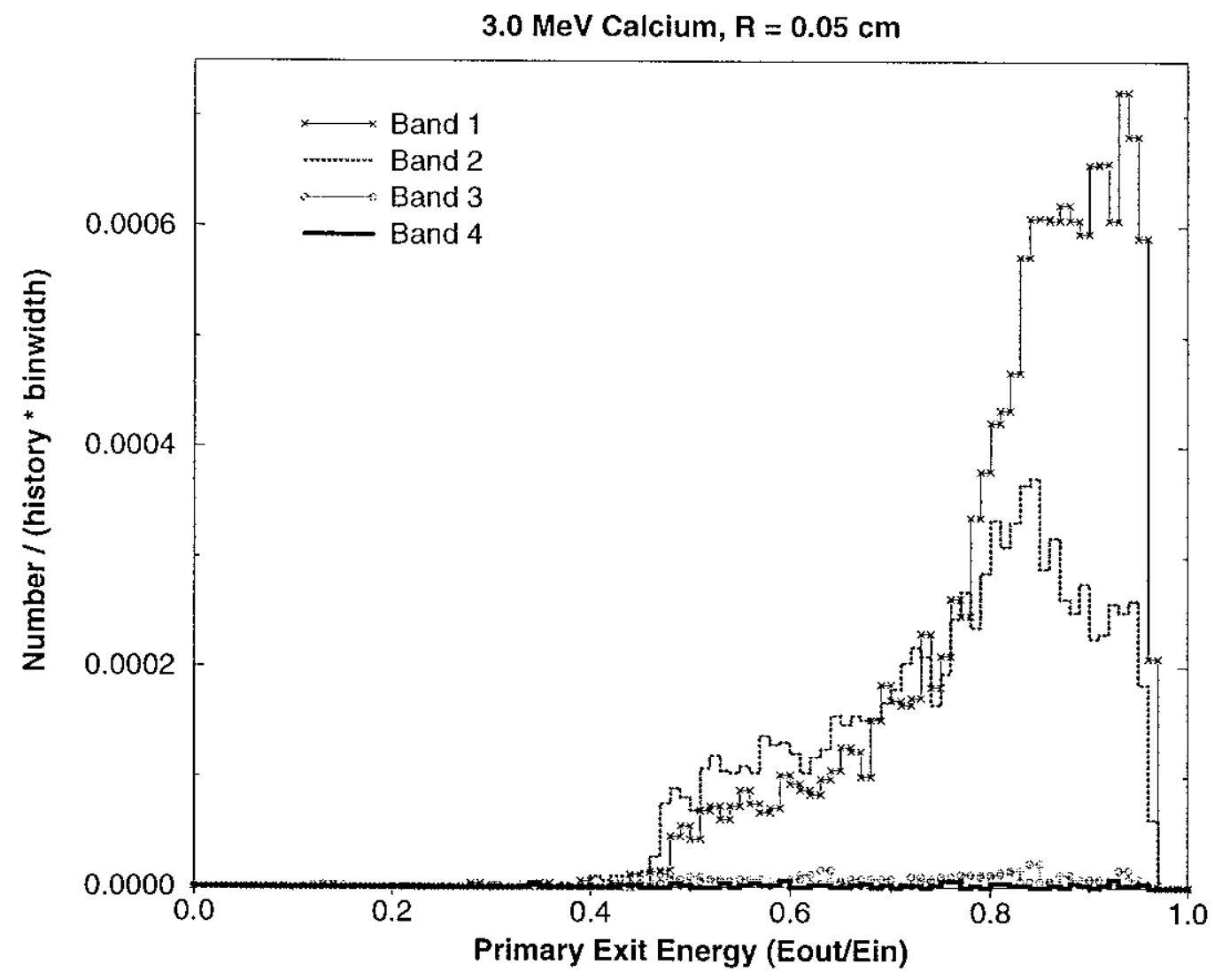

Figure 60: Average number of escaping knock-ons from a $3 \mathrm{MeV}$ calcium kugel of radius $0.05 \mathrm{~cm}$. See Section 3.4 .3 for an explanation of these tallies. 


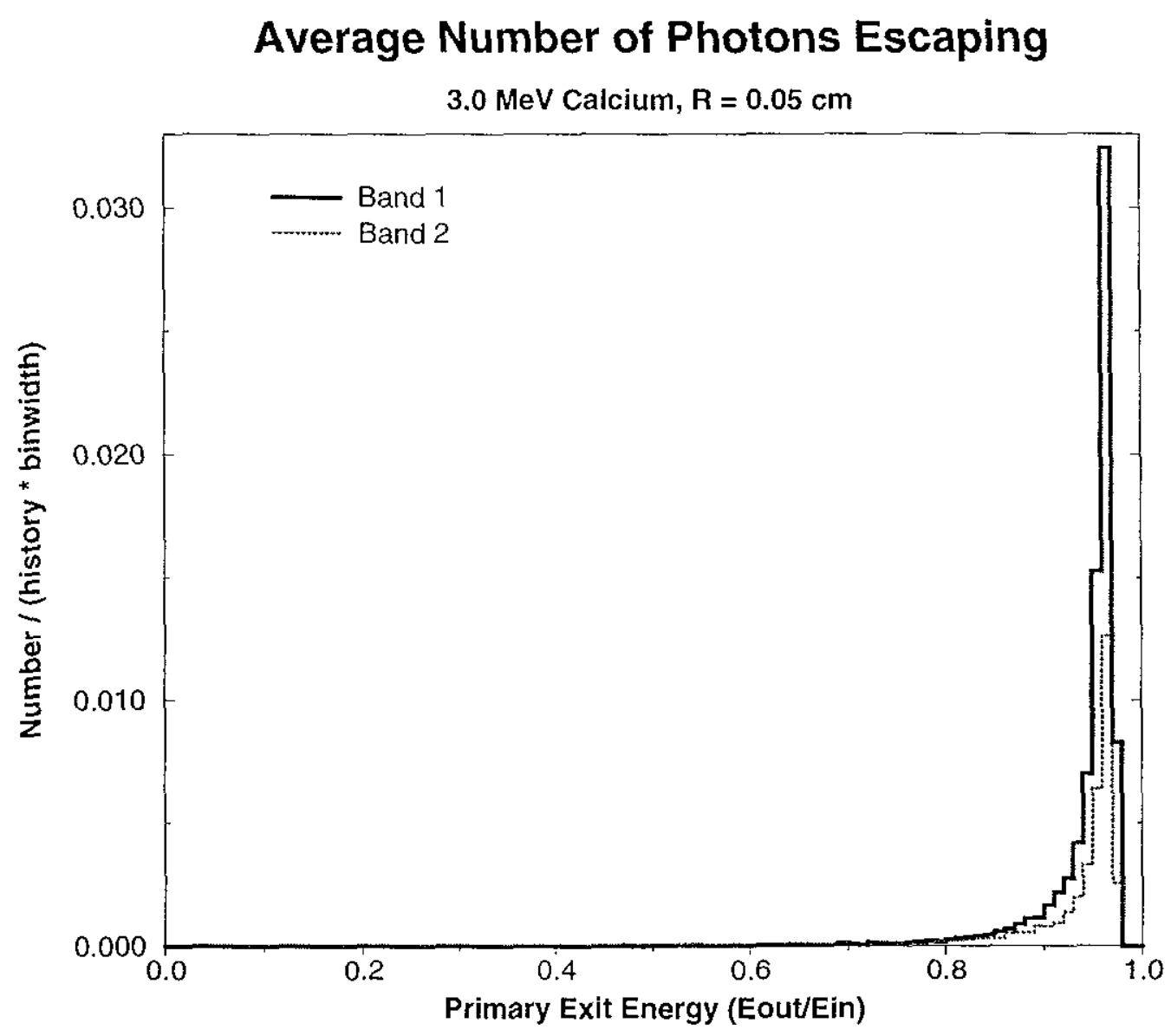

Figure 61: Average number of escaping bremsstrahlung photons from Bands 1 and 2 of a $3 \mathrm{MeV}$ calcium kugel of radius $0.05 \mathrm{~cm}$. See Section 3.4 .4 for an explaination of these tallies. 


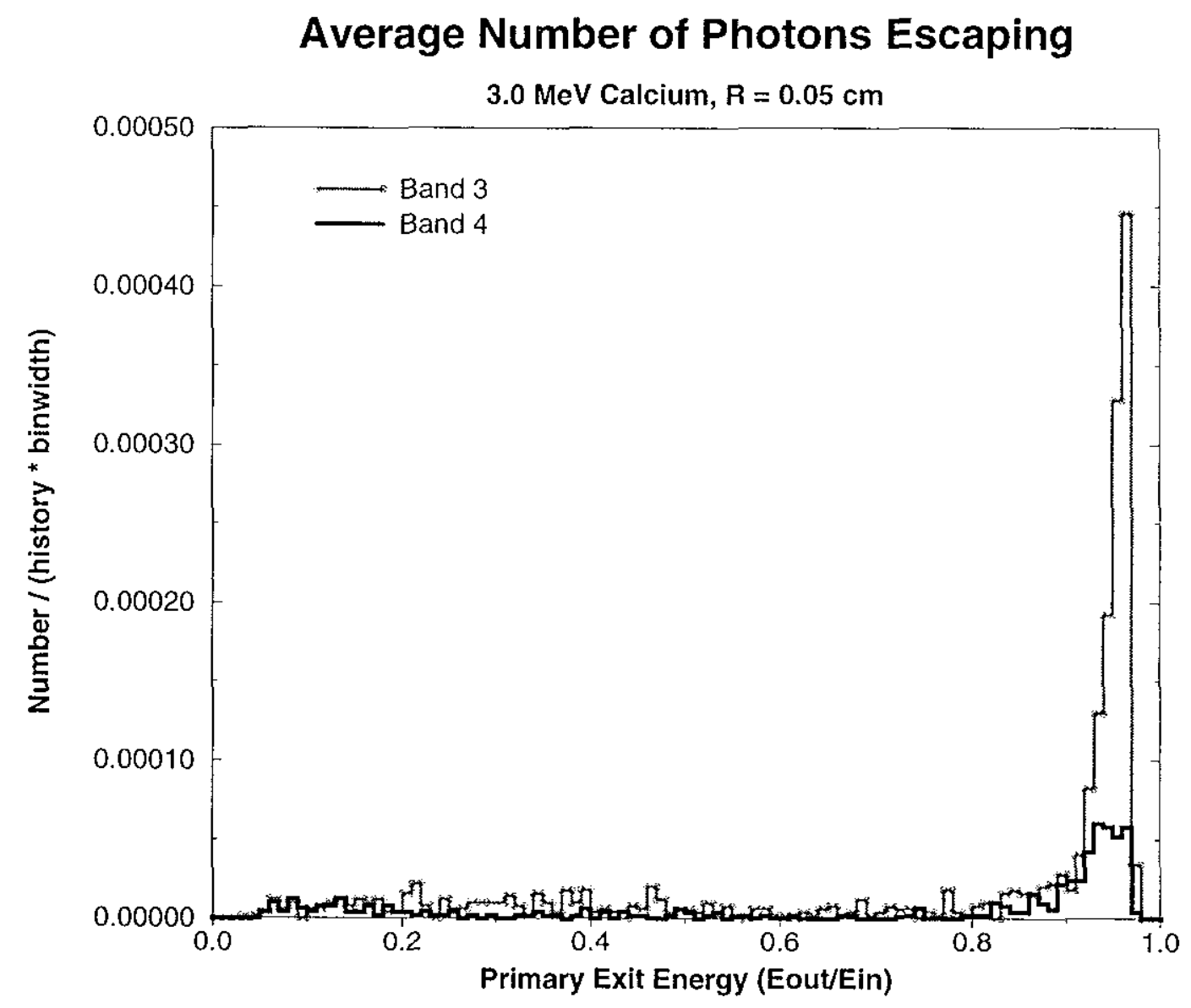

Figure 62: Average number of escaping bremsstrahlung photons from Bands 3 and 4 of a $3 \mathrm{MeV}$ calcium kugel of radius $0.05 \mathrm{~cm}$. See Section 3.4 .4 for an explaination of these tallies. 


\section{Bibliography}

[1] P. Almond, A. Wright, And M. Boone, High energy electron dose perturbations in regions of tissue heterogeneity: Part II: Physical models of tissue heterogeneities, Radiology, 88 (1967), pp. 1146-1153.

[2] P. Andreo, Monte Carlo techniques in medical radiation physics, Phys. Med. Biol., 36 (1991), pp. 861-920.

[3] C. Ballinger, The Response History Method for Electron Transport, PhD thesis, University of Michigan, Lawrence Livermore National Laboratory, 1991.

[4] C. Ballinger, D. Cullen, S. Perkins, J. Rathkopf, W. Martin, and S. Wilderman, Single-scatter Monte Carlo compared to condensed history results for low energy electrons, Nuclear Instruments and Methods in Physics Research B, (1992), pp. 19-27.

[5] M. BERGER, Monte Carlo calculation of the penetration and diffusion of fast charged particles, Methods in Computational Physics, 1 (1963), pp. 135-215.

[6] M. Berger And S. Seltzer, Bremsstrahlung and photoneutrons from thick tungsten and tantalum targets, Physical Review, C (1970), pp. 621-631.

[7] M. BERGER AND R. WANG, Multiple-scattering angular deflections and energy loss straggling, in Monte Carlo Transport of Electrons and Photons, T. M. Jenkins, W. Nelson, and A. Rindi, eds., New York and London, 1988, Plenum Press, pp. 21-55.

[8] H. A. Bethe, Moliere's theory of multiple scattering, Physical Review, 89 (1953), pp. 1256-1266. 
[9] A. Bielajew, Plural and multiple small-angle scattering from a screened Rutherford cross section, Nuclear Instruments and Methods, B86 (1994), pp. 257-269.

[10] O. BlunCK AND K. WeSTPHAL, Zum energieverlust energiereicher elektronen in dunnen schichten, Z Phys., 130 (1951), p. 641.

[11] T. E. Воотн, A sample problem for variance reduction in MCNP, Tech. Rep. LA-10363-MS, Los Alamos National Laboratory, 1985.

[12] T. E. Booth AND S. S. Pederson, Unbiased combinations of nonanalog Monte Carlo techniques and fair games, Nuclear Science and Engineering, 110 (1992), pp. 254-261.

[13] J. Briemeister, MCNP4 - a general Monte Carlo code for neutron, photon, and electron transport, Tech. Rep. LA-7936-M Rev. 4, Los Alamos National Laboratory, 1991.

[14] W. Chandler, personal communication, june 1994.

[15] H. COLBERT, SANDYL: A computer program for calculating combined photon-electron transport in complex systems, Tech. Rep. SLL-74-0012, Sandia National Laboratory - Livermore, CA, 1974.

[16] L. J. Cox, A. Schach von Wittenau, P. M. Bergstrom, R. Mohan, B. LibBY, Q. WU, AND D. Lovelock, Treatment of patient-dependent beam modifiers in photon treatments by the Monte Carlo dose calculation code Peregrine, in Proceedings of the XIIth International Conference on the Use of Computers in Radiation Therapy, Madison, WI, 1997, Medical Physics Publishing.

[17] D. Cullen And S. Perkins, The Livermore bremsstrahlung database, Tech. Rep. UCID-21627, Lawrence Livermore National Laboratory, 1989. 
[18] J. Cygler, J. Battista, J. Scrimger, E. Mah, and J. Antolak, Electron dose distributions in experimental phantoms: a comparision with $2 D$ pencil beam calculations, Phys Med Biol, 32 (1987), pp. 1073-86.

[19] E. Darlington, Backscattering of 10-100 kev electrons from thick targets, J. Phys. D Appl. Phys, 8 (1975), pp. 85-93.

[20] J. J. Demarco, T. Solberg, and J. B. Smathers, A CT-based Monte Carlo dosimetry tool for radiotherapy treatment planning and analysis, Medical Physics, 25 (1998), pp. 1-11.

[21] J. Du, Response Kernel Density Estimation Monte Carlo Method for Electron Transport, PhD thesis, University of Michigan, Amn Arbor, Michigan, 1997.

[22] A. L. Edwards, J. A. Rathkopf, and R. K. Smidt, Extending the Alias Monte Carlo sampling method to general distributions, in Proceedings of the International Conference on Mathematics and Computations, and Reactor Physics, La Grange Park, IL, 1991, American Nuclear Society, Inc.

[23] L. Eyges, Multiple scattering with energy loss, Phys. Rev., 74 (1948), pp. 1534-5.

[24] S. Goudsmit and J. L. Saunderson, Multiple scattering of electrons, Physical Review, 57 (1940), pp. 24-29.

$[25]$ - Multiple scattering of electrons II, Physical Review, 58 (1940), pp. 3642.

[26] J. HALBLEIB, Structure and operations of the ITS code system, in Monte Carlo Transport of Electrons and Photons, T. M. Jenkins, W. Nelson, and A. Rindi, eds., New York and London, 1988, Plenum Press. 
[27] O. K. Harding, K. A. Roberts, D. J. Moulin, and R. D. Rogus, Head phantoms for neutron capture therapy, Medical Physics, 22 (1995), p. 579.

[28] O. K. Harding, J. C. Yanch, J. R. Choi, G. R. Solares, R. Rogus, D. J. Moulin, L. S. Johnson, I. Olmez, S. Wirdzek, J. A. Bernard, R. G. Zamenhof, C. I. Nwanguma, D. E. Wazer, S. Saris, H. Madoc-Jones, C. B. Sledge, and S. Shortkroff, Boron neutron capture therapy and radiation synovectomy research at the Massachusetts Institute of Technology research reactor, Nuclear Science and Engineering, 110 (1992), pp. 330-348.

[29] O. K. Harding, R. G. Zamenhof, G. R. Solares, J. C. Yanch, D. E. Wazer, J. Chabeuf, S. C. Yam, J. A. Bernard, G. Cano, T. DiPetrillo, And H. Madoc-Jones, Preparations for phase I clinical trials for boron neutron capture therapy at the MIT reactor and the New England medical center, Radiation Oncology Investigations, 2 (1984), pp. 109118.

[30] C. Hartmann Siantar, P. M. Bergstrom, W. Chandler, L. Chase, L. J. Cox, T. Daly, D. Garrett, S. M. Hornstein, R. K. House, E. Moses, R. W. Patterson, J. Rathkopf, and A. E. Schach von WitTenau, Lawrence Livermore National Laboratory's Peregrine project, in Proceedings of the XIIth International Conference on the Use of Computers in Radiation Therapy, Madison, WI, 1997, Medical Physics Publishing.

[31] R. J. HEDGER, An evaluation of the Monte Carlo radiation transport code MCNP4A for the purpose of radiotherapy simulation, Master's thesis, University of Birmingham, 1993.

[32] K. Hogstrom, M. Mills, and P. Almond, Electron beam dose calculations, Phys. Med. Biol., 26 (1981), pp. 445-459. 
[33] D. HunERSon, MCNP in radiotherpy and an investigation of dosimetry in irregular fields and scatter effects at field edges, Master's thesis, University of Birmingham, 1993.

[34] H. KANTER, Zur ruckstreuung von elektronen im energiebereich von 10 bis 100 kev, Annalen der Physik, 20 (1957), pp. 144-166.

[35] L. LANDAU, On the energy loss of fast particles by ionization, Journal of Physics USSR, 8 (1944), pp. 201-5.

[36] J. LAUGHLin, High energy electron treatment planning for inhomogeneities, British Journal of Radiography, 38 (1965), pp. 143-147.

[37] H. B. Liu, R. M. Brugger, B. H. Laster, D. D. Greenberg, C. R. Gordon, And L. S. Warkentien, Physical and biological doses produced from neutron capture in a $235 U$ foil, Medical Physics, 22 (1995), p. 591.

[38] G. Lockwood, L. Ruggles, G. Miller, and J. Halbleib, Calorimetric measurement of electron energy deposition in extended media - theory vs experiment, Tech. Rep. SAND79-0414, Sandia National Labortory, 1980.

[39] T. MACKIE, Applications of the Monte Carlo method in radiotherapy, in The Dosimetry of Ionizing Radiation, Vol. III, F. Attix, K. Kase, and B. Bjarngard, eds., Academic Press, Inc., 1990, pp. 541-620.

[40] T. Mackie and J. Battista, A Macroscopic Monte Carlo method for electron beam dose calculations: A proposal, in Eighth International Conference on the Use of Computers in Radiation Therapy, Toronto, 1984, IEEE.

[41] W. Nelson, H. Hirayama, and D. Rogers, The EGS4 code system, Stanford Linear Accelerator Center Report 265, (1985). 
[42] W. R. Nelson And D. W. O. Rogers, Structure and operation of the EGS4 code system, in Monte Carlo Transport of Electrons and Photons, T. M. Jenkins, W. Nelson, and A. Rindi, eds., New York and London, 1988, Plenum Press.

[43] G. NEubert and S. RoGaschewski, Backscattering coefficient measurements of 15 to 60 kev electrons for solids at various angles of incidence, Phys. Stat. Sol. (a), 59 (1980), pp. 35-41.

[44] H. Neuenschwander and E. Born, A Macro Monte Carlo method for electron beam dose calculations, Phys. Med. Biol., 37 (1992), pp. 107-125.

[45] H. Neuenschwander, T. Mackie, and P. Reckwerdt, $M M C$ - a high performance Monte Carlo code for electron beam dose calculations, Phys. Med. Biol, 40 (1995), pp. 543--574.

[46] H. Neuenschwander, W. Volken, C. Cris, R. Mini, and P. Schwab, Fast Monte Carlo algorithms for electron beam treatment planning, in Proceedings of the XIIth International Conference on the Use of Computers in Radiation Therapy, Madison, WI, 1997, Medical Physics Publishing.

[47] I. C. on Radiation Units and Measurements, Stopping powers for electrons and positrons, october 1984.

[48] S. Perkins and D. Cullen, The Livermore electron impact ionization database, Tech. Rep. UCID-21628, Lawrence Livermore National Laboratory, 1989.

[49] —, The Livermore electron elastic scattering database, Tech. Rep. UCRLID-103170, Lawrence Livermore National Laboratory, 1990. 
[50] R. Rogus, O. K. Harding, and J. C. Yanch, Mixed field dosimetry of epithermal neutron beams for boron neutron capture therapy at the MITR - II research reactor, Medical Physics, 21 (1993), pp. 1611-1625.

[51] A. Schach von Wittenau, L. J. Cox, P. M. Bergstrom, R. Mohan, B. LibBy, Q. Wu, AND D. LovelOCK, Treatment of patient-dependent beam modifiers in photon treatments by the Monte Carlo dose calculation code Peregrine, in Proceedings of the XIIth International Conference on the Use of Computers in Radiation Therapy, Madison, WI, 1997, Medical Physics Publishing.

[52] L. SCHIFF, Energy-angle distribution of thin target bremsstrahlung, Physical Review, 83 (1951), pp. 252-3.

[53] W. SCOTT, The theory of small-angle multiple scattering of fast charged particles, Reviews of Modern Physics, 35 (1963), pp. 231-313.

[54] S. Seltzer and M. Berger, Atomic Data and Nuclear Data Tables, 35 (1985), p. 345 .

[55] K. Shortt, C. Ross, A. Bielajew, ANd . D. Rogers, Electron beam dose distributions near standard inhomogeneities, Phys Med Biol, 31 (1986), pp. 235-249.

[56] M. Svatos, C. Ballinger, H. Neuenschwander, T. Mackie, W. Chandler, C. Hartmann Siantar, J. Rathkopf, and P. ReckwERDT, Electron transport in radiotherapy using Local-to-Global Monte Carlo, in Proceedings of the International Conference on Mathematics and Computations, Reactor Physics, and Environmental Analyses, La Grange Park, IL, 1995, American Nuclear Society, Inc., pp. 866-875. 
[57] Y. TSAI, Pair production and bremsstrahlung of charged leptons, Rev. Mod. Physics, 46 (1974), p. 815.

[58] H. Tseng and R. Pratt, Phys. Rev. Let, 33 (1974), p. 516.

[59] W. Wan, Response Matrix Monte Carlo, PhD thesis, University of Michigan, Ann Arbor, Michigan, 1988. 